University of Louisville

ThinkIR: The University of Louisville's Institutional Repository

Electronic Theses and Dissertations

$12-2014$

\title{
Shape/image registration for medical imaging : novel algorithms and applications.
}

Ahmed Magdy Shalaby 1982-

University of Louisville

Follow this and additional works at: https://ir.library.louisville.edu/etd

Part of the Biomedical Commons, and the Radiology Commons

\section{Recommended Citation}

Shalaby, Ahmed Magdy 1982-, "Shape/image registration for medical imaging : novel algorithms and applications." (2014). Electronic Theses and Dissertations. Paper 1768.

https://doi.org/10.18297/etd/1768

This Doctoral Dissertation is brought to you for free and open access by ThinkIR: The University of Louisville's Institutional Repository. It has been accepted for inclusion in Electronic Theses and Dissertations by an authorized administrator of ThinkIR: The University of Louisville's Institutional Repository. This title appears here courtesy of the author, who has retained all other copyrights. For more information, please contact thinkir@louisville.edu. 


\title{
SHAPE/IMAGE REGISTRATION FOR MEDICAL IMAGING: NOVEL ALGORITHMS AND APPLICATIONS
}

\author{
By \\ Ahmed Magdy Shalaby \\ B.Sc. in Electrical Engineering, Alexandria University, 2003 \\ M.Sc. in Electrical Engineering, Alexandria University, 2009
}

\author{
A Dissertation \\ Submitted to the Faculty of the \\ J. B. Speed School of Engineering of the University of Louisville \\ in Partial Fulfillment of the Requirements \\ for the Degree of
}

\author{
Doctor of Philosophy \\ Department of Electrical and Computer Engineering \\ University of Louisville \\ Louisville, Kentucky
}

December 2014 



\title{
SHAPE/IMAGE REGISTRATION FOR MEDICAL IMAGING: NOVEL ALGORITHMS AND APPLICATIONS
}

\author{
By \\ Ahmed Magdy Shalaby \\ B.Sc. in Electrical Engineering, Alexandria University, 2003 \\ M.Sc. in Electrical Engineering, Alexandria University, 2009
}

A Dissertation Approved on

November 20, 2014

by the following Dissertation Committee:

Aly A. Farag, Ph.D., Dissertation Director

James Graham, Ph.D.

Tamer Inanc, Ph.D.

R. Todd Hockenbury, M.D.

Chin Ng, Ph.D. 


\section{DEDICATION}

I would like to dedicate this dissertation to my country "EGYPT" which gave me invaluable educational opportunities, my great mother "GALILA" and my beloved wife "REHAM" for their love and patience. 


\section{ACKNOWLEDGEMENTS}

First of all, I would like to thank God the merciful, the compassionate for all the blessings and for granting me the opportunity to accomplish my $\mathrm{PhD}$ degree successfully.

I would like to express my deepest gratitude to my advisor Dr. Aly A. Farag for his guidance, support, and encouragement to pursue a $\mathrm{Ph} . \mathrm{D}$. degree in the computer vision and medical imaging field.

I would like to acknowledge the other members of my Ph.D. committee for spending time and effort in reading and reviewing my work Dr. James H. Graham, Dr. R. Todd Hockenbury, Dr. Tamer Inanc, and Dr.Chin Ng.

Also, I would like to thank to Mr. Mike Miller, and all CVIP Lab members for their friendship and assistance. 


\title{
ABSTRACT \\ SHAPE/IMAGE REGISTRATION FOR MEDICAL IMAGING: NOVEL ALGORITHMS AND APPLICATIONS
}

\author{
Ahmed M. Shalaby
}

Novmber 20, 2014

This dissertation looks at two different categories of the registration approaches: Shape registration, and Image registration. It also considers the applications of these approaches into the medical imaging field.

Shape registration is an important problem in computer vision, computer graphics and medical imaging. It has been handled in different manners in many applications like shapebased segmentation, shape recognition, and tracking. Image registration is the process of overlaying two or more images of the same scene taken at different times, from different viewpoints, and/or by different sensors. Many image processing applications like remote sensing, fusion of medical images, and computer-aided surgery need image registration.

This study deals with two different applications in the field of medical image analysis. The first one is related to shape-based segmentation of the human vertebral bodies (VBs). The vertebra consists of the VB, spinous, and other anatomical regions. Spinous pedicles, and ribs should not be included in the bone mineral density (BMD) measurements. The VB segmentation is not an easy task since the ribs have similar gray level information. 
This dissertation investigates two different segmentation approaches. Both of them are obeying the variational shape-based segmentation frameworks. The first approach deals with two dimensional (2D) case. This segmentation approach starts with obtaining the initial segmentation using the intensity/spatial interaction models. Then, shape model is registered to the image domain. Finally, the optimal segmentation is obtained using the optimization of an energy functional which integrating the shape model with the intensity information. The second one is a 3D simultaneous segmentation and registration approach. The information of the intensity is handled by embedding a Willmore flow into the level set segmentation framework. Then the shape variations are estimated using a new distance probabilistic model. The experimental results show that the segmentation accuracy of the framework are much higher than other alternatives. Applications on BMD measurements of vertebral body are given to illustrate the accuracy of the proposed segmentation approach.

The second application is related to the field of computer-aided surgery, specifically on ankle fusion surgery. The long-term goal of this work is to apply this technique to ankle fusion surgery to determine the proper size and orientation of the screws that are used for fusing the bones together. In addition, we try to localize the best bone region to fix these screws. To achieve these goals, the $2 \mathrm{D}-3 \mathrm{D}$ registration is introduced. The role of $2 \mathrm{D}-3 \mathrm{D}$ registration is to enhance the quality of the surgical procedure in terms of time and accuracy, and would greatly reduce the need for repeated surgeries; thus, saving the patients time, expense, and trauma. 


\section{TABLE OF CONTENTS}

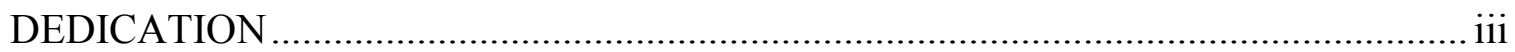

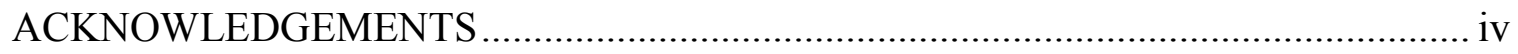

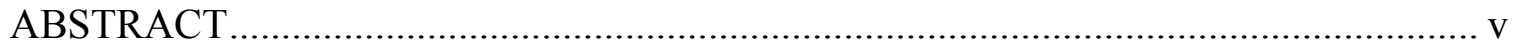

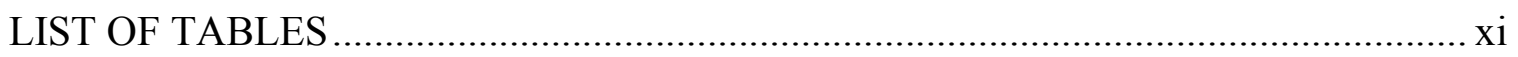

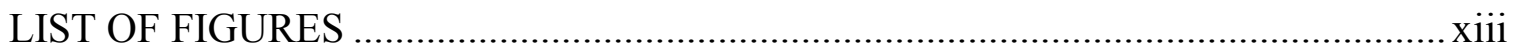

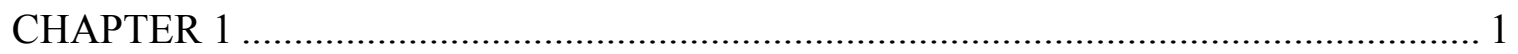

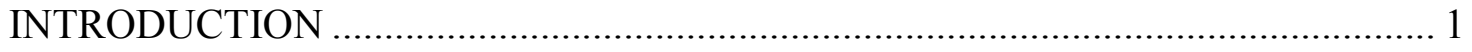

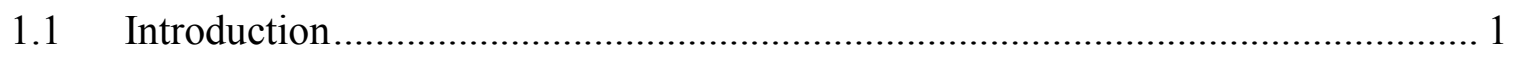

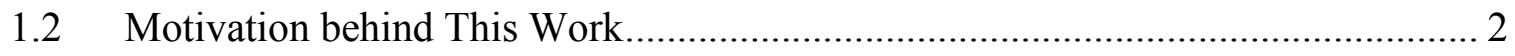

1.2.1 Shape Registration and Vertebral Body segmentation ................................... 2

1.2.2 Image Registration and Ankle Fusion..................................................... 4

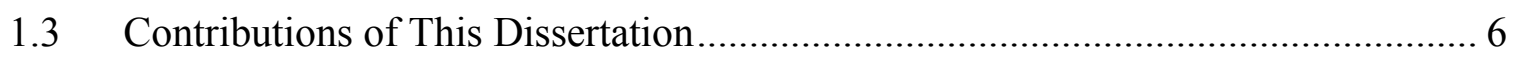

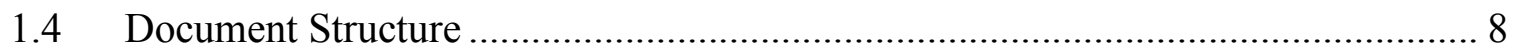

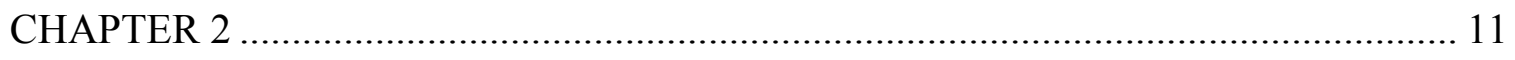

FUNDAMENTALS OF LEVEL SETS METHODS ............................................ 11

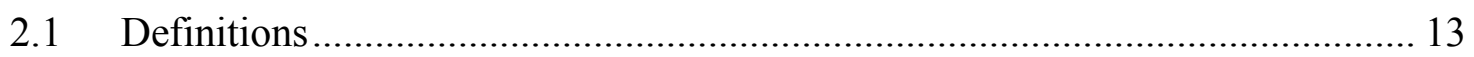

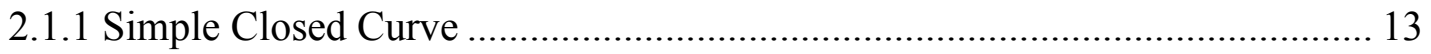

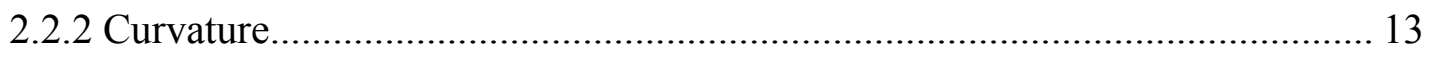

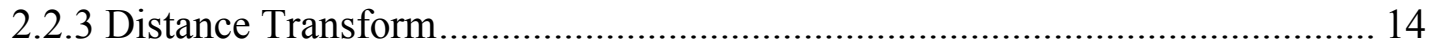




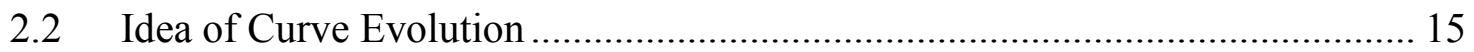

2.3 Representation of an Evolving Contour/Interface........................................... 16

2.3.1 Parameterized representation (Snakes) …………….................................. 16

2.3.2 Level sets representation (Geometric active contour) .................................... 18

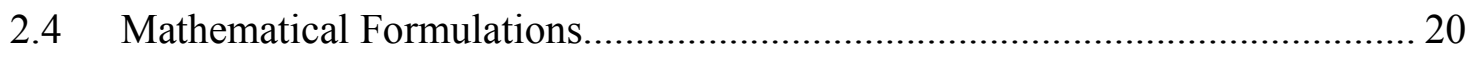

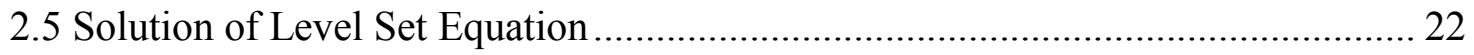

2.6 The Effects of Curvature and Viscosity Solutions............................................... 22

2.7 Motion under Curvature (Curvature Flow) ...................................................... 24

2.8 A level Set Formulation for Willmore Flow ....................................................... 25

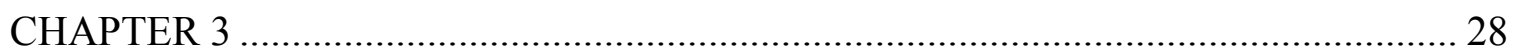

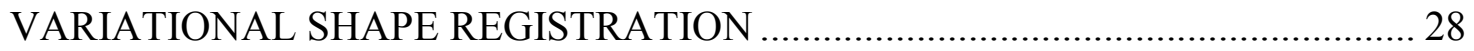

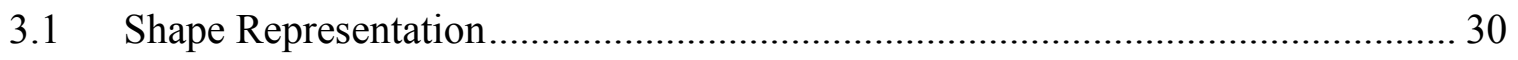

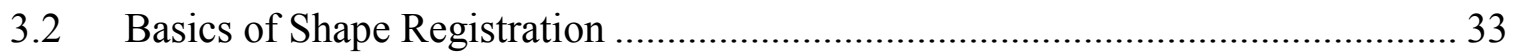

3.3 Global Registration of Shapes Using the Variational approach …………............ 35

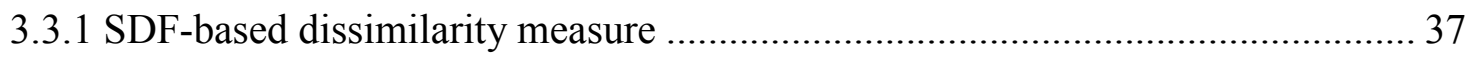

3.3.1.1 Two Dimensional Case: ................................................................................ 37

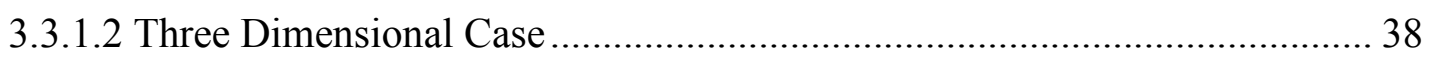

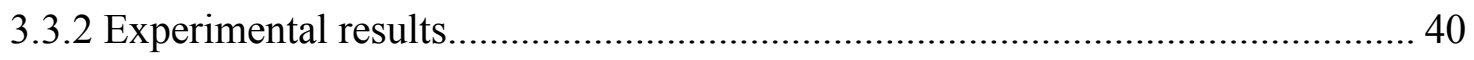

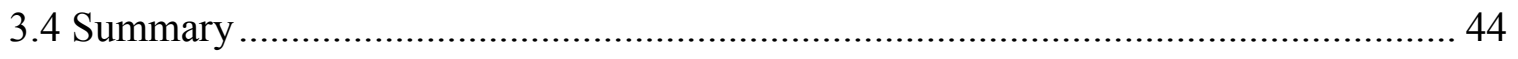

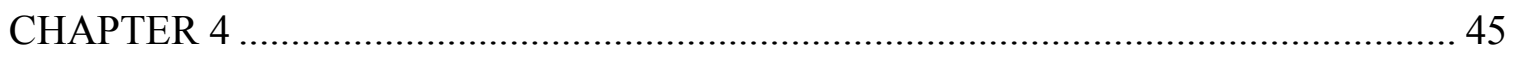

FUNDAMENTALS OF IMAGE REGISTRATION.................................................. 45

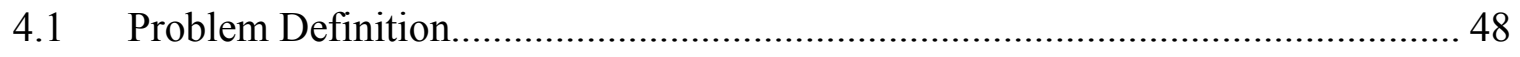

4.2 Intensity-Based Similarity Measures ……………............................................ 49 
4.2.1 Measures using only Image Intensities ............................................................ 50

4.2.1.1 Sum of Intensity Differences ................................................................... 50

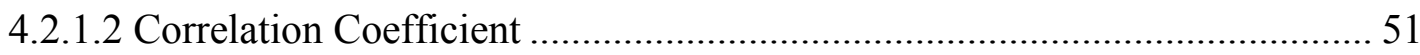

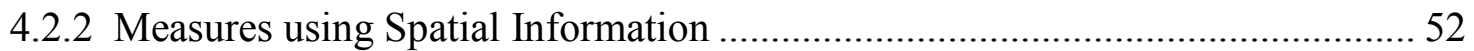

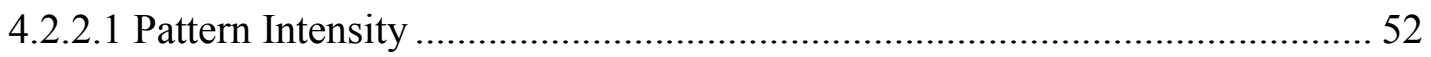

4.2.2.2 Gradient Correlation ....................................................................... 52

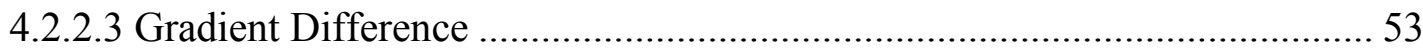

4.2.3 Histogram Based Measures.............................................................................. 53

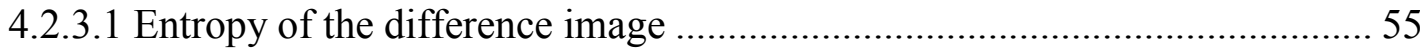

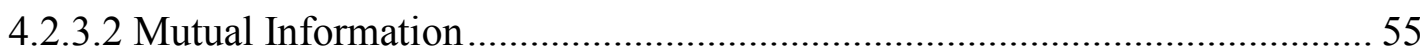

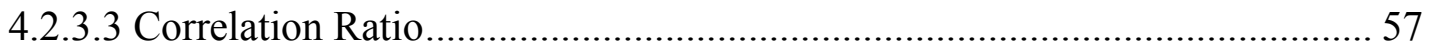

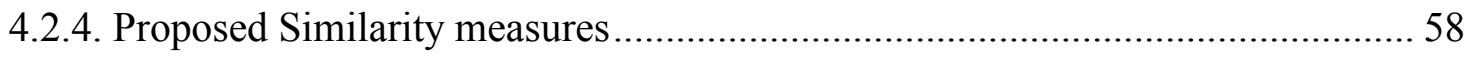

4.2.4.1 Exponential Correlation (EC) …….......................................................... 58

4.2.4.2 Individual Entropy Correlation Coefficient (IECC) …………………......... 59

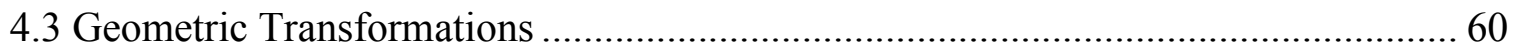

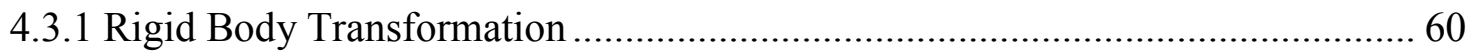

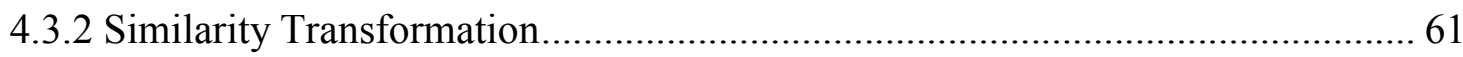

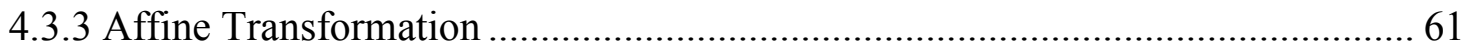

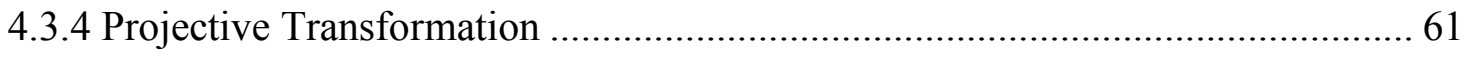

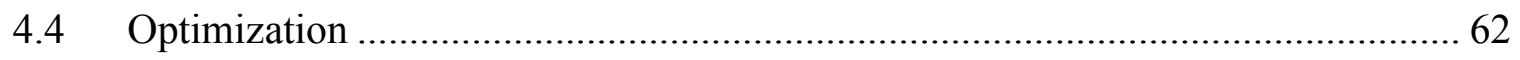

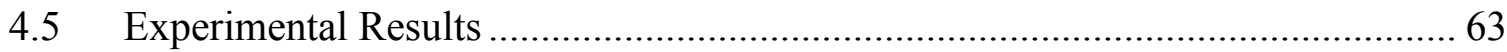

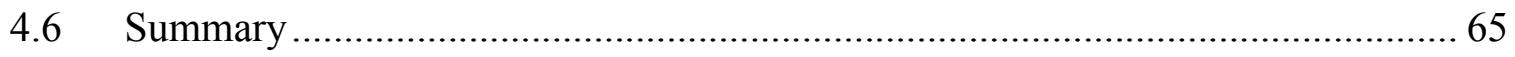

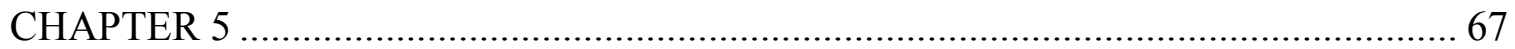


SHAPE BASED SEGMENTATION OF THE VERTEBRAL BODY....

5.1 Anatomy of the Spine Column and Osteoporosis................................................. 69

5.2 A review on VB Segmentation Methods and Related Work …............................ 72

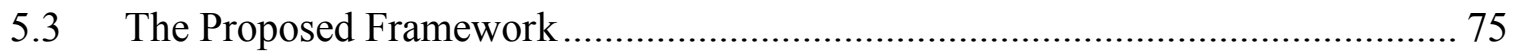

5.4 The Pre-processing Phase and Detection of ROI.................................................. 77

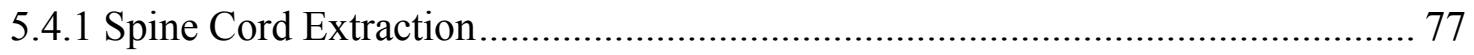

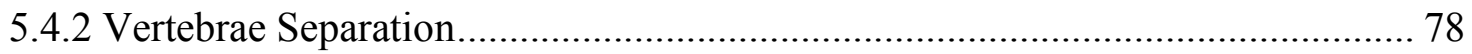

5.5 Two Dimensional Approach for VB Segmentation.............................................. 80

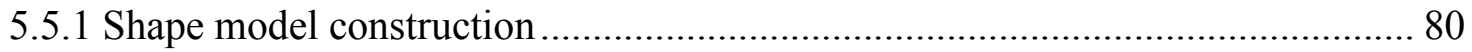

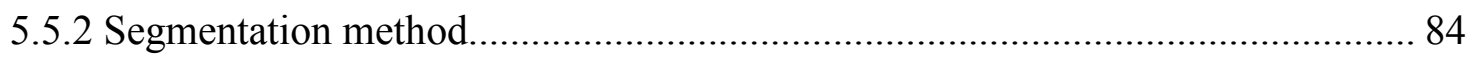

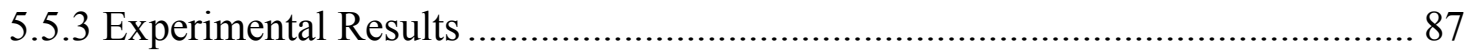

5.6 Three Dimensional Approach for VB Segmentation........................................... 93

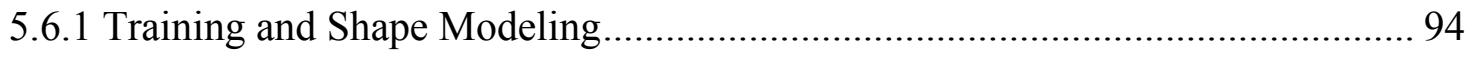

5.6.2 Simultaneous Segmentation and Registration .................................................. 97

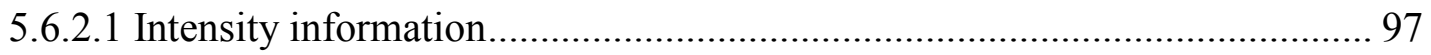

5.6.2.2 Embedding shape prior information ............................................................ 99

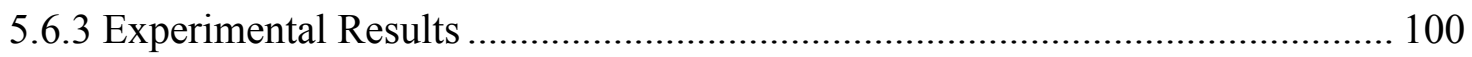

5.6.3.1 Results on Clinical CT Images ………………..................................... 102

5.6.3.2 Validation Using the Phantom .................................................................. 103

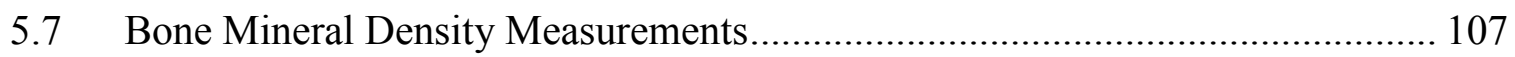

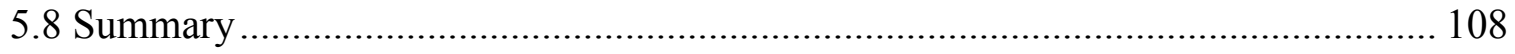

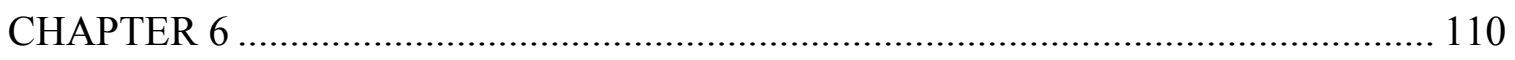

2D-3D REGISTRATION OF HUMAN ANKLE USING X-RAY AND CT ............ 110 


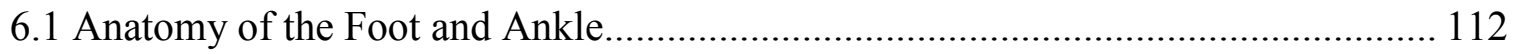

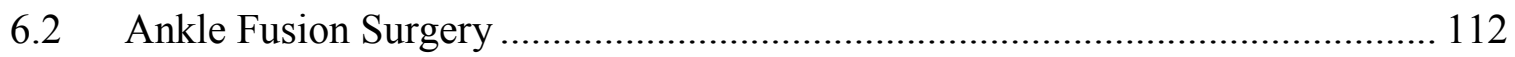

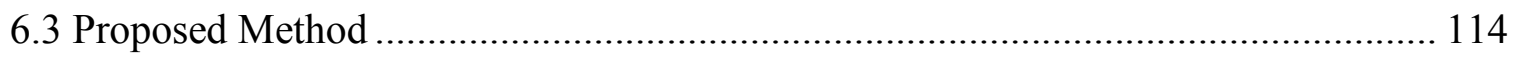

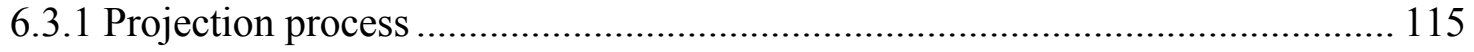

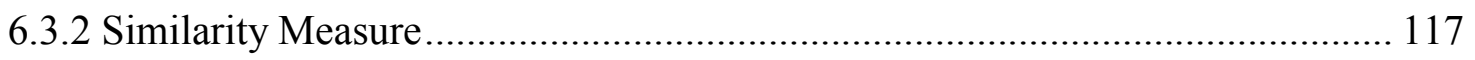

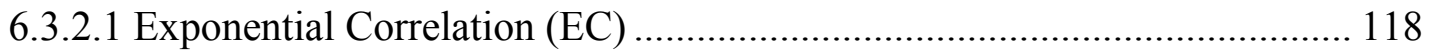

6.3.2.2 Individual Entropy Correlation Coefficient (IECC) ............................... 118

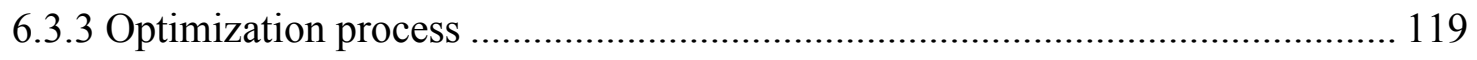

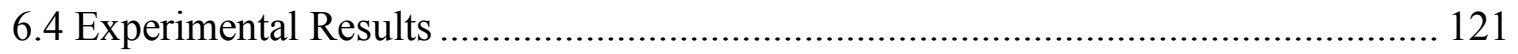

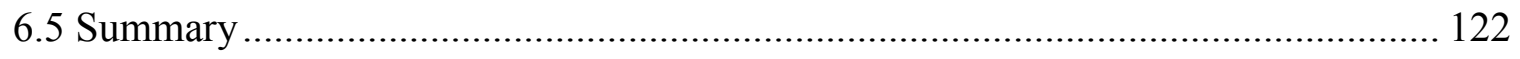

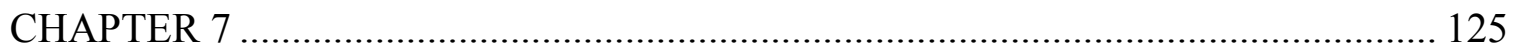

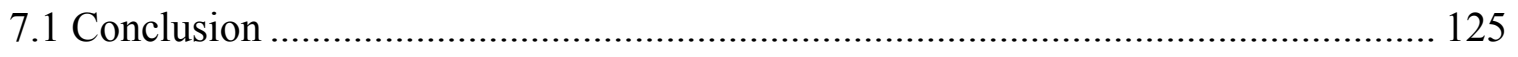

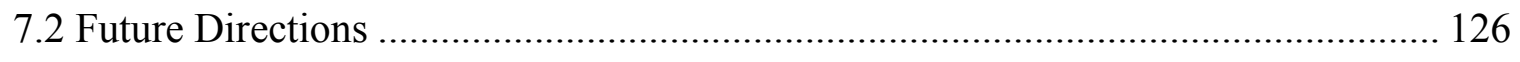

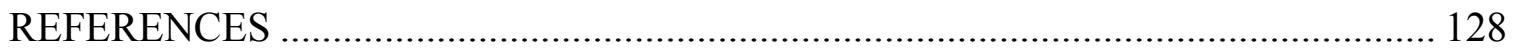

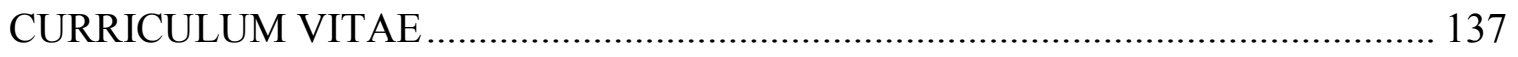




\section{LIST OF TABLES}

Table 3.1. Comparison of recovered parameters when using our model (M1) (2.6) vs. the use of similarity-based model (M2), i.e., $\mathbf{s}_{\mathbf{x}}=\mathbf{s}_{\mathbf{y}}=\mathbf{s}$, (Paragios et al., 2003[25]); GT stands

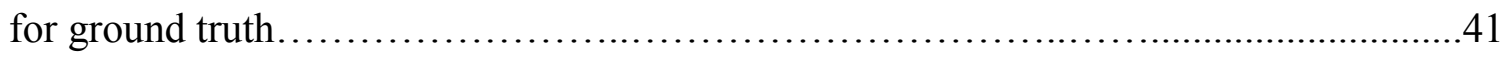

Table 4.1. Average and SD of the translation registration error for various types of image

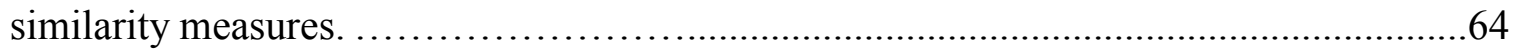

Table 4.2. Average and SD of the scaling registration error for various types of image

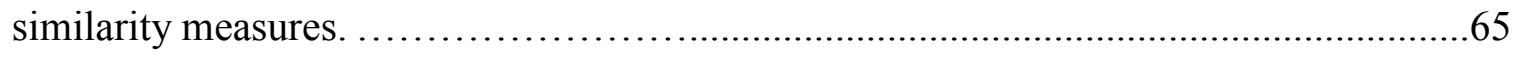

Table 4.3. Average excusion time (in miliseconds) for each similarity measure..............65

Table 5.1. Average DC (\%) and HD ( $\mathrm{mm})$ with standard deviation for segmentations of the clinical datasets using different methods. the average execution time of each method is summarized in the last row .104

Table 5.2. Average Relative Errors in BMD Measurement using different segmentation methods 104

Table 6.1. Correlation coefficient of the 2D-3D registration framework using NMI, EC and IECC for different views.

Table 6.2. Mean registration error and SD of the estimated parameters .124 


\section{LIST OF FIGURES}

Figure 1.1 Shape based segmentation of the human VB...........................

Figure 1.2 Components of the image-guided ankle/foot surgery $\ldots \ldots \ldots \ldots \ldots \ldots \ldots \ldots \ldots 4$

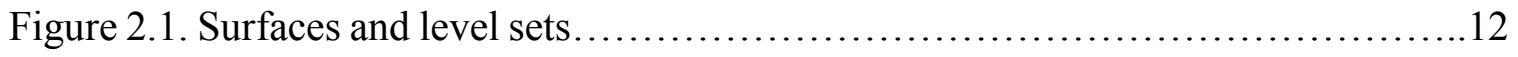

Figure 2. 2. (a) Simple closed cure, (b) Not simple curve............................13

Figure 2. 3. Illustrating distance transformation............................... 14

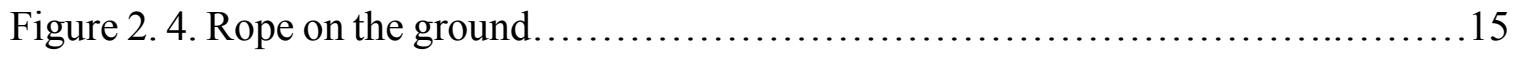

Figure 2.5. Parameterized representation of a contour............................. 16

Figure 2.6. Topological changes.......................................... 17

Figure 2. 7. The Level Set Surface (in red) plots the distance from each point (x, y) to the

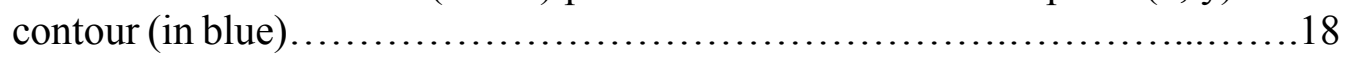

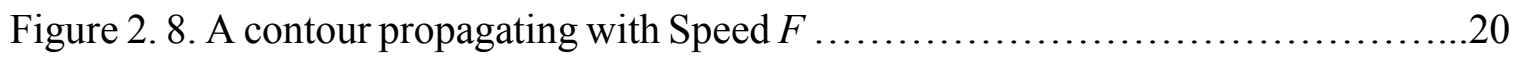

Figure 2.9. The two possible solution of sinusoidal evolution example.................23

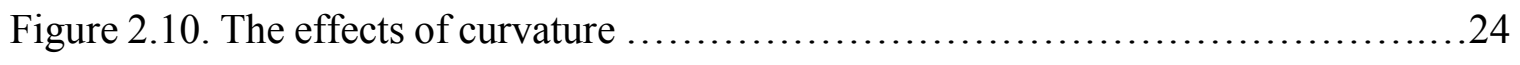

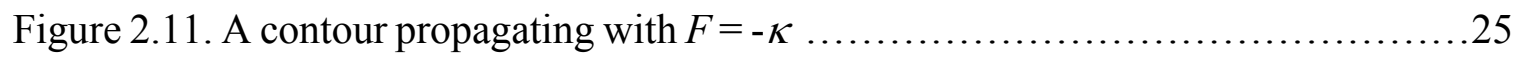

Figure 2.12. Another example for curvature flow ................................

Figure3. 1. Implicit shape representation using the signed distance function.............32

Figure 3.2. Registration example of a point from the source to the target shape...........34

Figure 3.3. Global registration. (a) Input Shapes (blue: source; red: target). (b) Registration results using the isotropic-scale based model, (c) Registration using the proposed model........................................................... 42 
Figure 3.4. Global registration. (a) Input Shapes. (b) Registration using homogeneous scale-based measure, (c) Registration using the proposed registration model (2.6). .42

Figure 3.5. Global Matching: Proposed Signed Distance- vs. VDF-based affine registrations [26]. (a) Input Shapes. (b) Affine Registration using the VDF representation (c) Affine Registration using new SSD criterion (2.6)...........43

Figure 3.6. Global registration of 3D shapes of two tooth shapes. (a) Input Shapes. (b) An intermediate state. (c) Registration using the proposed registration model (2.10). 43

Figure3.7 More examples for global registration of 3D shapes of various shapes. (a) Input Shapes. (b) An intermediate state. (c) Registration using the proposed

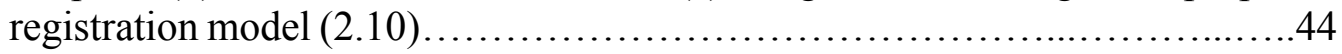

Figure 4.1. Block diagram of a typical image registration algorithm....................46

Figure 4.2. Classification of image similarity measures............................50

Figure 4.3. Joint and individual histograms for a synthetic and an X-Ray image. .54

Figure 5.1. The region of interest in the experiment, (a) The anatomical structure of the spine, (b) The red color shows the VB region. Bone Mineral Density (BMD) measurements and Fracture Analysis (FA) are restricted to the VB...........68

Figure 5.2. Typical challenges for vertebrae segmentation: (a) Inner boundaries. (b) Bone degenerative disease. (c) Osteophytes. (d) Double boundary, (e) weak bone edges, (f) osteophytes, and (g-h) low resolution

Figure 5.3. The sagittal view of the spine column. There are five regions of the spine column: cervical, thoracic, lumbar, and sacrum .70

Figure 5.4. Differences of the healthy and osteoporosis bone .71

Figure 5.5. Three different bone tissues. (a) Healthy, (b) osteopenia, and (c) osteoporosis bones. .71

Figure 5.6. A General block diagram of the proposed framework .75

Figure 5.7. (a) The template used for the Matched filter, (b-d) a few images of automatic VB detection. The green line shows the detection of VB region. .78 
Figure 5.8. Examples for the extraction of the spinal cord on a data set (a) The detected VB region, (b) The refined data to extract the spinal processes and ribs. and (c) The cropped data to reduce the size of the image.................................78

Figure 5.9. The separation of the VB region. (a) 3D view of three adjacent VB, (b) automated placement of 4 seeds on cortical bone and disc,(c) The histograms (red lines) and the thresholds (black lines) calculation along each detected seed, (d) separation of VB shown with red color. .79

Figure 5.10. An example of the initial labeling. (a) Original CT image, (b) detection of the VB region using MF, (c) the initial labeling, $f^{*}$ and (d) the SDF of the initial segmentation ( $\left.\mathrm{f}^{*}\right)$ which is used in the registration phase. Red color shows the zero level contour...................................................80

Figure 5.11. The proposed framework shape-based segmentation. .81

Figure 5.12. Comparison between the intensity based segmentation (initial labeling) using: (b) Scalar level sets model [64], and (c) graph cut method [61] .............90

Figure 5.13. Segmentation results of three different methods: (i) using graph cuts only, (ii) Method described in [58], and (iii) The 2D-PCA based segmentation.

Figure 5.14. Segmentation results of the ESP under different noise levels (i) using graph cut only. (ii) The algorithm (graph cut + shape prior). The red and yellow colors show the contour of the gold standards and segmented regions

Figure 5.15. Segmentation results with various shape initialization. (i) the initial shape prior, and (ii) is the final results. The red and yellow colors show the contour of the gold standards and segmented regions . .91

Figure 5.16. (a) The average segmentation accuracy of different segmentation methods on $500 \mathrm{CT}$ images under various signal-to-noise ratios.(b) The effect of choosing the number of the projected training shapes $N$ on the segmentation accuracy.93

Figure 5.17. Block diagram of the proposed simultaneous segmentation and registration method for human vertebral bodies (VB) extraction from CT images. The framework contains two main components; the training phase and the segmentation phase. A pre-processing step is used to eliminate the user interaction and reduce the execution time. .94

Figure 5.18. Constructing the shape prior volume. $\left\{\mathrm{VB}_{1}, \cdots, \mathrm{VB}_{M}\right\}$ training $\mathrm{CT}$ slices of different data sets. ( $M$ represents the number of training data sets). Last column shows the shape prior slices with variability region. .96 
Figure 5.19 (a) A 3D view of the 3D shape prior. (b) Different 3D views for the iso-surfaces $\mathbf{C}_{\boldsymbol{d}_{\boldsymbol{p}}}, \boldsymbol{p} \in \mathcal{V}$. Green color represents the object region $\boldsymbol{O}$, yellow color is the variability region $\mathcal{V}$, gray waves represent the the iso-surfaces $\mathbf{C}_{\boldsymbol{d}_{\boldsymbol{p}}}$, and red contour is the object/variability surface $\mathbf{C}_{\boldsymbol{O} v}$. (c) The probability of the object and background in the variability region................................ 96

Figure 5.20. (a) In the segmentation quality measurements, there are 4 regions to be considered as: True positive (TP), false positive (FP), true negative (TN), and false negative (FN). (b) The calculation of the HD between the red line $\mathrm{X}$ and the blue line Y. (c) Average geometrical dimensions of the lumbar vertebrae according to [31], all measurements in millimeters (mm). .102

Figure 5.21. 2D Axial projections of various stages of level set evolution for three different vertebrae using proposed method A1, (a) initial disk, (b) intermediate stage, (c) the final stage, and (d) the resultant 3D vertebral body...

Figure 5.22. 2D projections of the 3D Segmentation method in (a) Sagittal, (b) Coronal, and (c) Axial views for different examples from the clinical data sets. (The red and yellow colors show the contour of the ground truths and segmented regions, respectively) 105

Figure 5.23. An example for the 3D segmentation of clinical data sets overlaid with ground truth (yellow) using different methods: (a) Proposed, A1, (b) Graph cuts with shape prior, A2, (c) AAM, A3, (d) Chen-Vese with shape prior, A4, and (e) The results of level sets, A5. The red color represents the misclassified voxels. .106

Figure 5.24. 3D segmentation results of ESP using the four different methods: (a) the result proposed method A1, (b) the result of Graph cuts with shape prior A2, (c) the result of Chen-Vese with shape prior A4, and (d) the result of level set method A5. The red color represents the misclassified voxels. 106

Figure 5.25. The average segmentation accuracy (DC) of different segmentation methods on ESP dataset under various noise level. The results show that the proposed method is robust under various noise levels. ... .107

Figure 5.26. Relative errors of BMD measurement of each method. The BMD measurement of the method (A1) has the lowest error and standard deviation.108

Figure 6.1. Anatomy of the human foot. .111

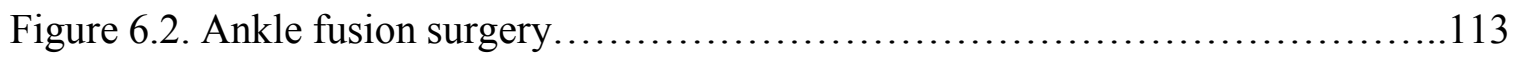

Figure 6.3. The block diagram of 2D-3D registration process. 115 
Figure 6.4. (a) Shear-warp factorization idea. (b) Examples for DRR based on SWF. (c) The initialization scenarios..........................................120

Figure 6.5. 2D-3D Registration results for different examples with different views for one of the clinical datasets using IECC as a similarity measure: (a) is the reference image (represents the intra-operative X-ray generated by ray-casting algorithm) (b) is the initial floating (synthetic) image, generated by SWF algorithm (c) is the final floating image after registration, (d) is checkerboard representation before registration and (e) after registration............................. 123 


\section{CHAPTER 1}

\section{INTRODUCTION}

"If we knew what it was we were doing, it would not be called research, would it?"

- Albert Einstein (1879 - 1955)

Shapes registration is an important problem in computer vision, computer graphics and medical imaging. It has been handled in different manners in many applications like shape-based segmentation, shape recognition, and tracking. On the other hand, image registration is the process of overlaying two or more images of the same scene taken at different times, from different viewpoints, and/or by different sensors. Many image processing applications like remote sensing for change detection, fusion of medical images, and computer-aided surgery need image registration.

\subsection{Introduction}

This work deals with two different applications in the field of medical image analysis. The first one is related to shape-based segmentation of the human vertebral bodies (VBs). The vertebra consists of the VB, spinous, pedicles, and other anatomical regions. Spinous processes, pedicles, and ribs should not be included in the bone mineral density (BMD) measurements since the BMD measurements are restricted to the VBs. The VB segmentation is not an easy task since the ribs and spinal processes have similar gray level information. Intensity based segmentation models may not be enough to obtain the optimum this target. Hence, a new shape based segmentation method is proposed. The second application is related to the field of computer-aided surgery, specifically on ankle 
fusion surgery. The long-term goal of this work is to apply this technique to ankle fusion surgery to determine the proper size and orientation of the screws which are used for fusing the bones together. In addition, try to localize the best bone region to fix these screws. To achieve these goals, the $2 \mathrm{D}-3 \mathrm{D}$ registration is introduced. The role of $2 \mathrm{D}-3 \mathrm{D}$ registration is to enhance the quality of the surgical procedure in terms of time and accuracy, and would greatly reduce the need for repeated surgeries; thus, saving the patient's time, expense, and trauma.

\subsection{Motivation behind This Work}

It is clear that, the work of this dissertation is directed into two different medical applications. The following sections give more details about these applications and why this work is needed.

\subsubsection{Shape Registration and Vertebral Body segmentation}

When images have noise, missing information, and occlusion problems, traditional segmentation methods will not be able to obtain desired segmentation. To solve the possible problems in the image, the shape prior information is integrated in the segmentation process. As shown in Figure 1.1, shape-base segmentation can be defined as the integration of the prior shape model into the segmentation via the shape registration process. In this matter, the prior shape model is obtained in advance using a number of training shapes of the object of interest. For the VB segmentation problem, there are limited publications dealing with this issue.

In this work, two different segmentation approaches are introduced. Both of them are obeying the variational shape-based segmentation frameworks. 


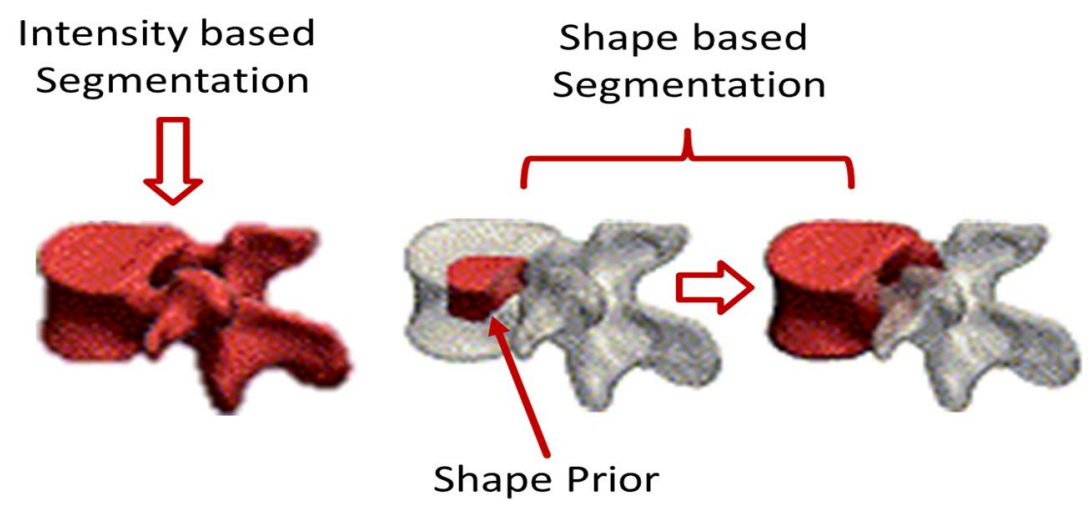

Figure 1.1 Shape based segmentation of the human VB.

The first approach deals with two dimensional (2D) case. This segmentation approach starts with obtaining the initial segmentation using the intensity/spatial interaction models. Then, shape model is registered to the image domain. Finally, the optimal segmentation is obtained using the optimization of an energy functional which integrating the shape model. The shape variations are modelled using two-dimensional principal component analysis (2D-PCA). The proposed method is tested on the synthetic and clinical images/shapes and it is shown to be robust under various noise levels and missing object information. The proposed shape based segmentation methods are less variant to the initialization.

The second one is a 3D simultaneous segmentation and registration approach. The information of the intensity are handled by embedding an edge-mounted Willmore flow into the level set segmentation framework. Then the shape variations are estimated using a new distance probabilistic model which approximates the marginal densities of the vertebral body and its background in the variability region using a Poisson distribution. The experimental results show that the segmentation accuracy of this framework are much 
higher than other alternatives. This study reveals that the proposed method is robust under various noise levels and completely eliminates the user interaction. Applications on bone mineral density (BMD) measurements of vertebral body are given to illustrate the accuracy of the proposed segmentation approach.

\subsubsection{Image Registration and Ankle Fusion}

An ankle fusion is a procedure that removes the damaged articular cartilage from the surfaces of the distal tibia, talus and fibula. The cut ends of the tibia and talus are brought together and held in place with screws and/or plates. The screws are typically stainless steel or titanium. Based on two plane C-arm fluoroscopy in the operating room, the doctor decides the size, the length, and the orientation of these screws.

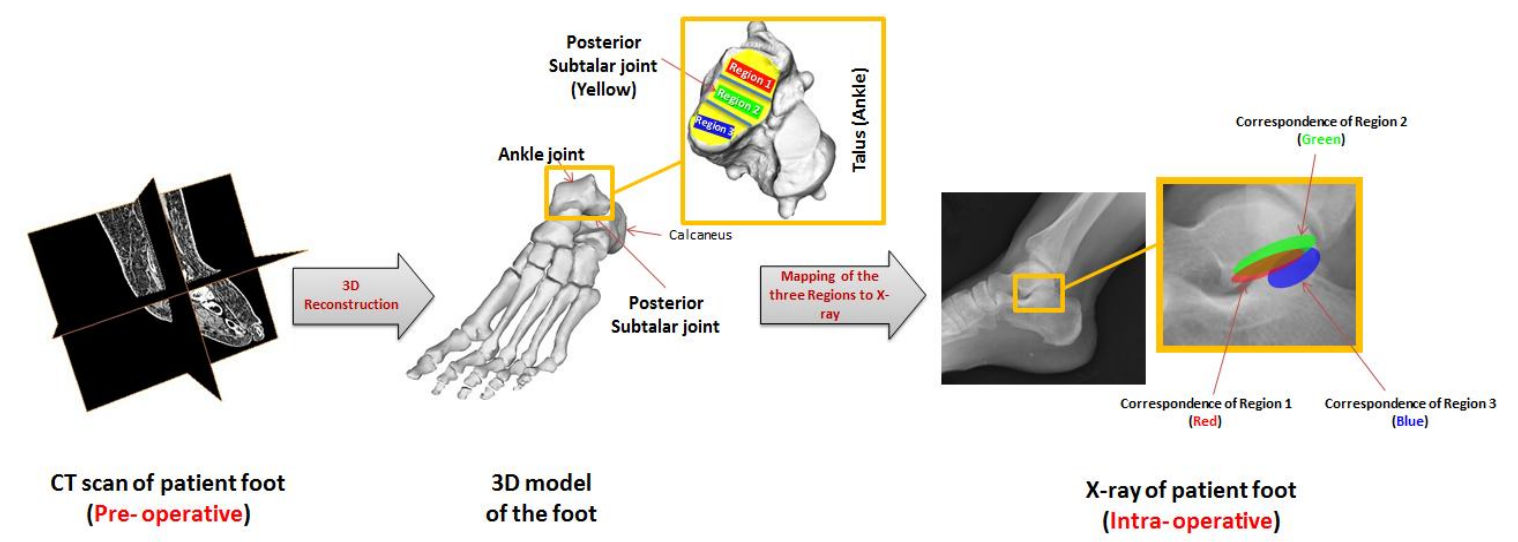

Figure 1.2 Components of the image-guided ankle/foot surgery

Yet there is one resounding issue with the current procedures to accomplish ankle fusion. The surgeon has limited visibility of the concave Subtalar joint below the talus, and too often the screws are too long or slightly at the wrong angle, resulting in screw penetration into the Subtalar joint, causing pain or joint damage. If this occurs, surgery is 
required to remove the screws, causing the patient additional time off work, surgery expense, and trauma.

We are proposing to create an image-guided tool that would allow the screws selected to be the proper length, and the angle selected to be the optimum angle, to fuse tibia to the talus, but not allow the screws to protrude through the talus into the Subtalar joint, as shown Figure1.1. The first step of that tool is the 2D-3D registration process. The process, in short, is aligning a 3D model based on pre-operative CT scans to corresponding 2D X-ray image acquired in the operation room (OR).

One of the key challenges of the $2 \mathrm{D}-3 \mathrm{D}$ registration problem is solving the correspondence problem; i.e., detecting features that are common between images in different modalities. A logical approach is to simulate one of the modalities given a complete reconstruction obtained from the other modality; i.e., simulate X-ray images from a CT volume. Given a real 2D image (i.e., X-ray image from the $\mathrm{C}$-arm) and candidates generated from another modality (i.e., CT), a transformation (T) may be estimated, based on a certain some similarity measure, which relates the real image to the best candidate; thus, correspondence between $2 \mathrm{D}$ to $3 \mathrm{D}$ is established. Therefore, a good similarity measure has to be identified that can quantify the quality of the alignment between the images and defining a procedure to modify and refine current estimates of the transformation parameters in a way that the similarity score is optimized. In other words, provided that we have a suitable similarity function, the best alignment parameters can be located with the help of an optimization procedure. Additionally, as the registration results 
are expected during the medical procedure, the computation time would also be constrained.

To achieve these goals, a new framework based on Exponential Correlation (EC) or Individual Entropy Correlation Coefficient (IECC) is proposed as new similarity measures for the 2D-3D registration process. It was tested on different clinical CT scans of human ankle and foot. Experiments demonstrated that EC-based framework is fast and performs almost as good as traditional similarity measures (that need more time) which is compatible with the time limitation of the interventional applications. From the accuracy point of view, the IECC-based framework is the most accurate system but at the expense of execution time.

\subsection{Contributions of This Dissertation}

This dissertation involves theoretical developments, system design and integration, as well as practical evaluation by professionals. Summarizing the main contributions of this work:

In Chapter 3 and 5:

- Two new shape based segmentation approaches are presented to isolate the human VBs.

- The first approach integrates intensity, spatial interaction, and shape prior information. It adopts the graph cut model for initial labeling and two-dimensional principal component analysis (2D-PCA) for shape construction.

- The second approach incorporates both shape information and an edge-mounted Willmore flow into the level set segmentation. 
- To get the optimal segmentation, a new vaiational energy function is formulated using the appearance models and shape constraints and iteratively minimized it using gradient descent.

- Applications on bone mineral density (BMD) measurements of vertebral body are given to illustrate the accuracy of the proposed segmentation approaches.

\section{In Chapter 4 \& 6:}

- New 2D-3D registration framework is specially adopted for ankle fusion.

- Specifically, adopting two novel similarity measures that quantify the quality of the alignment or (mapping) between 2D X-ray image and 3D CT volume.

- The framework was implemented based on shear-warp factorization (SWF) rendering techniques with Exponential Correlation (EC) and Individual Entropy Correlation Coefficient (IECC) as new similarity measures for the registration process.

- Experiments demonstrated that EC-based framework is fast and performs almost as much as NMI which is compatible with the time limitation of the interventional applications.

- Proposed approach can be considered as a step towards a robust computer-aided surgical station for ankle fusion surgery. 


\subsection{Document Structure}

This document is divided into two main parts. The first part (Chapters 2, 3 and 4) covers the theoretical background of the shape and image registration approaches. Chapter 2 briefly reviews on the fundamentals of level sets methods and mathematical foundations. Chapter 3 gives an introduction to the basics of shape registration, and discusses the fundamental definition of shape representation and the variational approaches. Chapter 4 deals with the image registration problem. It discusses the different classification of image registration algorithm and introduces two novel similarity measures: Exponential correlation (EC) and Individual Entropy Correlation Coefficient (IECC). The second part (Chapters 5 and 6) presents two proposed frameworks for two different applications in the medical imaging field. Chapter 5 introduces two novel shape based approach for the vertebral body (VB) segmentation framework. The first one deals with 2D case. In this approach, two-dimensional principle component analysis (2D-PCA) technique is exploited to extract the shape prior. The obtained shape prior is then registered into the image domain to develop a new shape-based segmentation approach. The experimental results show that the noise immunity and the segmentation accuracy of 2D-PCA based approach are much higher than conventional PCA approach. The other approach is a novel 3D vertebral body segmentation method in computed tomography (CT) images. The proposed approach depends on both intensity and shape information. The information of the intensity are handled by embedding an edge-mounted Willmore flow into the level set segmentation framework. Shape information is gathered from a set of training shapes. Then the shape variations are estimated using a new distance probabilistic model which approximates the 
marginal densities of the vertebral body and its background in the variability region using a Poisson distribution. Chapter 6 introduces a new framework for the 2D-3D registration of $\mathrm{CT}$ volumes and corresponding X-ray images. The objective is to apply this approach into ankle fusion. Different methods were used to evaluate registration quality of our system. Evaluation results confirm the degree of accuracy and robustness of the proposed framework. The proposed framework can be considered as a step towards a robust imageguided surgical station for ankle fusion Chapter 7 concludes the study and give insights for extensions and future work to be tackled during the dissertation. 
PART I

\section{THEORETICAL BACKGROUND}




\section{CHAPTER 2}

\section{FUNDAMENTALS OF LEVEL SETS METHODS}

Active contours are curves that deform within digital images to recover object shapes. They are classified as either parametric active contours or geometric active contours according to their representation and implementation. In particular, parametric active contours are represented explicitly as parameterized curves in a Lagrangian formulation. Geometric active contours are represented implicitly as level sets of two dimensional distance functions which evolve according to an Eulerian formulation. They are based on the theory of curve evolution implemented via level set techniques.

Parametric active contours are the older of the two formulations and have been used extensively in many applications over the last decades. A rich variety of modifications based on physical and non-physical concepts have been implemented to solve different shape estimation problems $[7,64,84]$. Geometric active contours were introduced more recently and were hailed as the solution to the problem of required topological changes during curve evolution $[85,86]$. Modifications and enhancements have been added to change their behavior or improve their performance in a variety of applications $[87,88$, 91-93], including a number of more global region based models which have appeared recently in the literature [94-99].

Geometric active contours or level set methods (Osher and Sethian, 1988) essentially find the shape without parameterizing it, so the curve description is implicit rather than explicit, by finding it as the zero level set of a function. The zero level set is the 


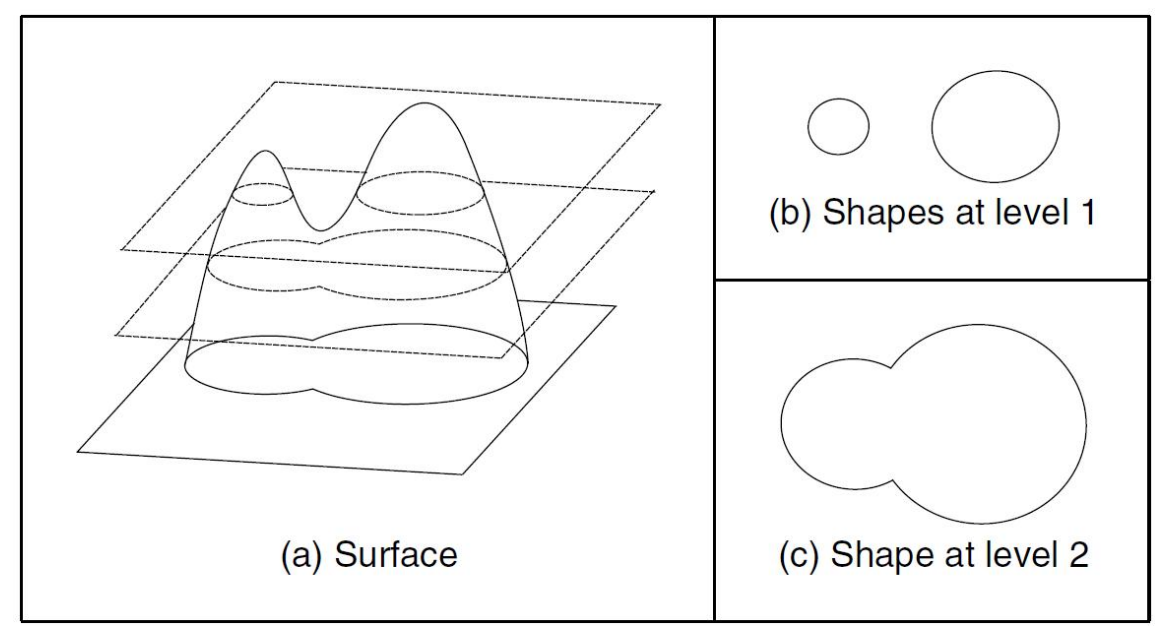

Figure 2.1. Surfaces and level sets.

interface between two regions in an image. This can be visualized as taking slices through a surface shown in Figure 2.1a. As we take slices at different levels (as the surface evolves) then the shape can split (Figure 2.1b). This would be difficult to parameterize, but it can be handled within a level set approach by considering the underlying surface.

At a lower level (Figure 2.1c), we have a single composite shape. As such, we have an extraction which evolves with time (to change the level). The initialization is a closed curve and we shall formulate how we want the curve to move in a way analogous to minimizing its energy. According to [86], the level set function is the signed distance to the contour. This distance is arranged to be negative inside the contour and positive outside it. The contour itself, the target shape, is where the distance is zero, at the interface between the two regions. Armed with these level set techniques, we can efficiently compute solutions to problems in geometry, fluid mechanics, computer vision, and materials sciences.

In this article, the level set methods will be deeply studied. We will concentrate on the basic theory and formulations of these techniques. Also we will touch the important 
applications of level sets in computer vision field. The following section covers some important definitions which are required as a bit of background to explain these techniques.

\subsection{Definitions}

As a starting point, it begins by recalling some important definitions. Then the representation of level sets will be analyzed in detail.

\subsubsection{Simple Closed Curve}

It is a connected curve that does not cross itself and ends at the same point where it begins - called also Jordan curve. Examples are circles, ellipses, and polygons. See

Figure 2.2

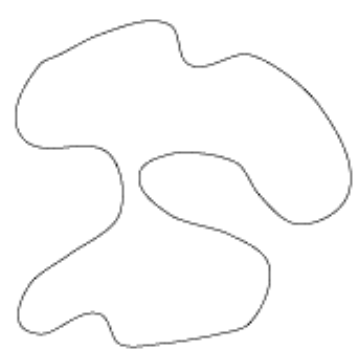

(a)

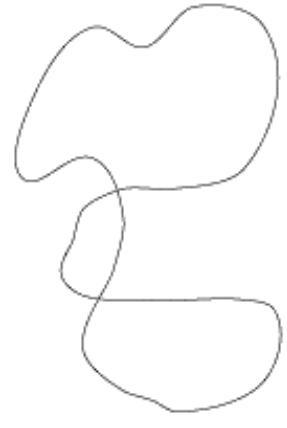

(b)

Figure 2. 2. (a) Simple closed cure, (b) Not simple curve.

\subsubsection{Curvature}

The curvature of a curve is, roughly speaking; the rate at which that curve is turning. Since the tangent line or the velocity vector shows the direction of the curve, this means that the curvature is the rate at which the tangent line or velocity vector is turning. For example, a circle has a constant curvature because it always is turning at the same rate; a smaller circle has a higher constant curvature because it turns faster. 


\subsubsection{Distance Transform}

It is possible to describe a shape not just by its perimeter, or its area, but also by it skeleton. Here we do not mean an anatomical skeleton, more a central axis to a shape. This is then the axis which is equidistant from the borders of a shape, and can be determined by a distance transform. In this way we have a representation that has the same topology, the same size and orientation, but contains just the essence of the shape. As such, we are again in morphology and there has been interest for some time in binary shape analysis.

Essentially, the distance transform shows the distance from each point in an image shape to its central axis. Intuitively, the distance transform can be achieved by successive erosion and each pixel is labeled with the number of erosions before it disappeared. Accordingly, the pixels at the border of a shape will have a distance transform of unity, those adjacent inside will have a value of two, and so on. This is illustrated in Figure 2.3, where Figure 2.3a shows the original shape with initial values of the distance and Figure $2.3 \mathrm{~b}$ shows the distance transform, where the pixel values are the distance. Here, the central axis has a value of 3 , as it takes that number of erosions to reach it from either side.

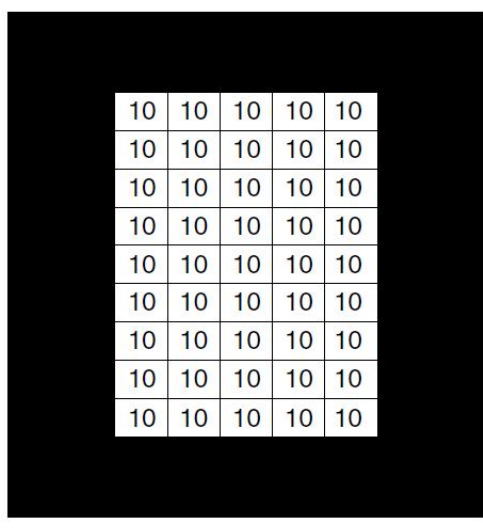

(a) Initial shape

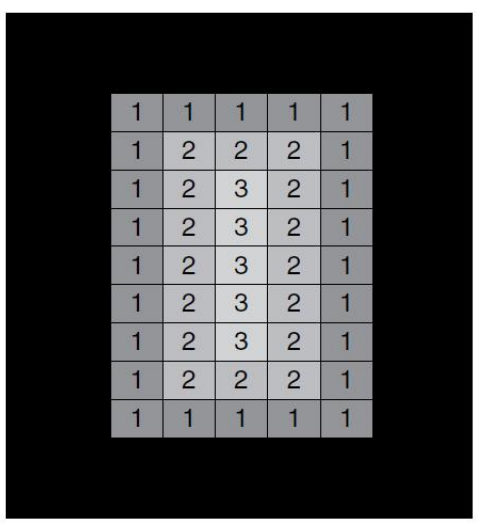

(b) Distance transform

Figure 2. 3. Illustrating distance transformation. 


\subsection{Idea of Curve Evolution}

Let a piece of rope - with two ends glued together- dropped to the ground. If the rope does not cross over itself, it will be considered as a simple closed curve. One defining characteristic of a curve is its curvature, which measures how fast the curve bends at any spot.

Now, suppose each piece of the curve moves perpendicular to the curve with speed proportional to the curvature. Since the curvature can be either positive or negative (depending on whether the curve is turning clockwise or counterclockwise), some parts of the curve move outwards while others move inwards. In Figure 2.4, the red arrows are where the curvature is negative, and the green arrows are where the curvature is positive: the arrows are of different lengths because the magnitude (or "strength") is larger at the green arrows than it is at the red ones.

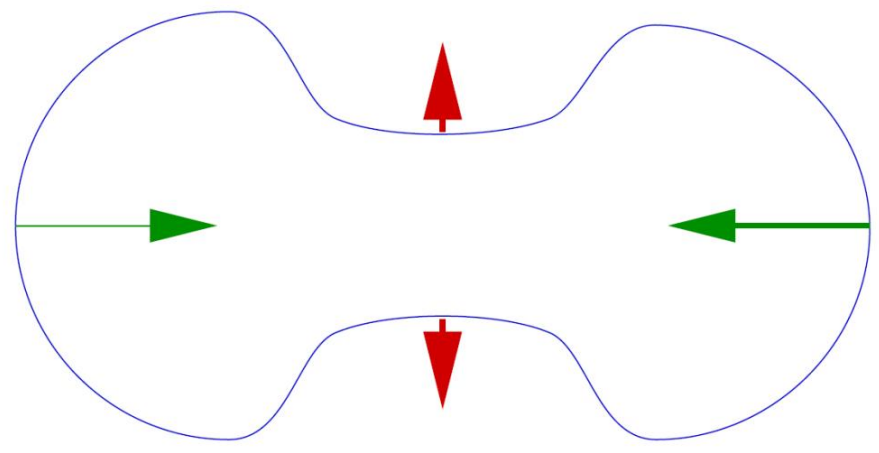

Figure 2. 4. Rope on the ground.

What happens to this curve as it moves according to this "motion by curvature"? If the initial curve is a circle, It is clear that each point on the curve races in towards the center, and the contour must collapse stay a circle and collapse to a single point. Regarding the 
rope, you can probably convince yourself that the shape relaxes itself and smooths out and becomes more circular. Actually, the motion by curvature is one component of many physical phenomena (for example, surface tension in a soap bubble and freezing rates at the edge of a snowflake both depend on the curvature at a point). Now, let us try to build a computer model of what happens to an evolving contour moving under its curvature.

\subsection{Representation of an Evolving Contour/Interface}

\subsubsection{Parameterized representation (Snakes)}

In order to move a contour, we need first a good way to describe it. One of these ways is called "curve parameterization". Suppose we try to use this parameterized representation of a contour as the backbone of a numerical algorithm. We can walk around the curve, and plant a blue dot at regular intervals. These dots, together with the ropes that connect them together, form a discrete view of the boundary; see Figure 2.5.

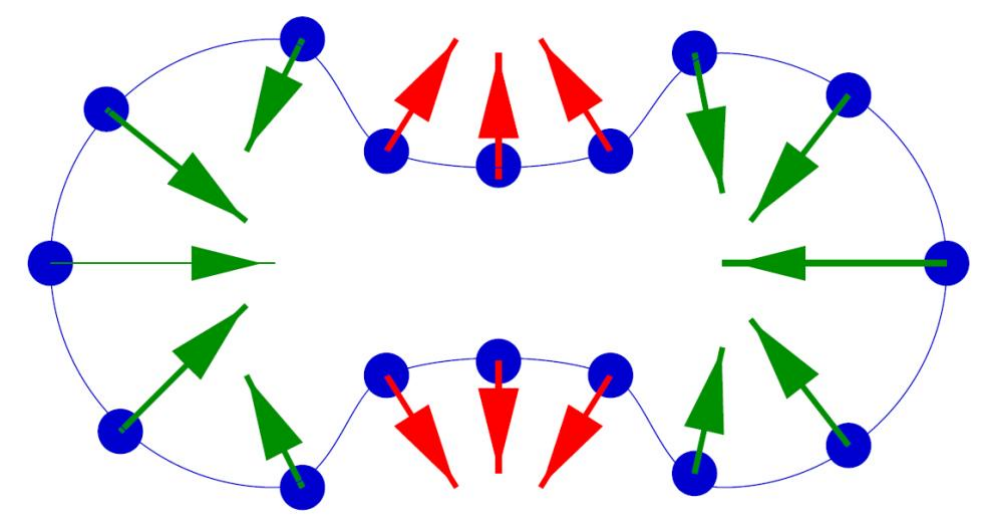

Figure 2.5. Parameterized representation of a contour.

Recall that the length and direction of the arrows is determined by the local curvature. The strategy is to advance the positions of the dots according to the arrows, recalculate new 


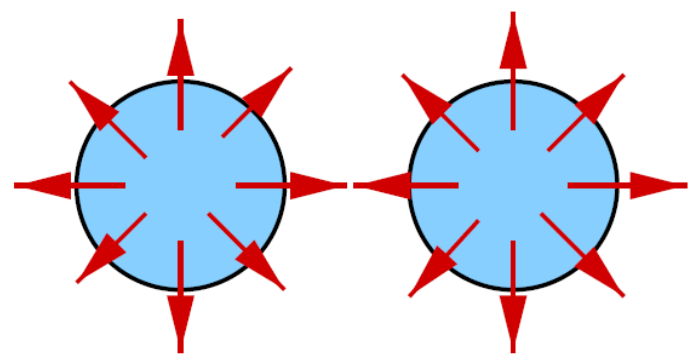

Initial Flame

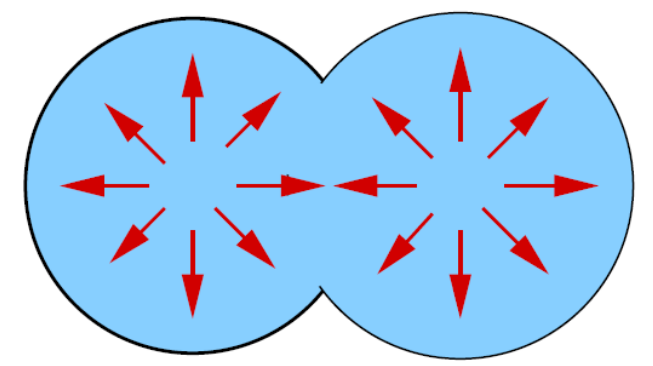

Later in Time

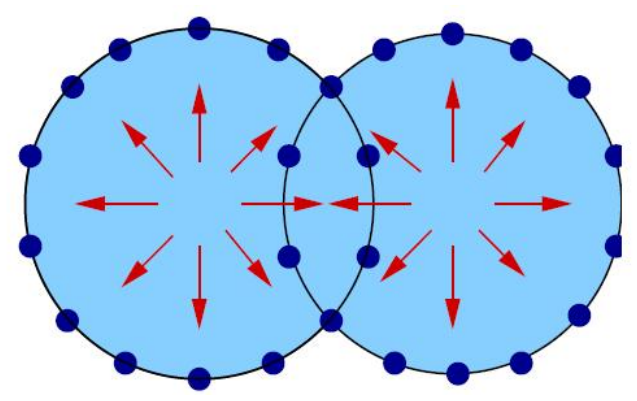

Figure 2.6. Topological changes.

arrows, and then advance the dots again. Unfortunately, there are several flaws in this approach, some inconvenient, some fatal. A look at the figure reveals an inconvenient one: the dots try and cross over themselves, and it becomes hard to keep the connecting ropes organized. A solution is to stop the advancement periodically, re-walk along the curve, and drop new equi-spaced dots. However, doing this for a propagating surface in three dimensions is very complex. A more serious problem comes when the evolving boundary attempts to change its topology. Taking a slight detour, consider two separate circular flames, each burning outwards at a constant speed, as shown in Figure 2.6. As the two separate flames burn together, the evolving contours merge into a single propagating front. However, a numerical algorithm based on a discrete parameterization runs into real trouble. The two pairs of dots located inside the burned region must somehow be removed if we 
want to track the true "edge" of the expanding flame. Trying to systematically determine which dots to remove is a confusing task. On the other hand, doing same algorithm in three dimensions is very complex.

\subsubsection{Level sets representation (Geometric active contour)}

Rather than follow the contour itself, the level set approach instead takes the original contour and adds an extra dimension to the problem. We are going to re-introduce a coordinate system, using the $x y$ plane which contains the contour, and a $z$ direction to measure height.

Suppose there is a function $z=\varphi(x, y, t=0)$, to take as input a point $(x, y)$, and assigns a height $z$. Where $z$ is the distance from $(\mathrm{x}, \mathrm{y})$ to the nearest point on the contour at time $\mathrm{t}$ $=0$. This builds a surface (shown in red at Figure 2.7) with the property that it intersects the $x y$ plane exactly at the contour. The red surface is called the level set function, because it accepts as input any point in the plane and hands back a height as output. The blue contour is called the zero level set, because it is the collection of all points that are at height zero.

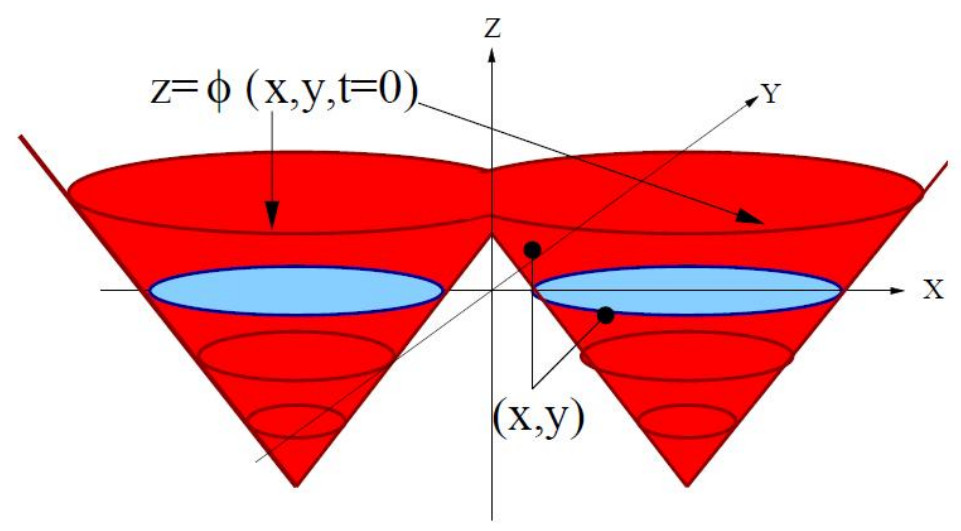

Figure 2. 7. The Level Set Surface (in red) plots the distance from each point (x, y) to the contour (in blue). 
The plan is to figure out how to change the height of the surface $\varphi(x, y, t)$, in time to match the evolution of the contour. The goal is to let the level set function expand, rise, fall, and do all the work. Then, to find out where the contour is at any time, we can simply cut the surface at zero height, or, plot the zero contour. It is clear that, level set methods exchange a geometric, moving coordinate representation for a fixed coordinate perspective where each point $(x, y)$ adjusts its value to measure the distance to the evolving interface/contour.

At first glance, it might seem impractical to take the problem of a moving curve and trade it in for a moving surface. More dimensions usually mean more work. The reason the extra dimension is so powerful is that, rather than track dots around which can collide and stretch apart, we can now stand at each point $(x, y)$ and adjust the height of the level set function. This means, for example, that the topological problems have vanished; two expanding flames which merge into one simply means that the zero level set at a particular time becomes one curve rather than two.

One of the most advantages of the level set approach is that nothing is changed for interface problems in three (or more) dimensions: while slightly harder to visualize, the strategy is still the same. First, embed the evolving surface in one higher dimension. In the case of a propagating surface, this would mean using a time-dependent function $\varphi(x, y, z, t$ $=0$ ) in four-dimensional space. Then, adjust this higher dimensional function corresponding to motion of the interface, and compute the "zero" level set to find the position of the propagating interface.

The following sections discuss more technical details about level set methods and its mathematical formulations 


\subsection{Mathematical Formulations}

Suppose you are given an interface separating one region from another (either a closed curve in two dimensions or a closed surface in three dimensions), and a speed $F$ that tells you how to move each point of the interface. Here, $F$ can depend on all sorts of complex physics, such as heating on either side of the interface, or fluid mechanical effects. Regardless, we shall assume that the speed $F$ is handed to us, and gives the speed in the direction perpendicular to the interface. It is clear that any tangential component will have no effect on the position of the front.

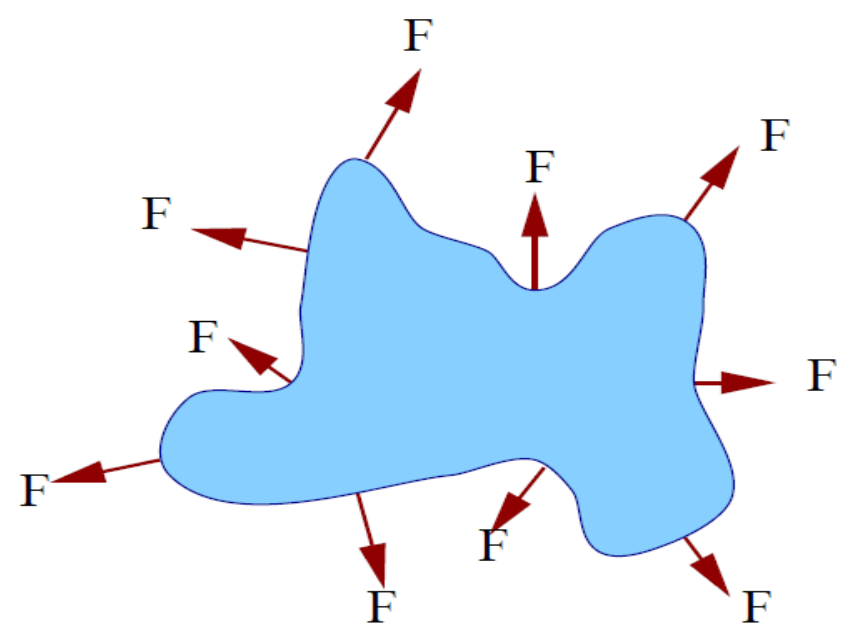

Figure 2. 8. A contour propagating with Speed $F$.

An initial value for the level set function $\varphi(\mathrm{x}, \mathrm{y}, \mathrm{t}=0)$ will be built based on the signed distance $d$ from each point $(x, y)$ to the initial contour, choosing a positive distance if we are outside the blue region, and a negative if we are inside. This constructs an initial value for the level set function $\varphi$. All that remains is to figure out how to adjust its value in time to match the evolving interface. 
Let us assume that the interface $C$ is controlled to change in a constant manner and evolves with time $t$ by propagating along its normal direction with speed $F$, where $F$ is a function of curvature and speed, according to:

$$
\frac{\partial C}{\partial t}=F \cdot \frac{\nabla \varphi}{|\nabla \varphi|}
$$

Here, the term $\frac{\nabla \varphi}{|\nabla \varphi|}$ is a vector pointing in the direction normal to the surface. The curve is then evolving in a normal direction, controlled by the curvature. At all times, the interface $C$ is the zero level set, so:

$$
\varphi(C(t), t)=0
$$

The level set function $\varphi$ is positive outside the region and negative when it is inside, and it is zero on the boundary of the shape. As such, by differentiation:

and by the chain rule we obtain

$$
\frac{\partial \varphi(C(t), t)}{\partial t}=0
$$

$$
\frac{\partial \varphi}{\partial C} \frac{\partial C}{\partial t}+\frac{\partial \varphi}{\partial t}=\mathbf{0}
$$

By rearrangement, and substitution from (2.1):

$$
\frac{\partial \varphi}{\partial t}=-F \frac{\partial \varphi}{\partial C} \cdot \frac{\nabla \varphi}{|\nabla \varphi|}=-F|\nabla \varphi|
$$

A little bit of algebraic manipulation produces the level set equation, namely:

$$
\varphi_{t}+F \sqrt{\varphi_{x}^{2}+\varphi_{y}^{2}}=0
$$

where the subscripts means that we are taking partial derivatives. 


\subsection{Solution of Level Set Equation}

The above level set equation is called an initial value partial differential equation, where it describes the time-evolution of a solution on the basis of an initial state, and partial deferential because the equation contains partial derivatives. This is a form of the Hamilton- Jacobi equation, which is a partial differential equation that needs to be solved so as to obtain the solution. One way to achieve this is by finite differences and a spatial grid (the image itself). We then obtain a solution that differences the contour at iterations $<n+1>$ and $<n>$ separated by an interval $\Delta t$ as:

$$
\varphi(i, j, \Delta t)^{<n+1>}=\varphi(i, j, \Delta t)^{<n>}-\Delta t\left(F\left|\nabla_{i j} \varphi(i, j){ }^{<n>}\right|\right)
$$

where $\nabla_{i j} \varphi$ represents a spatial derivative.

In the following sections, we will discuss a collection of schemes for solving general Hamilton- Jacobi and level set equations in a triangulated and finite element framework.

\subsection{The Effects of Curvature and Viscosity Solutions}

An illustration of the power of partial deferential equations comes from, consider the following example. Starting from the simple case of a constant speed function $F=1$, and a sinusoidal initial interface, consider two solutions to the problem. One called: The Swallowtail solution and the second called: The leading wave Solution. As illustrated in Figure 2.9, the two solutions are the same until a corner develops in the propagating interface, at which point one of them overlaps itself, while the other chooses only the leading wave. Intuitively, the "leading wave" solution seems like the physically correct one, especially in light of the earlier discussion about removing markers which do not lie 


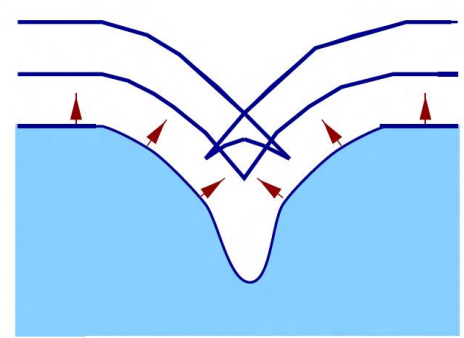

The Swallowtail Solution

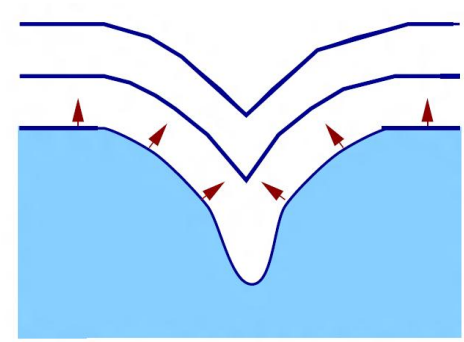

The Leading Wave Solution

Figure 2.9. The two possible solution of sinusoidal evolution example.

on the boundary between inside and outside. The problem now is: as soon as, the evolving front develops the sharp corner, all possibilities are off. We cannot evaluate the partial derivative at a place when the slope makes a sudden jump in direction. The solution comes from the mathematical theory of viscosity equation.

Loosely speaking, viscosity measures the ability of a fluid right damp sharp transitions and mute sudden changes. If you drop a marble in a jar of honey, the viscosity of the honey slows it down. This idea of viscosity will be used to smooth out the corner in the propagating interface. We can think of this as adding a little viscosity. With this in mind, let us consider a speed of the form $F=1-0.1 \kappa$, where $\kappa$ is the local curvature of the contour. $\kappa$ is defined to be:

$$
\kappa=\nabla \cdot N
$$

where $\boldsymbol{\nabla}$. Is the divergence operator, and $N$ is the normal direction. According to $[102],=\frac{\nabla \varphi}{|\nabla \varphi|}$. 


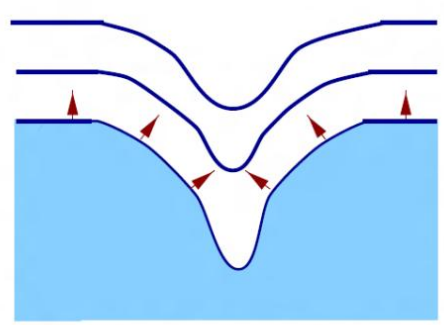

Some Viscosity: $F=1-.1 \kappa$

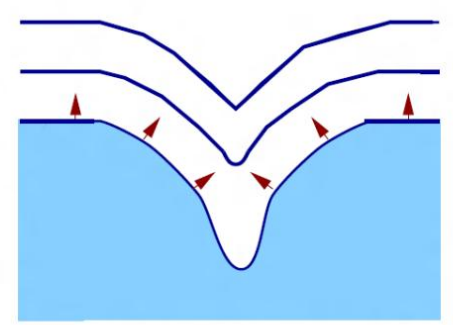

Less Viscosity: $F=1-.01 \kappa$

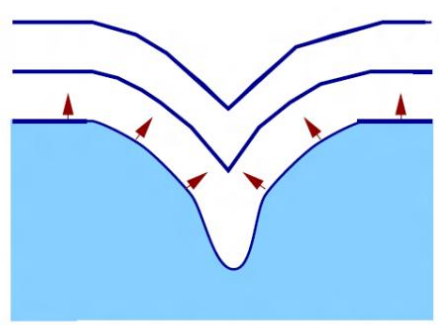

No Viscosity: $F=1$

Figure 2.10. The effects of curvature.

Now, Substitute this speed into the level set equation to produce:

$$
\varphi_{t}+\sqrt{\varphi_{x}^{2}+\varphi_{y}^{2}}=0.1 \kappa
$$

As observed from Figure 2.10, even though there is very little smoothing going on with such a small amount of curvature, it is enough to guarantee that a corner never develops. So, we can say that: the theory of viscosity solutions leads to a remarkable fact: if we take a sequence of problems, each with ever smaller viscosity, they will head towards the corner "leading wave" solution. This means that all we need to do is solve for the viscosity solution of the level set equation, and we are guaranteed to pick out the right topological evolving front. For more details about the viscosity solutions and level sets, please see [64].

\subsection{Motion under Curvature (Curvature Flow)}

As a first application of level set methods, we can revisit motion by curvature $\kappa$, and examine what happens to a closed curve moving with speed $F=-\kappa$ (the minus sign is chosen so that convex parts move in, and concave parts move out). We have seen that a circle must collapse smoothly to a point before it disappears, and argued that more complicated simple closed curves must smooth out. In fact, Grayson, [100], proved that 
every simple closed curve collapses smoothly to a single point, without crossing over itself. This is a remarkable theorem: no matter how complicated or convoluted a curve might be, it quickly relaxes itself into a circular object and shrinks down to a point. As shown in Figure 2.11 and 2.12, we show such curves shrinking to a circular object; from there, it is easy to believe that it shrinks to a point and disappears.
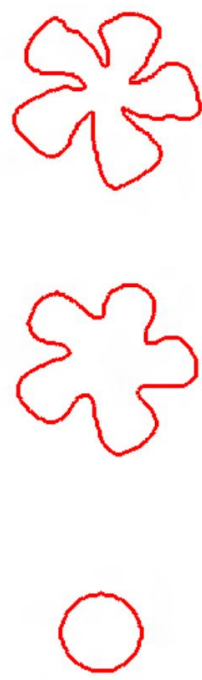
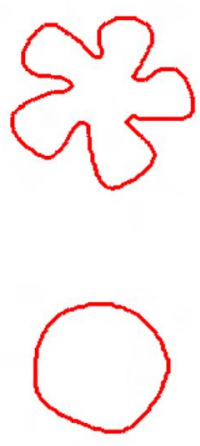

0
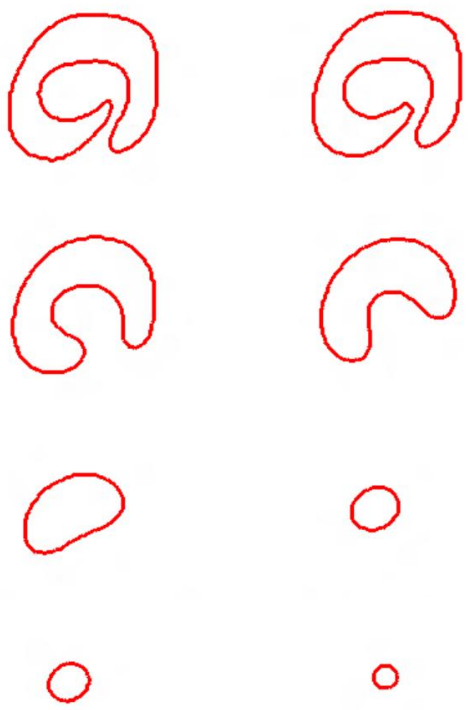

Figure 2.11. A contour propagating with $F=-\kappa \quad$ Figure 2.12. Another example for curvature.

\subsection{A level Set Formulation for Willmore Flow}

A level set formulation of Willmore flow is derived using the gradient flow perspective. Starting from single embedded surfaces and the corresponding gradient flow, the metric is generalized to sets of level set surfaces using the identification of normal velocities and variations of the level set function in time via the level set equation. The approach in particular allows to identify the natural dependent quantities of the derived variational formulation. Furthermore, spatial and temporal discretization are discussed and some numerical simulations are presented. 
The Willmore energy of a surface as a function of mean and Gaussian curvature, captures the deviation of a surface from sphericity. As such this energy and its associated gradient flow play an important role in digital geometry processing, geometric modeling, and physical simulation.

Let $M$ be a $d$-dimensional surface embedded in $\mathfrak{R}^{d+1}$ and denote by $x$ the identity map on $M$. Consider the energy

$$
E_{M}=\frac{1}{2} \int_{M} h^{2} d A
$$

where $h$ is the mean curvature on $M, \mathrm{i}$ e., $h$ is the sum of the principle curvatures on $M$. The corresponding $l^{2}$-gradient flow - the Willmore flow is given by the geometric evolution problem

$$
\frac{\partial x}{\partial t}=\Delta_{M} h(t) n(t)+h(t)\left\{\|S(t)\|^{2}-\frac{1}{2} h(t)^{2}\right\} n(t),
$$

which defines for a given initial surface $M_{0}$ a family of surfaces $M(t)$ for $t, 0$ with $M(0)=$ $M_{0}$. Here $S(t)$ denotes the shape operator on $M(t), n(t)$ the normal field on $M(t)$, and $\|$.$\| the$ Frobenius norm on the space of endomorphisms on the tangent bundle $M(t)$.

Now we consider $M(t)$ to be given implicitly as a specific level set of a corresponding function $\boldsymbol{\phi}(t): \Omega \rightarrow \mathfrak{R}$ for a domain $\mathfrak{R}^{d+1}$. Thus, the evolution of $\mathcal{T} M(t)$ can be described by an evolution of $\boldsymbol{\phi}(t)$. In this case, the level set equation (2.5) turns into the equation:

$$
\frac{\partial \phi}{\partial t}=-|\nabla \phi(t)|\left(\Delta_{M} h(t)+h\left\{\|S(t)\|^{2}-\frac{1}{2} h(t)^{2}\right\}\right)
$$

where $\Delta_{\Gamma} \boldsymbol{h}=\Delta \boldsymbol{h}-\boldsymbol{h} \frac{\partial \boldsymbol{h}}{\partial \boldsymbol{n}}-\frac{\partial^{2} \boldsymbol{h}}{\partial \boldsymbol{n}^{2}}$ is the Laplacian Beltrami operator on $h$ with $n=\frac{\boldsymbol{\nabla} \boldsymbol{\phi}}{|\boldsymbol{\nabla} \phi|}, S=(\mathrm{I}$ 
$-n \otimes n)(\nabla \times \nabla) \boldsymbol{\phi}$ is the shape operator on $\boldsymbol{\phi}$, and $\|\boldsymbol{S}\|$ is the Frobenius norm of $S$.

Let us emphasize that different from second order geometric evolution problems, such as mean curvature motion, for fourth order problems no maximum principle is known. Indeed, two surfaces both undergoing an evolution by Willmore flow may intersect in finite time. Hence, a level set formulation in general will lead to singularities and we expect a blow up of the gradient of Á in finite time. If one is solely interested in the evolution of a single level set, one presumably can overcome this problem by a re-initialization with a signed distance function with respect to this evolving level set.

In this dissertation, a novel 3D vertebral body segmentation method in computed tomography (CT) images will be presented. This approach depends on both intensity and shape information. The information of the intensity are handled by embedding an edgemounted Willmore flow into the level set segmentation framework. Shape information is gathered from a set of training shapes. Then the shape variations are estimated using a new distance probabilistic model which approximates the marginal densities of the vertebral body and its background in the variability region using a Poisson distribution. More details about this algorithm will be discussed in Chapter 5 . 


\section{CHAPTER 3}

\section{VARIATIONAL SHAPE REGISTRATION}

Shape registration is a fundamental problem in computer vision, and a core component in various medical applications. Recognition, tracking and retrieval are some other examples of applications that may benefit from shape alignment. Numerous approaches have been developed to solve this problem, each of which has its strengths and its limitations. These approaches can be categorized based on three main aspects: 1) the selected model to represent the shape, 2) the transformation model, and 3) the mathematical framework chosen to recover the matching parameters.

Shape representation is handled differently in each application. For instance, in [1], the authors choose to represent the shape to be registered as the zero level sets of distance functions in a higher dimensional space. This implicit representation is known to be invariant to translations and rotations, and performs accurately in the case of homogeneous scales. To account for anisotropic scales, the authors proposed to maximize an information based criterion, namely the Mutual Information (MI), in the embedding space. Hong [2] proposed a new shape representation algorithm and showed its potential in image matching and segmentation. This algorithm is based on integral kernels and represents a shape as the area of intersection between the kernel and the inside and outside of the shape. The kernel width has a major effect on the method output and its appropriate selection was not clearly discussed. In [3], an approach was proposed to solve the shape registration problem by using a volumetric representation of shapes through vector level sets. The authors claimed that this representation is more suitable for scale variations. In [4], the authors used the 
point-set representation for shapes in order to solve the non-rigid registration problem. This representation, also known as a cloud of points representation, was also used in [5], where the authors proposed to describe each sample point by a "shape context", and use this descriptor to match shapes for object recognition purposes. Other methods have been considered to represent shapes in different applications. These methods include, among others, medial axis [6] and Fourier descriptors [7].

Transformation models can be divided into two classes: global and local [8]. The global transformation models are usually defined by a small set of parameters, e.g., rigid (rotations and translations), similarity (rigid plus homogeneous scale), and affine, which in addition to rotations and translations, account for anisotropic scales and/or shearing. Such transformations can be used alone to efficiently align two shapes. However, in the case of local deformations, more complex and general transformations are to be considered in order to establish dense correspondences between the two input shapes.

Different techniques were developed to solve the non-rigid shape registration problem. In [9], the authors presented the Thin Plate Spline-Robust Point Matching (TPSRPM) algorithm, to jointly estimate the rigid and non-rigid transformations between two clouds of points that may be of different sizes. Thin plate splines were also adopted in [5] where corresponding points are determined by iteratively minimizing the overall shape context distances, and the TPS transformation is re-estimated at each iteration to recover the local transformation maps. More recently, Chen and Bhanu [10] introduced a global-to local procedure to align non-rigid shapes. The shape context descriptors are used to recover a global similarity transformation, while the local deformation is performed within an 
optimization formulation, in which the bending energy of TPS transformation is incorporated as a regularization term. In [11], Yezzi and Soatto proposed a definition of motion for a deforming object and introduced the notion of "shape average", which allowed the derivation of new algorithms to align non identical shapes using region-based techniques. Zheng and Doermann [12] proposed a relaxation labeling-based point matching algorithm for non-rigid shapes. The authors formulated point matching as a graph matching problem and used the shape context distance to initialize the matching of graphs, followed by relaxation labeling updates. Huang [13] introduced a hierarchical shape registration algorithm using a B-spline based-Incremental Free Form Deformations (IFFD) model to recover the local registration field between two globally aligned shapes.

This chapter deals with the global registration (more specifically affine transformation) using the signed distance function which is widely used in the registration methods and shape models and point correspondence. The input shapes were implicitly embedded into a higher dimensional space of distance transforms. Then, a new dissimilarity criterion is used which is optimized to recover the transformation parameters that globally align the two input shapes. This new criterion supports both similarity and affine (without shearing) transformations. A comparison with the method presented in [1] is illustrated by a specific $2 \mathrm{D}$ example as a way of validating the proposed method.

\subsection{Shape Representation}

Human anatomical structures such as spine bones, kidneys, livers, hearts, and eyes may have similar shapes [14]. These shapes usually do not differ greatly from one individual to another. There are many works which represent and model the shape 
variability. The objective of a shape representation is to describe the desired features of the shape of interest and serve the shape descriptor to be a good classifier to differentiate among all the shapes involved [15]. Also, the shape representation significantly affect the shape registration algorithm. This section will briefly overview the shape representation and modeling techniques. In general, the shape representations methods can be folded into three categories:

(a) Landmark based,

(b) Contour (edge) based, and

(c) Region based methods.

One of the most important study for the landmark based shape representation and modeling is the active shape models (ASM) and active appearance models (AAM) proposed by Cootes et al. $[16,17,18]$. The active contour models method is a contour (edge) based method proposed by Kass et al [19]. This method is also categorized as the explicit shape representation which requires parameterizations of the contour. Also, Fourier descriptors, shape signatures, wavelet descriptors are some of the contour based shape representations. Landmark and contour based representations, which can be called as the explicit shape representation, suffer when applied to shape modeling since they do not allow the shape to undergo topological changes. Also, these representations requires point-wise correspondence between training shapes.

This work represents shapes using the regions based methods. Medial axis, convex hull, and level sets representations are some of the region based shape representations. The shape representation using the level set method [20] is known as the implicit representation 

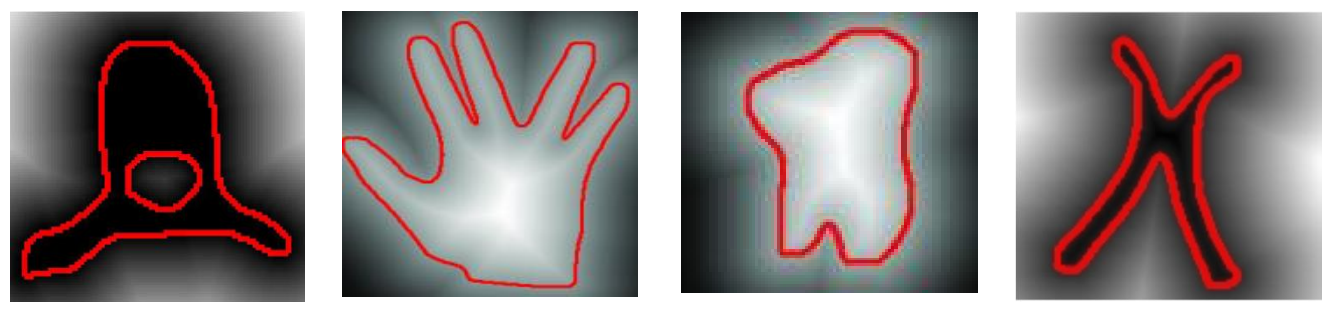

Figure 3. 1. Implicit shape representation using the signed distance function.

which does not need contour parameterizations and does handle the topological changes of shapes.

In this work, the shape is represented using the signed distance function (SDF) which is used firstly in registration by Paragios et al. [21]. Let I: $\boldsymbol{\Omega} \rightarrow \boldsymbol{R}$ be an $\boldsymbol{n}-\boldsymbol{D}$ image usually $\boldsymbol{n}=\mathbf{2}$ or $\boldsymbol{n}=\mathbf{3}, \boldsymbol{\phi}: \boldsymbol{\Omega} \rightarrow \boldsymbol{R}$ be a function that refers to a distance function representation for a given shape/contour $\boldsymbol{S}$ where $\boldsymbol{\Omega} \subset \boldsymbol{R}^{\boldsymbol{n}}$ be an image domain which is bounded. The shape can be represented as follows:

$$
\phi_{\mathcal{S}}(x, y)=\left\{\begin{array}{l}
0,(x, y) \in \mathcal{S} \\
-E D((x, y), \delta)>0,(x, y) \in \mathcal{R}_{\mathcal{S}} \\
+E D((x, y), \delta)<0,(x, y) \in \Omega-\left[\mathcal{R}_{\mathcal{S}}\right]
\end{array}\right.
$$

where $\mathcal{R}_{\boldsymbol{S}}$ represents the inside region of the shape $\boldsymbol{S}$. Let $(\boldsymbol{u}, \boldsymbol{v})$ represents an pixel location on $\boldsymbol{S}$. For $\forall(\boldsymbol{x}, \boldsymbol{y}) \in \boldsymbol{\phi}$, the distance between any $(\boldsymbol{x}, \boldsymbol{y})$ point and its nearest surface point can be calculated as follows:

$$
E D((x, y), \delta)=\min _{(u, v) \in \mathcal{S}} \sqrt{(u-x)^{2}+(v-y)^{2}}
$$

Examples of such representation are shown on Figure 3.1. 


\subsection{Basics of Shape Registration}

Registration is the important method for shape-based segmentation, shape recognition, tracking, feature extraction, image measurements, and image display. Shape registration can be defined as the process of aligning two shapes of a scene [22]. Registration requires transformations, which are mappings of points from the source (reference) shape to the target (sensed) shape [23]. The registration problem is formulated such that a transformation that moves a point from a given source shape to another target shape according to some dissimilarity measure, needs to be estimated [21]. The dissimilarity measure can be defined according to either the curve or to the entire region enclosed by the curve. Figures 3.2 shows an example of the registration process from the source to the target shape. The source and target shapes and transformation can be defined as follows:

- Source $\left(\boldsymbol{C}_{\boldsymbol{S}}\right)$ shape which is kept unchanged and is used as a reference

- Target $\left(\boldsymbol{C}_{\boldsymbol{T}}\right)$ shape which is geometrically transformed to the source shape.

- Transformation (A): The function is used to warp the target shape to take the geometry of the reference shape [22]. The transformation can be written as a function $\mathbf{A}: \boldsymbol{R}^{2} \rightarrow \boldsymbol{R}^{2}$ which is applied to a point $\mathbf{x}$ in $\boldsymbol{C}_{S}$ to produce a transformed point which is calculated as $\mathbf{X}=\mathbf{A}(\mathbf{x})$. The registration error is calculated as $\mathbf{A}(\mathbf{x})-\mathbf{y}$ for each transformed pixel. 


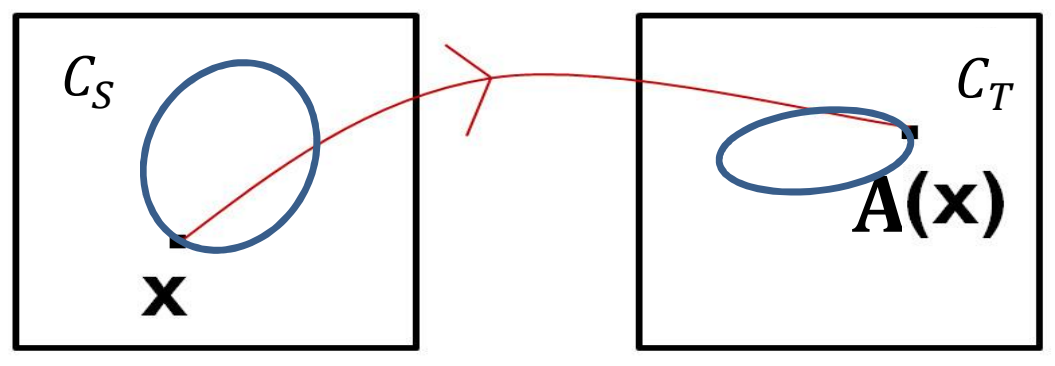

Figure 3.2. Registration example of a point from the source to the target shape.

In general, there are three categories of the registration methods: rigid, affine, and elastic transformation. In literature the rigid and affine transformations are classified as global transformations and elastic transformations are as local transformation [24]. A transformation is global if it is applied to the entire image. A transformation is local if it is a composition of two or more transformations determined on different domains (subimages) of the image.

- A rigid body transformation is the most fundamental transformation and is useful especially when correcting misalignment in the scanner. This transformation allows only translation and rotations, and preserves all lengths and angles in an image.

- An affine transformation allows translation, rotation, and scaling. Some authors defined the affine transformation as the rigid transformation plus scaling. Affine transformations involving shearing (projection) are called projective transformation. An affine transformations will map lines and planes into lines and planes but does not preserve length and angles. 
- An elastic transformation allows local translation, rotation, and scaling, and it has more number of parameters than affine transformations. It can map straight lines into curves. An elastic registration is also called as a non-linear or curved transformation. This transformation allows different regions to be transformed independently.

A global transformation is used to register $\boldsymbol{C}_{\boldsymbol{S}}$ to $\boldsymbol{C}_{\boldsymbol{T}}$ with scale, rotation, and translation parameters. For the $2 \mathrm{D}$ case, the transformation is assumed to have scaling, rotation, and translation components, as follows:

$$
\mathbf{S}=\left[\begin{array}{ll}
s_{x} & 0 \\
0 & s_{y}
\end{array}\right], \mathbf{R}=\left[\begin{array}{rr}
\cos (\theta) & -\sin (\theta) \\
\sin (\theta) & \cos (\theta)
\end{array}\right], \operatorname{Tr}=\left[\mathcal{T}_{x}, \mathcal{T}_{y}\right]^{t}
$$

The transformation will be in the form:

$$
\mathbf{A}(\mathbf{x})=\mathbf{X}=\mathbf{S R x}+\mathbf{T r}
$$

As said before, the global registration using the signed distance function is adopted which is widely used in the registration methods and shape models and point correspondence.

\subsection{Global Registration of Shapes Using the Variational approach}

This section focuses on the specific implicit representation of shapes using signed distance transforms and how this representation can be used for global shape alignment. The implicit representation of shapes using the signed distance map was employed before to achieve global alignment of shapes (e.g., [25-27]). This representation is proven to be invariant to rotations and translations, and can be efficiently used in the case of homogeneous scaling. This chapter presents a cost function which measures the disparity 
between the implicit representations of the two input shapes. This measure leads to accurate results even when dealing with anisotropic scales.

Global transformation models are usually defined by a small set of parameters. These models include, among others, the rigid transformation (translations and rotations), the similarity transformation (translations, rotations, and isotropic scaling), the affine transformation, which in addition to translations and rotations, accounts for anisotropic scaling and/or shearing. Such a transformation can be used alone to efficiently align two shapes, or it can be used as a pre-step for a local matching algorithm. Several techniques have been proposed to achieve global alignment between shapes. Some of these techniques are feature-based (e.g., Fahmi, et al., 2006[28]). Such a technique proceeds by extracting salient features and uses them to match a set of corresponding points. Finally, the matched points are used to recover the transformation parameters. Other methods, on the other hand, recover these parameters by directly optimizing a similarity/dissimilarity criterion between the two shape representations. For instance, in Huang et al., 2006 [1], an approach is proposed to achieve global registration of shapes by maximizing the $M I$ between the $S D F$ representation of the input shapes.

In [30], a new sum of squared differences (SSD) criterion is introduced in the space of signed distance transforms to globally align shapes. This new criterion can handle both rigid and affine transformations and leads to more accurate results when compared to other criteria. 


\subsubsection{SDF-based dissimilarity measure}

Let $\boldsymbol{\Phi}_{S}$ and $\boldsymbol{\Phi}_{\boldsymbol{T}}$ be the signed distance representations of the shapes, $\boldsymbol{C}_{\boldsymbol{S}}$ and $\boldsymbol{C}_{\boldsymbol{T}}$, respectively. Consider an image point $\mathbf{x}$, whose transform by $\mathbf{A}$ is denoted by $\hat{\mathbf{x}}$, and let $\widehat{\boldsymbol{\Phi}}_{\boldsymbol{S}}$ be the level set function obtained by transforming $\boldsymbol{\Phi}_{\boldsymbol{S}}$ by $\mathbf{A}$.

So that, new sum-of-squared differences criterion were introduced in order to recover the parameters of the transformation $\mathbf{A}$. We derive the formaulas for that measure for $2 \mathrm{D}$ and $3 \mathrm{D}$ cases as below.

\subsubsection{Two Dimensional Case:}

The dissimilarity measure is defined as follows:

$$
\mathcal{D}(\mathbf{S}, \mathbf{R}, \mathbf{T r})=\int_{\Omega}\left(\|\mathbf{S}\| \Phi_{S}(\mathbf{x})-\Phi_{T}(\mathbf{A x})\right)^{2} d \mathbf{x}
$$

where, $\|\mathbf{S}\|=\max \left(\left|\boldsymbol{s}_{x}\right|,\left|\boldsymbol{s}_{y}\right|\right)$ is the infinity norm of the matrix $\mathbf{S}$. Note that in the absence of scale variations, our measure coincides with the one proposed in Paragios et al., 2003[25]. For computational and technical considerations, one can consider a narrow band formed of points that are a distance $\varepsilon$ away from the source shape and their projections on the target shape,

$$
\mathcal{D}(\mathbf{S}, \mathbf{R}, \mathbf{T r})=\int_{\Omega} \delta_{\varepsilon}\left(\Phi_{S}(\mathbf{x}), \Phi_{T}(\mathbf{A x})\right)\left[\|S\| \Phi_{S}(\mathbf{x})-\Phi_{T}(\mathbf{A x})\right]^{2} d \mathbf{x}
$$

where, $\boldsymbol{\delta}_{\varepsilon}(\mathbf{a}, \mathbf{b})=\left\{\begin{array}{cc}0, & \text { if } \min (|\mathbf{a}|,|\mathbf{b}|)>\boldsymbol{\varepsilon} \\ 1, & \text { otherwise }\end{array}\right.$

Each parameter of the transformation $\mathbf{A}$ is recovered by solving its corresponding EulerLagrange equations using a gradient descent scheme[10]:

$$
\frac{d s_{x}}{d t}=2 \int_{\Omega}\left[\frac{d\|S\|}{d s_{x}} \Phi_{S}(\mathbf{x})-\nabla \Phi_{T}^{t}(A \mathbf{x})\left(\begin{array}{c}
x \cos \theta+y \sin \theta \\
0
\end{array}\right)\right] . \mathbf{r}(\mathbf{x}) d \mathbf{x}
$$




$$
\begin{aligned}
& \frac{d s_{y}}{d t}=2 \int_{\Omega}\left[\frac{d\|S\|}{d s_{y}} \Phi_{S}(\mathbf{x})-\nabla \Phi_{T}^{t}(\mathrm{Ax})\left(\begin{array}{c}
0 \\
-x \sin \theta+y \cos \theta
\end{array}\right)\right] \cdot \mathbf{r}(\mathbf{x}) d \mathbf{x}, \\
& \frac{d \theta}{d t}=2 \int_{\Omega}\left[\nabla \Phi_{T}^{t} \cdot\left(\begin{array}{l}
s_{x}(-x \sin \theta+y \cos \theta) \\
s_{y}(-x \cos \theta-y \sin \theta)
\end{array}\right)\right] \cdot \mathbf{r}(\mathbf{x}) d \mathbf{x}, \\
& \frac{d \mathcal{T}_{x}}{d t}=2 \int_{\Omega} \frac{\partial \Phi_{T}}{\partial x}(\mathrm{Ax}) \cdot \mathbf{r}(\mathbf{x}) d \mathbf{x}, \\
& \frac{d \mathcal{T}_{y}}{d t}=2 \int_{\Omega} \frac{\partial \Phi_{T}}{\partial y}(\mathrm{Ax}) \cdot \mathbf{r}(\mathbf{x}) d \mathbf{x},
\end{aligned}
$$

where $\mathbf{r}(\mathbf{x})=\boldsymbol{\delta}_{\varepsilon}\left(\boldsymbol{\Phi}_{S}(\mathbf{x}), \boldsymbol{\Phi}_{\boldsymbol{T}}(\mathbf{x})\right)\left(\|\mathbf{S}\| \boldsymbol{\Phi}_{S}(\mathbf{x})-\boldsymbol{\Phi}_{\boldsymbol{T}}(\boldsymbol{A} \mathbf{x})\right)$, and $\boldsymbol{\nabla}$ denotes the gradient operator. Note that, since positive scale values are considered, the terms $\frac{d\|S\|}{d s_{x ; y}}$ equal either 0 or 1.

\subsubsection{Three Dimensional Case}

For the three dimensional case, the similarity transform $\mathbf{A}$ is defined by:

$$
\mathbf{A x}=\mathcal{S} . \mathcal{R} . \mathbf{x}+\mathcal{T} \text { where, } \mathcal{S}=\left(\begin{array}{lll}
\boldsymbol{s}_{x} & \mathbf{0} & \mathbf{0} \\
\mathbf{0} & \boldsymbol{s}_{\boldsymbol{y}} & \mathbf{0} \\
\mathbf{0} & \mathbf{0} & \boldsymbol{s}_{z}
\end{array}\right), \mathcal{T}=\left(\begin{array}{l}
\mathcal{T}_{\boldsymbol{x}} \\
\mathcal{T}_{\boldsymbol{y}} \\
\mathcal{T}_{z}
\end{array}\right)
$$

and $\mathcal{R}=\mathcal{R}\left(\boldsymbol{\theta}_{\boldsymbol{x}}, \boldsymbol{\theta}_{\boldsymbol{y}}, \boldsymbol{\theta}_{z}\right)=\mathcal{R}_{\boldsymbol{x}} \cdot \mathcal{R}_{\boldsymbol{y}} \cdot \mathcal{R}_{z}$, with

$$
\mathcal{R}_{x}=\left(\begin{array}{lll}
1 & 0 & 0 \\
0 & \cos \theta_{x} & \sin \theta_{x} \\
0 & -\sin \theta_{x} & \cos \theta_{x}
\end{array}\right), \mathcal{R}_{y}=\left(\begin{array}{lll}
\cos \theta_{y} & 0 & -\sin \theta_{y} \\
0 & 1 & 0 \\
\sin \theta_{y} & 0 & \cos \theta_{y}
\end{array}\right), \mathcal{R}_{z}=
$$

$$
\left(\begin{array}{lll}
\cos \theta_{z} & \sin \theta_{z} & 0 \\
-\sin \theta_{y} & \cos \theta_{z} & 0 \\
0 & 0 & 1
\end{array}\right)
$$

In this case, the cost function, to be minimized in order to recover the nine parameters of the transformation $\mathbf{A}$, is given by:

$$
\mathcal{D}(\mathcal{S}, \mathcal{R}, \mathcal{T})=\int_{\Omega}\left(\|\mathcal{S}\| \Phi_{S}(\mathbf{x})-\Phi_{T}(\mathbf{A x})\right)^{2} d \mathbf{x}
$$


where, $\|\boldsymbol{S}\|=\max \left(\left|\boldsymbol{s}_{\boldsymbol{x}}\right|,\left|\boldsymbol{s}_{\boldsymbol{y}}\right|,\left|\boldsymbol{s}_{\boldsymbol{y}}\right|\right)$ is the infinity norm of the matrix $\boldsymbol{S}$.

As was done in the 2D case, a narrow band formed of points can be considered that are a distance $\varepsilon$ away from the source shape and their projections on the target shape, and solve the following Euler-Lagrange equations for each of the nine parameters of $\mathbf{A}$ using a gradient descent scheme[10]:

$$
\begin{aligned}
& \frac{d s_{x}}{d t}=2 \int_{\Omega}\left[\frac{d\|\delta\|}{d s_{x}} \Phi_{S}(\mathrm{x})-\nabla \Phi_{T}^{t}(\mathrm{Ax})\left(\begin{array}{l}
R_{1} x+R_{1} y+R_{1} z \\
0 \\
0
\end{array}\right)\right] \cdot \mathbf{r}(\mathbf{x}) d \mathbf{x}, \\
& \frac{d s_{y}}{d t}=2 \int_{\Omega}\left[\frac{d\|\delta\|}{d s_{y}} \Phi_{S}(\mathrm{x})-\nabla \Phi_{T}^{t}(\mathrm{Ax})\left(\begin{array}{l}
0 \\
\mathbf{R}_{21} \mathrm{x}+\mathrm{R}_{22} \mathrm{y}+\mathrm{R}_{23} \mathrm{z} \\
0
\end{array}\right)\right] \cdot \mathbf{r}(\mathbf{x}) d \mathbf{x}, \\
& \frac{d s_{z}}{d t}=2 \int_{\Omega}\left[\frac{d\|S\|}{d s_{z}} \Phi_{S}(\mathrm{x})-\nabla \Phi_{T}^{t}(\mathrm{Ax})\left(\begin{array}{l}
0 \\
0 \\
R_{31} x+R_{32} y+R_{33} z
\end{array}\right)\right] \cdot \mathbf{r}(\mathbf{x}) d \mathbf{x},
\end{aligned}
$$

Similarly,

$$
\begin{aligned}
\frac{d \theta_{x}}{d t} & =2 \int_{\Omega}\left[\nabla \Phi_{T}^{t}(\mathrm{Ax}) \cdot \mathcal{S} \cdot \mathcal{R}_{x}^{\prime} \cdot \mathcal{R}_{y} \cdot \mathcal{R}_{z} \cdot \mathbf{x}\right] \cdot \mathbf{r}(\mathbf{x}) d \mathbf{x} \\
\frac{d \theta_{y}}{d t} & =2 \int_{\Omega}\left[\nabla \Phi_{T}^{t}(\mathrm{Ax}) \cdot \mathcal{S} \cdot \mathcal{R}_{x} \cdot \mathcal{R}_{y}^{\prime} \cdot \mathcal{R}_{z} \cdot \mathbf{x}\right] \cdot \mathbf{r}(\mathbf{x}) d \mathbf{x} \\
\frac{d \theta_{z}}{d t} & =2 \int_{\Omega}\left[\nabla \Phi_{T}^{t}(\mathrm{Ax}) \cdot \mathcal{S} \cdot \mathcal{R}_{x} \cdot \mathcal{R}_{y} \cdot \mathcal{R}_{z}^{\prime} \cdot \mathbf{x}\right] \cdot \mathbf{r}(\mathbf{x}) d \mathbf{x} \\
\frac{d \mathcal{T}_{x}}{d t} & =2 \int_{\Omega} \frac{\partial \Phi_{T}}{\partial x}(\mathrm{Ax}) \cdot \mathbf{r}(\mathrm{x}) d \mathbf{x} \\
\frac{d \mathcal{T}_{y}}{d t} & =2 \int_{\Omega} \frac{\partial \Phi_{T}}{\partial y}(\mathrm{Ax}) \cdot \mathbf{r}(\mathrm{x}) d \mathbf{x} \\
\frac{d \mathcal{T}_{z}}{d t} & =2 \int_{\Omega} \frac{\partial \Phi_{T}}{\partial z}(\mathrm{Ax}) \cdot \mathbf{r}(\mathrm{x}) d \mathbf{x}
\end{aligned}
$$


where $\mathbf{r}(\mathbf{x})=\boldsymbol{\delta}_{\varepsilon}\left(\boldsymbol{\Phi}_{S}(\mathbf{x}), \boldsymbol{\Phi}_{T}(\mathbf{x})\right)\left(\|\boldsymbol{S}\| \boldsymbol{\Phi}_{S}(\mathbf{x})-\boldsymbol{\Phi}_{\boldsymbol{T}}(\mathbf{A x})\right), \quad \boldsymbol{\nabla}$ denotes the gradient operator, and

$$
\begin{aligned}
\mathcal{R}_{x}^{\prime}= & \left(\begin{array}{lll}
0 & 0 & 0 \\
0 & -\sin \theta_{x} & \cos \theta_{x} \\
0 & -\cos \theta_{x} & -\sin \theta_{x}
\end{array}\right), \mathcal{R}_{y}^{\prime}=\left(\begin{array}{lll}
-\sin \theta_{y} & 0 & -\cos \theta_{y} \\
0 & 0 & 0 \\
\cos \theta_{y} & 0 & -\sin \theta_{y}
\end{array}\right) \\
\mathcal{R}_{\mathrm{z}}^{\prime} & =\left(\begin{array}{lll}
-\sin \theta_{\mathrm{z}} & \cos \theta_{\mathrm{z}} & 0 \\
-\cos \theta_{\mathrm{y}} & -\sin \theta_{\mathrm{z}} & 0 \\
0 & 0 & 0
\end{array}\right) .
\end{aligned}
$$

Note that, since we are considering positive scale values, the terms $\frac{d\|\mathcal{S}\|}{\boldsymbol{d} \boldsymbol{s}_{\boldsymbol{x} ; \boldsymbol{y} ; \boldsymbol{z}}}$ equal either 0 or 1

\subsubsection{Experimental results}

To quantitatively validate the model in (3.6), several registration experiments were performed. For each trial, the source shape is fixed and the target shape is generated by deforming the source using a known transformation $\mathbf{A}=\mathbf{A}(\mathbf{S}, \mathbf{R}, \mathbf{T r})$ which will be considered as the Ground Truth $(G T)$. Then, this model is used to recover the optimal alignment parameters. The recovered parameters are then compared to the $G T$ and to those obtained when using a homogeneous scale-based measure; the case in which, $\boldsymbol{s}_{\boldsymbol{x}}=\boldsymbol{s}_{\boldsymbol{y}}=$ $\boldsymbol{S}$, the matrix $\boldsymbol{S}$ reduces to the scalar $\boldsymbol{S}$, and the measure given by (3.6) is changed accordingly, as well as the Euler-Lagrange equations, as presented in [25]. In each case, the algorithm leads to more accurate results and one can see that the isotropic scale-based criterion completely fails when the difference between $\boldsymbol{s}_{\boldsymbol{x}}$ and $\boldsymbol{s}_{\boldsymbol{y}}$ is large (see e.g., last two columns of Figure 3.3). Table 3.1 summarizes some of these results.

Another set of experiments was carried out to compare the registration performance of the proposed criterion with the other two criteria (as in [25] and [26]) by registering 
pairs of arbitrary shapes that belong to the same class. Some of these results are presented on Figure 3.4 and Figure 3.5 respectively. One can easily notice from these figures that the proposed method outperforms the other two methods.

Table 3.1. Comparison of recovered parameters when using our model (M1) (Equation. 2.6) vs. the use of similarity-based model (M2), i.e., $\mathbf{s}_{\mathbf{x}}=\mathbf{s}_{\mathbf{y}}=\mathbf{s}$, (Paragios et al., 2003[25]); GT stands for ground truth.

\begin{tabular}{|c|c|c|c|c|c|c|}
\hline \multirow{2}{*}{ Transformation } & \multicolumn{3}{|c|}{ Corpus Callosum } & \multicolumn{3}{c|}{ Fish } \\
\cline { 2 - 7 } & GT & M1 & M2 & GT & M1 & M2 \\
\hline $\mathbf{s}_{\mathbf{x}}$ & 1.5 & 1.50 & 0.99 & 0.6 & 0.60 & 0.71 \\
\hline $\mathbf{s}_{\mathbf{y}}$ & 0.9 & 0.90 & - & 1.0 & 1.00 & - \\
\hline $\boldsymbol{\theta}^{\circ}$ & 10 & 10 & 22.75 & 60 & 60 & 52.03 \\
\hline $\boldsymbol{T}_{\boldsymbol{x}}$ & 2.5 & 1.61 & 0.14 & -3.5 & -4.17 & -5.82 \\
\hline $\boldsymbol{T}_{\boldsymbol{y}}$ & 0.0 & -0.6 & 2.50 & -5 & -5.16 & -7.52 \\
\hline
\end{tabular}

Finally, several three dimensional experiments were carried out to test the proposed global alignment algorithm. A 3D tooth shape of size $11 \times 1 \times 1 \times 1$ 25is used as te target shape. This shape is used to generate various deformed instances by randomly assigning different values to the transformation parameters $\left(\boldsymbol{s}_{\boldsymbol{x}}, \boldsymbol{s}_{\boldsymbol{y}}, \boldsymbol{s}_{\boldsymbol{z}}, \boldsymbol{\theta}_{\boldsymbol{x}}, \boldsymbol{\theta}_{\boldsymbol{y}}, \boldsymbol{\theta}_{z}, \boldsymbol{t}_{\boldsymbol{x}}, \boldsymbol{t}_{\boldsymbol{y}}\right.$, and $\left.\boldsymbol{t}_{\mathbf{z}}\right)$. For each trial, the generated deformed shape is used as the source and is registered to the target shape using the proposed algorithm. Some of the corresponding results are shown on Figure 3.6. For each trial, one can notice the high accuracy of the registration results. 


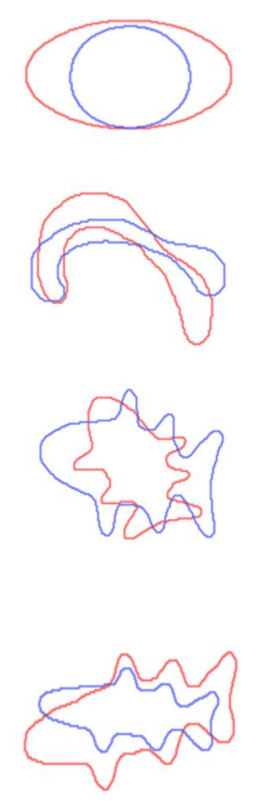

(a)
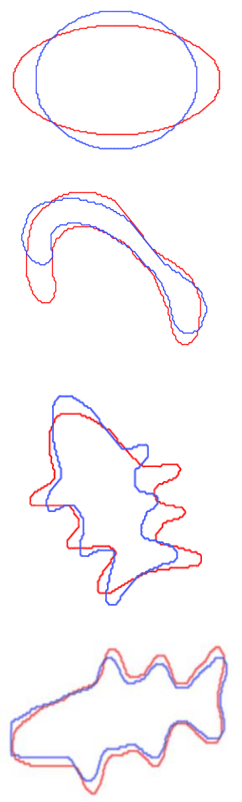

(b)
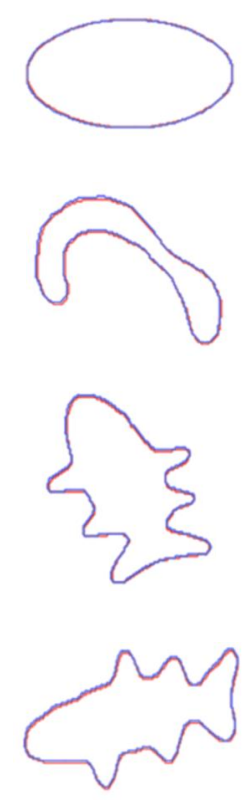

(c)

Figure 3.3. Global registration. (a) Input Shapes (blue: source; red: target). (b) Registration results using the isotropic-scale based model, (c) Registration using the proposed model (2.6).
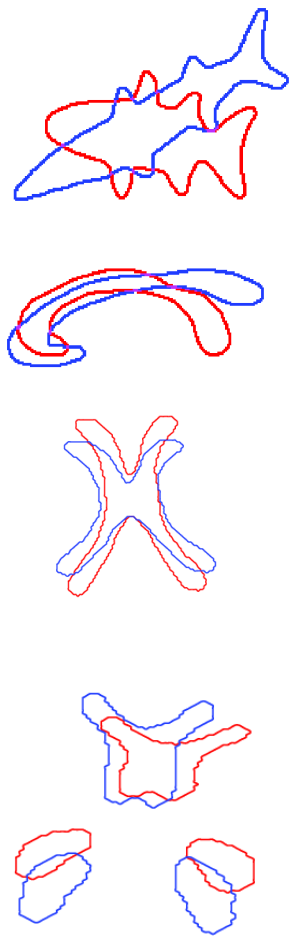

(a)
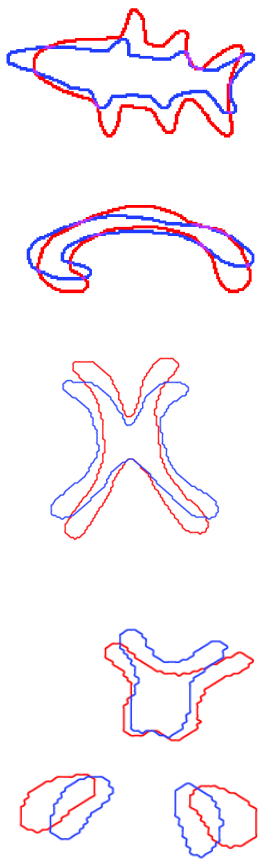

(b)
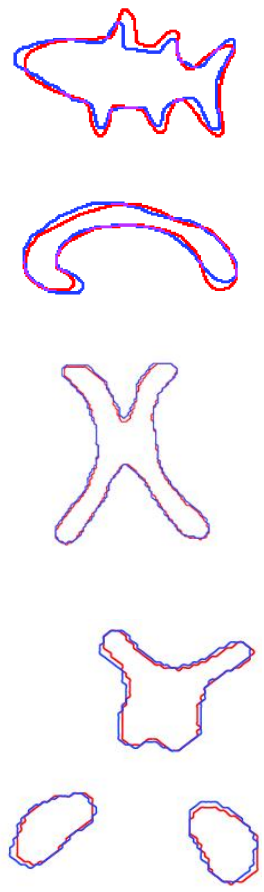

(c)

Figure 3.4. Global registration. (a) Input Shapes. (b) Registration using homogeneous scale-based measure, (c) Registration using the proposed registration model (2.6). 

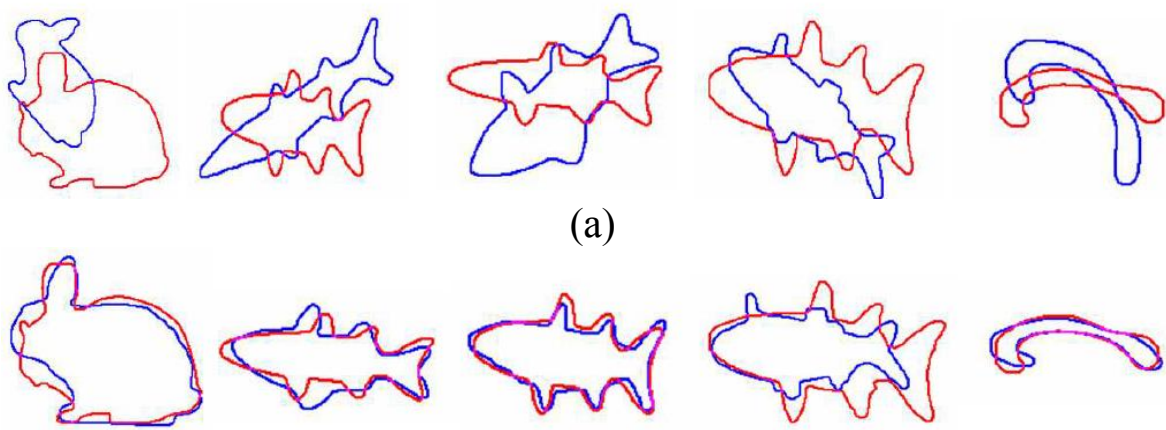

(b)
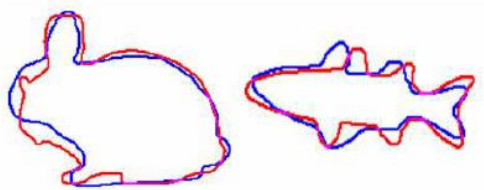
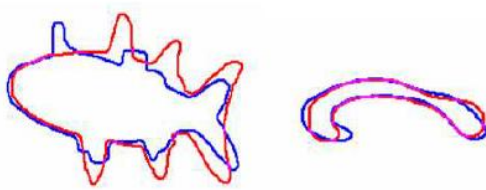

(c)

Figure 3.5. Global Matching: Proposed Signed Distance- vs. VDF-based affine registrations [26]. (a) Input Shapes. (b) Affine Registration using the VDF representation (c) Affine Registration using the new SSD criterion (2.6).

(a)
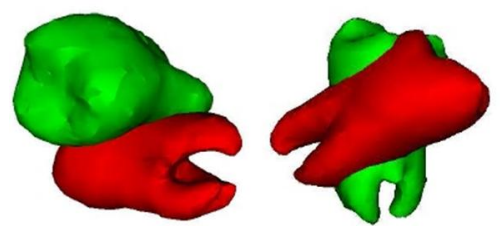

(b)

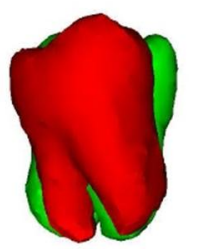

(c)

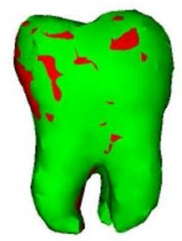

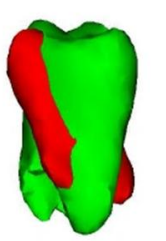

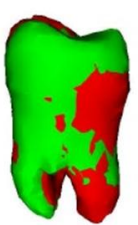

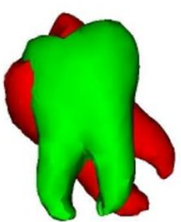
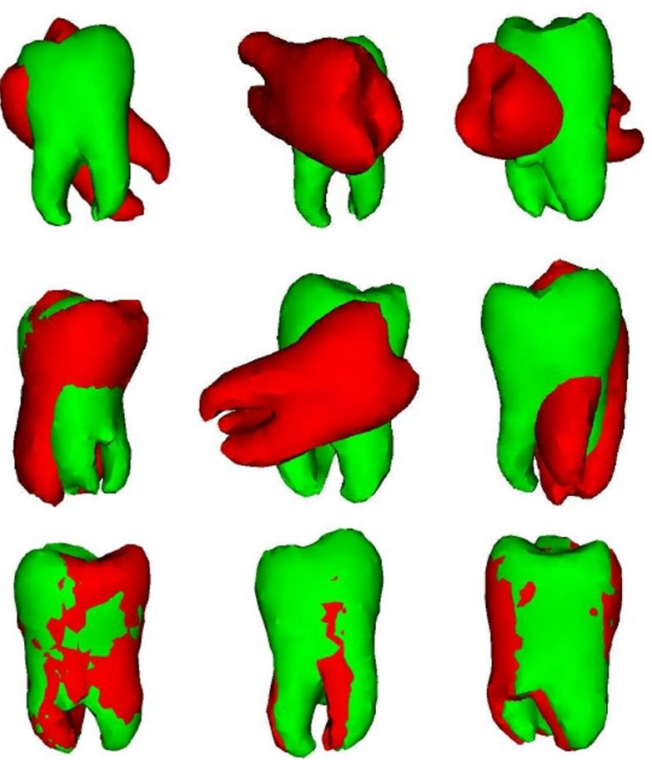

Figure 3.6. Global registration of 3D shapes of two tooth shapes. (a) Input Shapes. (b) An intermediate state. (c) Registration using the proposed registration model (2.10). 
(a)

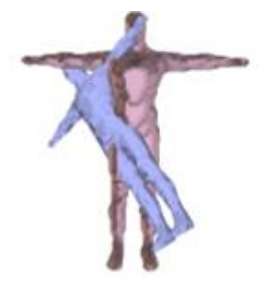

(b)

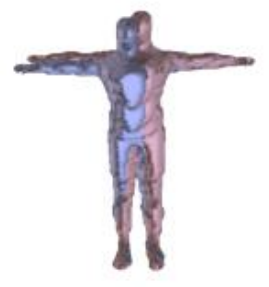

(c)

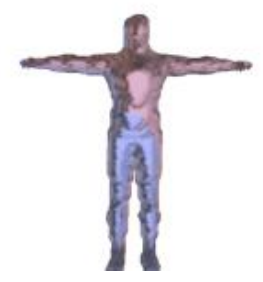

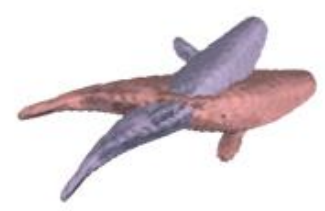
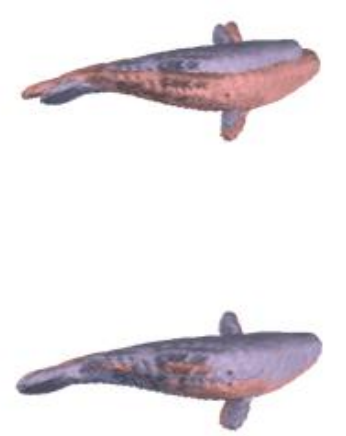
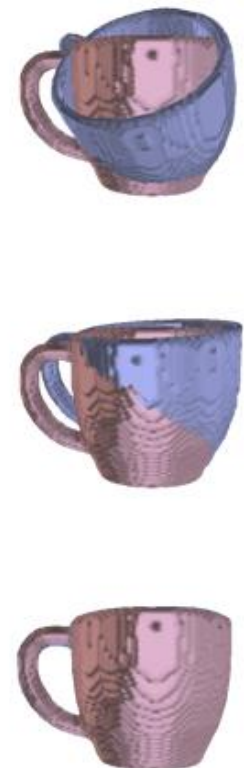

Figure 3.7. More examples for global registration of 3D shapes of various shapes. (a) Input Shapes. (b) An intermediate state. (c) Registration using the proposed registration model (2.10).

\subsection{Summary}

In this chapter, a Variational framework for global registration of shapes has been presented. The shapes are implicitly represented through their sign distance maps. A new criterion, measuring the disparity between the two representations, was proposed to globally align the input shapes. This criterion supports both rigid/similarity and affine transformations. Various experiments were presented to show the effectiveness of the proposed framework. A possible direction of the present work is its extension to image/volume registration. 


\section{CHAPTER 4}

\section{FUNDAMENTALS OF IMAGE REGISTRATION}

Image registration is establishment of correspondence between images of the same scene. Many image processing applications like remote sensing for change detection, estimation of wind speed and direction for weather forecasting, fusion of medical images like PETMRI, CT-PET etc. need image registration. Image registration is a process of aligning two images acquired by same/different sensors, at different times or from different viewpoint. To register images, it is needed to determine geometric transformation that aligns images with respect to the reference image. The most common transformations are rigid, affine, projective, perspective and global. Over the years, a large range of techniques has been developed for various types of problems. A typical image registration algorithm consists of three coupled components: an alignment measure (also known as similarity/dissimilarity measure, registration function, etc.) that quantifies the quality of alignment; a class of admissible geometric transformations that can be applied to the image(s), i.e., employed to spatially "warp" the image(s); and an optimizer that seeks the transformation that maximizes the similarity as quantified by the alignment measure. Figure 4.1 illustrates these components.

From the application's point of view, registration algorithms can be classified based on several criteria. The classifications presented here are partially based on [31]. The criteria and their primary subdivisions are:

- Modality: Mono-modal refers to the case where all images are obtained from the 


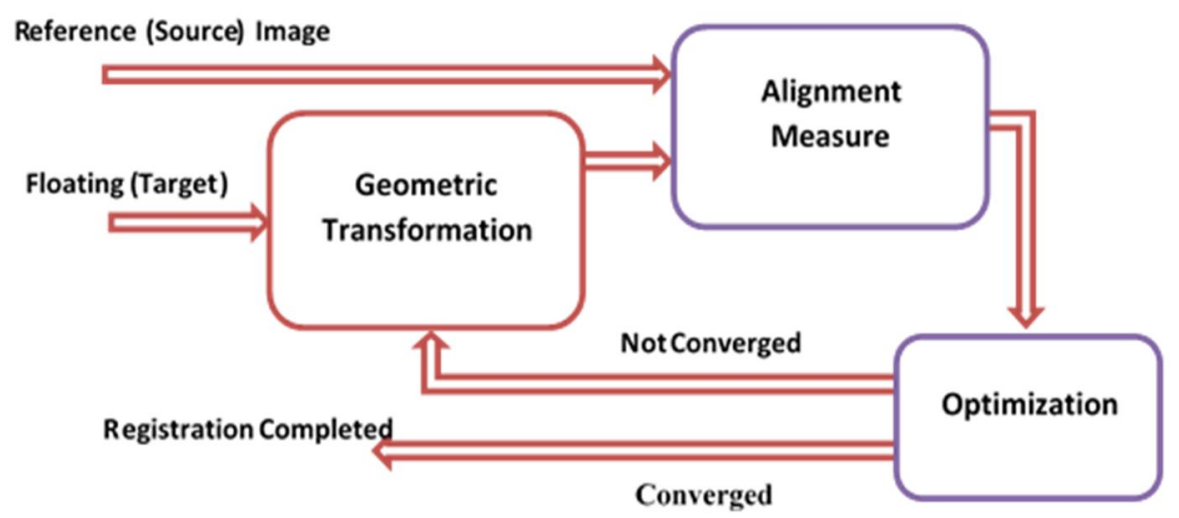

Figure4.1. Block diagram of a typical image registration algorithm.

same imaging sensor type and there are no major differences between the intensity ranges that correspond to the same physical/physiological phenomenon. In a multimodal setting, these ranges can differ drastically. This is typically due to different sensor types.

- Dimensionality: This refers to the number of dimensions of the images. Historically, images have typically had two spatial dimensions. Today, however, several imaging technologies provide 3D volumes. Moreover, some sensors, e.g. functional MRI, provide a video, i.e. a sequence of images. When treating the video as one big data set, time can be thought of as an extra dimension. One convention is to denote time as a 0.5 dimension. This is helpful to clarify some ambiguities, e.g. 3D (three spatial dimensions) versus 2.5D (two spatial dimensions + time). Most of today's applications involve 2D/2D, 3D/3D and 2D/3D registration.

- Speed: Offline refers to applications where time is not an important constraint. Online denotes a heavy time constraint, typically indicating real-time applications. An important online example is intra-operative procedures performed within the 
operating room. Some scientific applications (e.g. human brain mapping), on the other hand, do not have a heavy time constraint.

- Employed Information Content: In the registration literature, one can identify two trends in the type of information employed. Landmark based approaches rely on the definition of landmarks. Alignment is computed based on these landmarks (sets of points, lines or surfaces) only. These landmarks can have a clear physical meaning (e.g. the cortical surface of the human brain [32], fiducial markers visible in all modalities [33], etc.). In landmark based registration, the set of identified points is sparse compared to the original image content, which allows fast optimization. However, performance of the algorithm heavily depends on the landmark identification. Image content based approaches, on the other hand, rely on pixel intensity information. These typically extract features from pixels (e.g. intensity values [34], gradient vectors [35], wavelet coefficients [36], etc.) and compute an alignment based on the set of feature samples. These are usually slower than landmark based algorithms, but have the potential to produce accurate and robust results in contexts where landmarks are difficult to define or determine.

- Locality of Alignment Measure: Alignment quality can be measured for the whole image, using global measures, e.g. sum of squared differences of all pixel values, or for a neighborhood of a pixel location using local measures, e.g. local correlation.

- Transformation: Generally speaking, there are two types of geometric transformations: global models, e.g. rigid-body, affine, spline based, etc., where a small set of parameters determine the transformation and nonparametric models (also 
known as optical flow, dense matching, etc.), where each pixel is allowed to move independently. Note that in the latter case, if there was no restriction on the transformation, an image could be made to look similar to any other image with the same intensity range as the first image. Thus, these methods require regularization to overcome ill-posedness and incorporate prior knowledge about the deformation field.

- Optimization: Typically, iterative methods are employed within a multi-resolution pyramid, to speed up convergence. Popular choices of optimizers are: gradientdescent and its variants [34], Powell's method [37], Downhill simplex method and Levenberg Marquardt optimization [38].

In this chapter, our analysis are restricted to global image content-based registration approaches, which provide a general framework and require minimal knowledge about the specifics of the application domain. In the following sections, the theoretical aspects of an image registration problem are reviewed.

\subsection{Problem Definition}

Let $I_{\mathrm{j}}$ be in a family $U$ of scalar valued images defined on $\Omega$, a finite subset of $\mathrm{R}^{d}$, $d \in \mathrm{Z}+$. For example, all brain MRI volumes may constitute a family, $U$. The relationship between any two images $I_{1}$ and $I_{2}$ can be written as:

$$
I_{1}=f\left(I_{2} \circ \mathrm{A}\right)+\aleph
$$

Where $\mathrm{A}: \mathrm{R}^{d} \rightarrow \mathrm{R}^{d}$ is a geometric transformation that models the misalignment that we want to recover, $f$ is a cross-image family mapping that captures the volumetric variation and $\boldsymbol{N}$ is some noise. In this model $\left(\boldsymbol{I}_{\mathbf{1}}, \boldsymbol{I}_{\mathbf{2}} \circ \mathbf{A}\right)$ is a (optimally) registered pair of images. 
The goal of the algorithm is to estimate $\mathbf{A}$, by maximizing a similarity measure (or, minimizing a dissimilarity measure). As discussed before, we are searching for the best alignment of two images by transforming one onto the other, it is a very crucial issue to assess how similar two images actually are. The two main classes of similarity measures are feature-based and intensity-based. The following section gives more details about the intensity based measures.

\subsection{Intensity-Based Similarity Measures}

Feature-based measures do some processing with the images first in order to obtain significant information, which can be used to judge the similarity. This can be the position of significant landmarks, or the parameterization of certain shapes within the images, which are obtained by segmentation. However, this processing usually needs some user interaction, which is often not desirable.

On the contrary, intensity-based measures use the full raw image information. A similarity measure is derived using all intensity values in the two images. However, one may consider introducing a region of interest in order to omit non relevant image parts. Working with this kind of measure is often referred to as pixel based registration, too. The main advantage is that registration can be executed right after image acquisition. No further user input is needed for instance for selecting landmarks or setting the parameters for a segmentation. This is the kind of similarity measurement that we are going to use. Figure 4.2 shows an overview about the three classes of measures. 


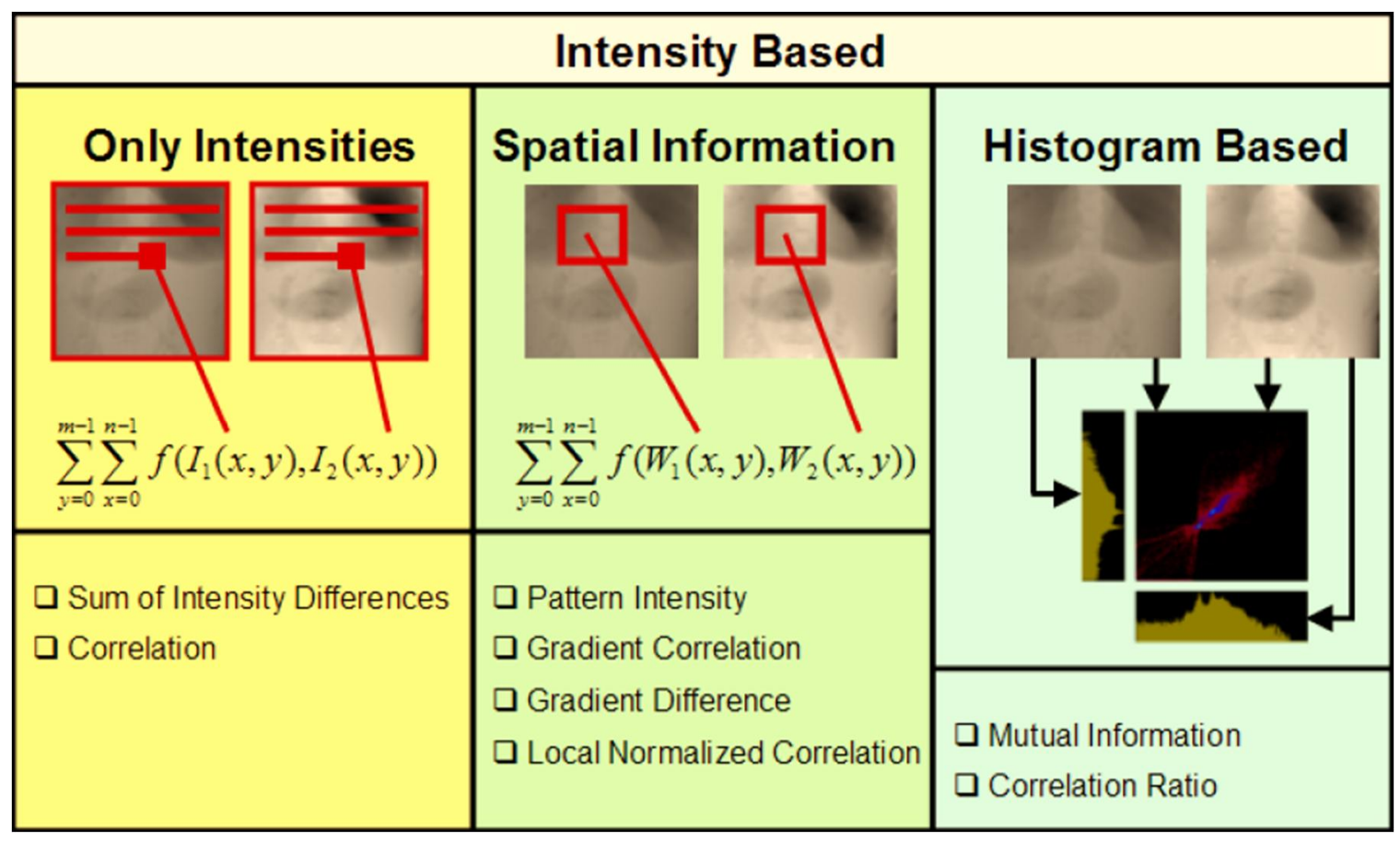

Figure4.2. Classification of image similarity measures.

\subsubsection{Measures using only Image Intensities}

This class of similarity measures compares the intensity values of both images pairwise at the same pixel positions. Subsequently one single value is composed out of it with a certain scheme. An advantage of this kind of measure is that it can be used not only with two-dimensional images, but with any kind of data in arbitrary dimensions, as no spatial information is considered.

\subsubsection{Sum of Intensity Differences}

One very simple similarity measure is the sum of squared differences (SSD) between two images:

$$
S S D=\frac{1}{N} \sum_{\mathrm{x} \in D_{\mathrm{x}}}\left(I_{1}(\mathrm{x})-I_{2}(\mathrm{x})\right)^{2}
$$


where $\mathbf{x}$ stands for the coordinates vector of the image, the vector $\mathbf{x}$ is defined on the set $D_{\mathrm{x}}$ defined as the overlap domain (eventually combined with a region of interest) of the images. $N$ is the number of pixels in $D_{\mathrm{x}}$. It can be shown that SSD is the optimum measure when two images only differ by Gaussian noise (i.e. $f$ in (4.1) to be the identity function). Therefore this measure is mostly used in registration applications where two images from the same modality are used, otherwise this constraint is not realistic. Besides simplicity, another reason to use this measure may be that specific optimization algorithms can be used, that can minimize sum-of-squares expressions very efficiently. Due to its simplicity, this measure is especially used for intramodality registration [39].

\subsubsection{Correlation Coefficient}

Linear correlation is used everywhere in mathematical and computer science problems, when the relation between two data sets have to be assessed in a fast and simple way. In this case, the linear dependency of the pixel values in one image to the other is needed to be quantized. This measure is called Normalized Cross Correlation (NCC), often referred to as Correlation Coefficient as well:

$$
N C C=\frac{\sum_{\mathrm{x} \in D_{\mathrm{x}}}\left(I_{1}(\mathrm{x})-\overline{I_{1}}\right) \cdot\left(I_{2}(\mathrm{x})-\bar{I}_{2}\right)}{\sqrt{\left(I_{1}(\mathrm{x})-\overline{I_{1}}\right)^{2}} \cdot \sqrt{\left(I_{2}(\mathrm{x})-\overline{I_{2}}\right)^{2}}}
$$

$\overline{I_{1}}$ and $\overline{I_{2}}$ are the mean intensity values in the images $\boldsymbol{I}_{\mathbf{1}}$ and $\boldsymbol{I}_{2}$, respectively. The denominator is the product of the standard deviations in the two images.

Normalized Cross Correlation has been used for various registration problems. In 3D-3D registration it is mainly limited to images of the same modality, however there are 
approaches using NCC on image data transformed to Fourier-space [39]. It is being used and evaluated for 2D-3D registration [40, 41] and slice-to-volume registration [42] as well.

\subsubsection{Measures using Spatial Information}

This class of measures still evaluates the data pixel-wise, but in addition some kind of neighborhood information is involved at every position. This can be done by adding all differences within a certain radius (Pattern Intensity), or by calculating gradient images for further examination.

\subsubsection{Pattern Intensity}

This measure [41] directly assesses the content of a difference image, $I_{d i f f}=I_{1}-s I_{2}$. If the images are perfectly aligned, the difference image should contain the fewest amount of patterns, or be constant in the optimum case. Pattern Intensity now sums up over differences between neighbored pixels in $I_{\text {diff }}$ :

$$
P I_{r, \sigma}(s)=\frac{1}{N} \sum_{x, y \in D_{\mathrm{x}}} \sum_{u, w} \frac{\sigma^{2}}{\sigma^{2}+\left(I_{d i f f}(x, y)-I_{d i f f}(u, w)\right)^{2}}
$$

where $(u, w) \in \boldsymbol{D}_{\mathbf{x}}$ such that $(x-u)^{2}+(y-w)^{2}<r^{2}$. Good working parameters are $r=3$ and $\sigma=10[40]$, the latter selecting the values in the difference image where the measure is the most sensitive. The scaling factor $s$ for creation of the difference image should be chosen so that the difference image has the least contrast. Note that a constant shift between the image intensities does not affect the similarity measure, as it only assesses differences in the difference image.

\subsubsection{Gradient Correlation}

By using horizontal and vertical Sobel templates, four gradient images $d I_{1} / d x, d I_{1} / d y, d I_{2} / d x$ and $d I_{2} / d y$ are created. Then Normalized Cross Correlation (4.3) is calculated of these 
horizontal and vertical gradient images, respectively. The final value of the measure is the average of the two NCCs [40].

\subsubsection{Gradient Difference}

This measure, proposed in [40] evaluates two difference images $I_{\text {diff } \mathrm{H}}$ and $I_{\text {diff } \mathrm{V}}$ which are

calculated from gradient images. Same $1 /\left(1+x^{2}\right)$ structure as pattern intensity is applied:

$$
\begin{gathered}
G D(s)=\sum_{x, y \in D_{\mathrm{x}}} \frac{A_{h}}{A_{h}+\left(I_{d i f f \mathrm{H}}(x, y)\right)^{2}}+\sum_{x, y \in D_{\mathrm{x}}} \frac{A_{v}}{A_{v}+\left(I_{d i f f \mathrm{~V}}(x, y)\right)^{2}} \\
I_{\text {diff } \mathrm{H}}(x, y)=\frac{d I_{1}}{d x}-s \frac{d I_{2}}{d x} \text { and } I_{d i f f \mathrm{~V}}(x, y)=\frac{d I_{1}}{d y}-s \frac{d I_{2}}{d y}
\end{gathered}
$$

$A_{h}$ and $A_{v}$ are constants which work well if they are set to the variance of the respective reference image.

\subsubsection{Histogram Based Measures}

Those measures overlap with what is often termed Information Theoretic Measures. Here, successful registration means to maximize the amount of shared information in the two images, or two minimize the amount of information present in a difference image. In order to automate this idea, some measure of information is needed as prerequisite. The most commonly used one is the Shannon entropy:

$$
H=-\sum_{a} p(a) \log p(a)
$$

where $\boldsymbol{p}(\boldsymbol{a})$ denotes a discrete probability distribution. Another entropy concept adopted for registration has been introduced in [43], the Cumulative Residual Entropy. If the amount of information in an image is to be assessed, the image pixel intensity is treated as 
random variable. $\boldsymbol{p}(\boldsymbol{a})$ is then the probability that any pixel in the image has the intensity a. It is being summed up over all possible intensities, e.g. [0 . 255] for 8 bit images.
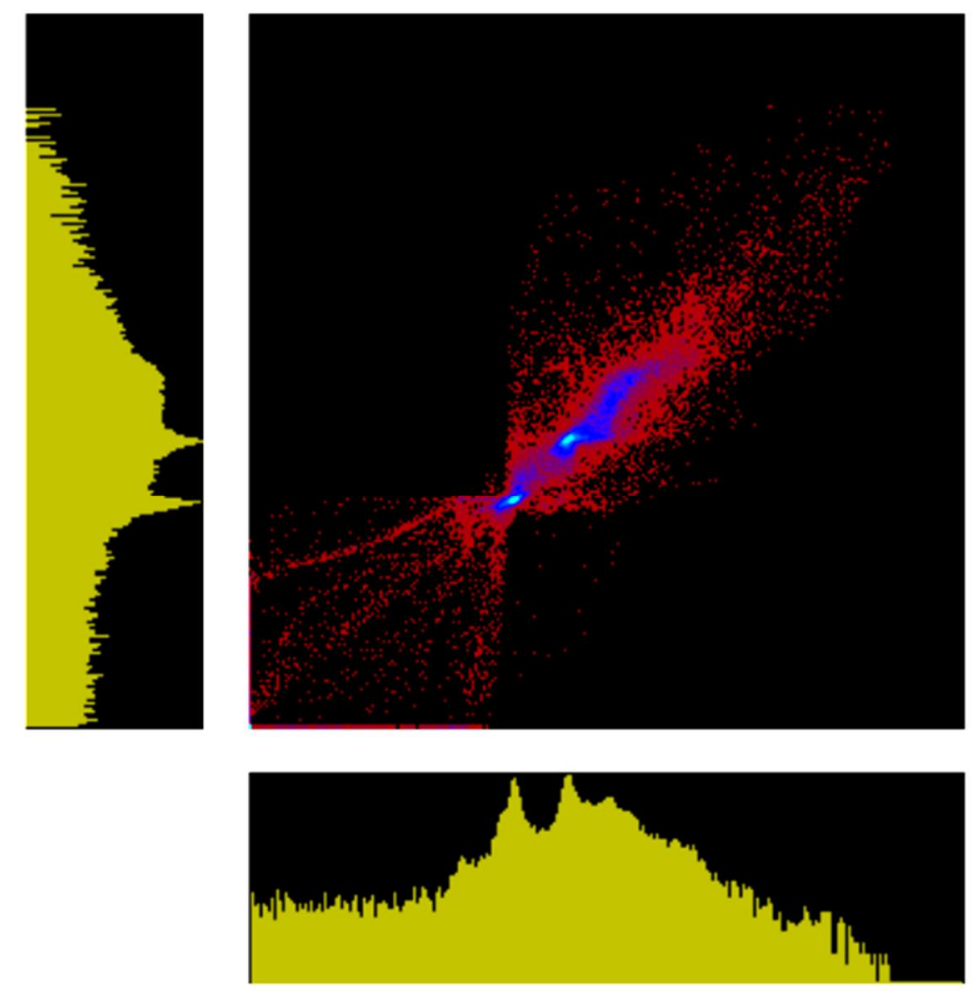

Figure4.3. Joint and individual histograms for a synthetic and an X-Ray image.

Therefore the distribution can be composed by creating a histogram (counting the occurrences of every possible intensity value) and dividing by the number of pixels in the image. Similar to that, a joint probability distribution is created by counting the occurrences of every pair of intensities in two images (at the same pixel position, respectively), resulting in a two-dimensional histogram. Figure4.3 shows a joint histogram and the respective two individual histograms. Once those distributions are computed, every further processing is done on them, without considering any of the original image data. To mention is, that the measures from section 4.2.1 can be rewritten in order to be computed based on histogram 
information, too. However, this is not the original idea of those measures, and the respective calculation is more costly.

\subsubsection{Entropy of the difference image}

In this case the entropy of a difference image $I_{\text {diff }}=I_{1}-s I_{2}$ is examined [40]. If the two images are matching perfectly, the difference image should be empty (i.e. have constant intensity values), which results in an entropy of zero.

\subsubsection{Mutual Information}

For measuring the amount of combined information in two images, the joint entropy is used:

$$
H\left(I_{1}, I_{2}\right)=\sum_{a, b} p(a, b) \log p(a, b)
$$

it is being summed up over the intensity range of both images. The intensity probabilities $p(a, b)$, also called probability distribution function (PDF) can be visualized as a twodimensional joint histogram. Every value of $p(a, b)$ tells the occurrence of the intensity $a$ in the first and intensity $b$ in the second image at the same positions, respectively. If $I_{1}$ and $I_{2}$ are totally unrelated, the joint entropy will be the sum of the entropies of the individual images. The more similar the images are, the lower the joint entropy is (if the images are the same, it equals the entropy of the images, $\left.H\left(I_{1}, I_{2}\right)=H\left(I_{1}\right)=H\left(I_{2}\right)\right)$. Mutual Information now combines the entropy calculations of the individual images and the combination:

$$
M I=H\left(I_{1}\right)+H\left(I_{2}\right)-H\left(I_{1}, I_{2}\right)=\sum_{a, b} p(a, b) \frac{\log p(a, b)}{p_{1}(a) p_{2}(b)}
$$

The range of this measure is now $\left[0, H_{\max }\right]$, where $H_{\max }$ is the maximum entropy that is possible. It can be achieved by assuming equal distribution of all intensity values in the image, i.e. a constant histogram: 


$$
H_{\text {max }}=-\sum_{a} \frac{1}{n} \log \frac{1}{n}=\log (n)
$$

where $n$ is the number of histogram entries, e.g. 256. It is desirable to normalize the mutual information measure to have a value between 0 and 1 . This can be done easily:

$$
N M I=\frac{2 M I}{H\left(I_{1}\right)+H\left(I_{2}\right)}=2-\frac{H\left(I_{1}, I_{2}\right)}{H\left(I_{1}\right)+H\left(I_{2}\right)}
$$

Some important features of normalized mutual information:

- The result is zero if one or both of the images are constant.

- The result is one if both images are identical or the pixel values in one image are only scaled and shifted with respect to the pixel values in the other image. However, this is only valid if no rounding errors occur and no intensities get lost on the bounds. This implicates that the entropies of both images are still the same.

- Varying the size of the histograms and the joint probability distribution has an important effect on the MI value. On the other hand, if high-resolution images are used and the registration estimation is already very close to the optimum, full histogram resolution results in a very sharp rise to a value of one.

A very important property of Mutual Information is, that it assumes no functional dependency between the images at all, only statistical dependence between the intensities. Due to this fact this measure is very popular in registration problems where different modalities are involved. A very comprehensive work about Mutual Information and its information-theoretic background can be found in [46].

One drawback of Mutual Information that is criticized sometimes, is that it does not consider any spatial information. In recent years some ideas have come up that would compute Mutual Information not just on intensity values, but on vectors incorporating 
intensity and one to three spatial parameters, for instance the pixel location in the images. The consequence is that the entropies of multi-dimensional probability distributions have to be computed. Those distributions, and especially the respective joint probability distribution, usually cannot be saved in an array anymore. Thus the problem is generalized to estimating the necessary entropies, discarding any explicit form of the underlying probability distributions.

\subsubsection{Correlation Ratio}

This is an alternative approach of an information theory based similarity measure, introduced by Roche et al. [47]. It can be seen as a measure of how well one image explains the other. Therefore the image intensities are treated as random variables and a functional dependency between the images is assumed. A very important characteristic of correlation ratio is, that it is not symmetric. One has to decide in advance which image should be the model, and therefore is used as a base for the estimation of the second image. The basic equation is:

$$
\rho\left(I_{2} \mid I_{1}\right)=\frac{\operatorname{Var}\left(E\left(I_{2} \mid I_{1}\right)\right)}{\operatorname{Var}\left(I_{2}\right)}
$$

In this case the variables $I_{1}$ and $I_{2}$ stand for random variables of the image intensities, respectively. Assuming that $I_{2}$ is totally independent of $I_{1}$, the expectation $E\left(I_{2} \mid I_{1}\right)=E\left(I_{2}\right)$ is constant and thus its variance is zero. On the other hand, assuming full functional dependence, every value of $I_{2}$ can be predicted given knowledge of $I_{1}$ and therefore $E\left(I_{2} \mid\right.$ $\left.I_{1}\right)=I_{2}$, resulting in a similarity measure value of 1 . Computing the actual value is done with: 


$$
\begin{gathered}
\rho\left(I_{2} \mid I_{1}\right)=1-\frac{1}{\sigma^{2}} \sum_{a} \sigma_{a}^{2} p_{1}(a) \\
\sigma^{2}=\left(\sum_{b} b^{2} p_{2}(b)\right)-\left(\sum_{b} b p_{2}(b)\right)^{2} \\
\sigma_{a}^{2}=\left(\frac{1}{p_{1}(a)} \sum_{b} b^{2} p(a, b)\right)-\left(\frac{1}{p_{1}(a)} \sum_{b} b p(a, b)\right)^{2}
\end{gathered}
$$

Correlation Ratio originates as an extension of Normalized Cross Correlation. When using this measure with images of the same modality, the behavior of Correlation Ratio and Correlation Coefficient is indeed very similar. In contrast to Mutual Information, the Correlation Ratio is not based on entropies, but on the variance of an image random variable that is conditioned with the random variable of another image. The main advantage of this is that proximity information in the intensity space is considered, whereby the entropy and joint entropy calculations only assess pairs of intensity ranges.

\subsubsection{Proposed Similarity measures}

In this framework, two novel image similarity measures are adopted from [48] and [49]. The first one is called exponential correlation (EC). The other is called pixel-based individual entropy correlation coefficient (IECC). Both are used as the similarity measure between the synthetic X-ray images and the reference X-ray image in order to evaluate the current quality of alignment. Section 4.5 explains the experiments, and evaluates the accuracy of the results.

\subsubsection{Exponential Correlation (EC)}

In this section, a novel image similarity measure is presented. It is called Exponential Correlation $(E C)$. This measure is compared to others such as $S S D$, correlation, $N C C$, joint entropy, $M I$, and $N M I$. The experiments have shown that the proposed similarity measure 
is capable of describing complex relationship between image intensity values while offering a favorable speed-performance trade-off as compared to other known similarity measures.

Given that the reference image $(R)$ and the floating image $(F)$, their $E C$ value can be calculated using the following equation:

$$
E C(R, F)=\sum_{\mathrm{x} \in D_{\mathrm{x}}}\left(\mathrm{e}^{R(\mathrm{x})-\bar{R}}-\mathbf{1}\right)\left(e^{F(\mathrm{x})-\overline{\mathrm{F}}}-\mathbf{1}\right),
$$

where $\overline{\boldsymbol{F}}$ and $\overline{\boldsymbol{R}}$ represent the mean of intensity values of images $F$ and $R$ respectively. When two images are geometrically aligned, EC value is maximized.

It is clear that the EC similarity measure (4.13) is able to determine the correspondence among images with complex relationships between the pixel values much better than NCC.

\subsubsection{Individual Entropy Correlation Coefficient (IECC)}

In this type of similarity measures, we deal with the images $R$ and $F$ as two random variables. A 1D histogram is constructed for each image. It shows the distribution of the pixel values. Since these values vary over a wide range, they were rescaled into $N=64$ bins. A 2D histogram $h(a, b)$ is obtained from the pair of floating image and reference image. Each entry in this histogram represents the number of times intensity $a$ in image $R$ coincides with intensity $b$ in the other image $F$. The probability distribution of this 2D histogram values is obtained from $h(a, b)$. It is called the joint probability distribution and can be expressed as:

$$
\boldsymbol{p}\left(\boldsymbol{a}_{i}, \boldsymbol{b}_{j}\right)=\frac{\boldsymbol{h}\left(\boldsymbol{a}_{i}, \boldsymbol{b}_{j}\right)}{\sum_{i=1}^{N} \sum_{j=1}^{N} h\left(\boldsymbol{a}_{i}, b_{j}\right)} .
$$


As all histogram based measure (Section 4.2.3), the pixel-based IECC depends on $\boldsymbol{p}\left(\boldsymbol{a}_{\boldsymbol{i}}, \boldsymbol{b}_{\boldsymbol{j}}\right)$. It represents the ratio between the pixel-based component of the mutual information between the two images, and the sum of the pixel-based components of the two marginal entropies of each image. So, IECC is expressed as [49]:

$$
\operatorname{IECC}(R, F)=\sum_{i=1}^{N} \sum_{j=1}^{N} \frac{p\left(a_{i}, b_{j}\right) \log _{2}\left(\frac{p\left(a_{i}, b_{j}\right)}{p\left(r_{i}\right) p\left(f_{j}\right)}\right)}{p\left(a_{i}\right) \log _{2}\left(a_{i}\right)+p\left(a_{j}\right) \log _{2}\left(b_{j}\right)}
$$

where $\boldsymbol{p}\left(\boldsymbol{a}_{\boldsymbol{i}}\right)$ and $\boldsymbol{p}\left(\boldsymbol{b}_{\boldsymbol{j}}\right)$ are the marginal probability distribution of each image. When two images are geometrically aligned, IECC value is maximized.

\subsection{Geometric Transformations}

Different transformation models are utilized for various registration applications. Recall that, image registration consists of establishing correspondence means matching of identical shapes in related image pair. This requires geometric transformation of one image into another. Change in viewpoint or relative motion between the camera and object planes introduces distortion in the features of an image e.g. a circle may appear ellipse. However, certain features of object shape remain intact even after such transformations. These features are called invariants. The fundamental characteristic of any image registration technique is the type of spatial transformations or mapping used to properly overlay two images. The most common global transformations are rigid, similarity, affine, projective.

\subsubsection{Rigid Body Transformation}

This transformation will be in the form:

$$
\check{\mathbf{x}}=\mathbf{R x}+\mathbf{T r}
$$


where $\quad \mathbf{R}=\left[\begin{array}{rr}\cos (\theta) & -\sin (\theta) \\ \sin (\theta) & \cos (\theta)\end{array}\right]$ is the rotation matrix, and $\operatorname{Tr}=\left[\mathbf{t}_{x}, \mathbf{t}_{y}\right]^{t} \quad$ is translation vector. $\breve{\mathbf{x}}$ and $\mathbf{x}$ are the transformed and original 2D points, respectively. The Rigid transformation is invariant to lengths and angles.

\subsubsection{Similarity Transformation}

This transformation will be in the form:

$$
\check{\mathbf{x}}=\mathbf{s R \mathbf { x }}+\mathbf{T r}
$$

Here s is a scaling factor. Similarity invariants are angles, ratios of lengths, and ratios of areas.

\subsubsection{Affine Transformation}

The most commonly used registration transformation is the affine transformation which is sufficient to match two images of a scene taken from the same viewing angle but from different position. It is composed of scaling, translation, and rotation. It is global transformation which is rigid. Affine transformations are more general than rigid. The general 2D affine transformation:

$$
\check{\mathbf{x}}=\mathbf{S R x}+\mathbf{T r}
$$

where $\mathbf{S}=\left[\begin{array}{ll}\boldsymbol{s}_{\boldsymbol{x}} & \mathbf{0} \\ \mathbf{0} & \boldsymbol{s}_{\boldsymbol{y}}\end{array}\right]$ is the scaling matrix. Angles and lengths are not preserved. Parallel lines remain parallel.

\subsubsection{Projective Transformation}

This is the most general geometric transformation. Here, two 2D points $\breve{\mathbf{x}}$ and $\mathbf{x}$ (represented in homogeneous coordinates), are related by a $3 \times 3$ non-singular transformation matrix: 


$$
\left[\begin{array}{l}
\check{\mathbf{x}} \\
\mathbf{1}
\end{array}\right]=\mathbf{A}\left[\begin{array}{l}
\mathbf{x} \\
\mathbf{1}
\end{array}\right]
$$

where

$$
\mathrm{A}=\left[\begin{array}{lll}
h_{11} & h_{12} & h_{13} \\
h_{21} & h_{22} & h_{23} \\
h_{31} & h_{32} & 1
\end{array}\right]
$$

Projective invariants include the cross ratio of four collinear points, or four concurrent lines [44].

\subsection{Optimization}

Given a similarity measure, $\mathcal{D}\left(I_{1}, I_{2}\right)$, and a family of geometric transformations, $W$, registration is merely an optimization problem:

$$
A^{*}=a \operatorname{rgma} \underset{A}{ } \mathcal{D}\left(I_{1}, I_{2} \circ \mathrm{A}\right)
$$

Some methods, e.g. Fourier based algorithms [45] that deal with simple transformation spaces (e.g. translation only) and simple alignment measures (e.g. SSD), can solve (4.21) directly. Most methods, on the other hand, do not enjoy a well-behaved, low dimensional objective function. Typically, registration algorithms attempt to solve the optimization using an iterative strategy. For a detailed survey, see [38]. With the family of global transformations, the goal is to search for the optimum set of transformation parameters values. Note that the similarity measure gradient (with respect to transformation parameters) is commonly used to speed up this search. Popular choices of optimizers are: gradient-descent and its variants [34], Powell's method [37], Downhill simplex method and Levenberg Marquardt optimization [38]. 


\subsection{Experimental Results}

This section compares the accuracy and the execution time of the different similarity measures. A test set of 200 images is used with $512 \times 512$ pixels in the experiment (Google search). All images are converted to gray scale. All these images represent the reference images. The other set of images for registration (the floating images) is constructed by applying various degradations to the images from the test set. In this way, various changes in the image acquisition process are simulated (such as a change in the position of the light source or a change of the sensor type), assuming the same scene has been imaged [35]. By utilizing the same registration process and changing only the image similarity measure, the registration accuracy will reflect the accuracy of the image similarity measure. This experiment was independently repeated for image registration using translation and scaling transformation. We compare between seven different measures: SSD, NCC, EC, H, MI, NMI and IECC (See Section 4.2).

For the translation, the similarity measure is calculated for a shift of \pm 100 pixels, with a step of one pixel. For the scaling, the scaling factor is calculated for the interval $[0.5,2]$, with a step size of 0.1 . In all experiments the similarity measure is calculated in the overlapping image region only, i.e. the set $D_{\mathrm{x}}$ is defined as $\boldsymbol{F} \cap \boldsymbol{R}$, and both translation and scaling are done in the y-axis direction only. The exhaustive search for the maximum, instead of implementing an optimization algorithm, is done to be sure that the global maximum and the correct alignment is achieved.

The average registration error and standard deviation for the translation registration (in pixels) are show in Table 4.1. We can notice that the first row (average) reflects the 
accuracy of the similarity measure, showing a larger bias for some similarity measures (e.g. $N C C$ ). The second row (standard deviation) reflects the image similarity measure precision, showing that IECC measure is much accurate and precise than others.

For the scaling, the accurate registration is achieved for a scaling factor of one. In this case the registration error is measured as [35]:

$$
\text { Error }=\log _{2}(1+\epsilon)
$$

where $\boldsymbol{\epsilon}$ is the absolute error from the accurate registration result. The logarithm is introduced to assure that the scaling error is symmetrical, i.e. it gives the same error for shrinking and stretching the image by the same factor. Also it gives no error if the images are scaled by the same factor. The average registration error and standard deviation for the scaling registration are show in Table 4.2.

To evaluate the execution time of the similarity measures, the Matlab ${ }^{\circledR} 7$ was used ${ }^{1}$. The average execution time from 200 function calls is used to compare the performance of the similarity measures. The results of the experiment are shown in Table 4.3, which presents the average execution time of the different image similarity measures. From the data we can notice that $E C$ is faster than $H, N M I$ and $I E C C$, and almost as fast as $N C C$, but slower than $S S D$.

Table 4.1. Average and SD of the translation registration error for various types of image similarity measures.

\begin{tabular}{|c|c|c|c|c|c|c|c|}
\hline & $S S D$ & $N C C$ & $E C$ & $H$ & $M I$ & NMI & IECC \\
\hline $\begin{array}{c}\text { Average } \\
\text { Error } \\
\text { (in pixel) }\end{array}$ & 7.22873 & 12.37281 & 2.98327 & -3.81182 & -1.16434 & -0.99451 & -0.74560 \\
\hline $\begin{array}{c}\text { Standard } \\
\text { Deviation }\end{array}$ & 87.87301 & 68.37853 & 41.73810 & 42.67132 & 28.95493 & 27.37621 & 22.06574 \\
\hline
\end{tabular}

${ }^{1}$ All algorithms are run on a PC with a $2 \mathrm{GHz}$ Core i7 Quad processor with 6GB RAM. 
Table 4.2. Average and SD of the scaling registration error for various types of image similarity measures.

\begin{tabular}{|c|c|c|c|c|c|c|c|}
\hline & $S S D$ & $N C C$ & $E C$ & $H$ & $M I$ & $N M I$ & $I E C C$ \\
\hline $\begin{array}{c}\text { Average } \\
\text { Error }\end{array}$ & 0.40675 & 0.20147 & 0.13237 & 0.13242 & 0.10876 & 0.0999 & 0.0422 \\
\hline $\begin{array}{c}\text { Standard } \\
\text { Deviation }\end{array}$ & 0.7327 & 0.7285 & 0.60362 & 0.42384 & 0.45317 & 0.43389 & 0.34563 \\
\hline
\end{tabular}

Table 4.3. Average excusion time (in miliseconds) for each similarity measure.

\begin{tabular}{|c|c|c|c|c|c|c|c|}
\hline & $S S D$ & NCC & $E C$ & $H$ & $M I$ & NMI & IECC \\
\hline $\begin{array}{c}\text { Time } \\
\text { (in msec) }\end{array}$ & 4.301 & 14.287 & 15.987 & 43.733 & 44.189 & 43.572 & 45.613 \\
\hline
\end{tabular}

These results indicate that: For the interventional applications, although $E C$ framework is not the most accurate, it has the advantage of reducing the execution time by almost two third as compared to $N M I$ and IECC. On the other hand, if the accuracy is sought, IECC outperforms the other systems with comparative execution time to NMI.

\subsection{Summary}

Image registration is one of the most important tasks when integrating and analyzing information from various sources. It is a key stage in image fusion, change detection, super-resolution imaging, and in building image information systems, among others. This chapter gives a survey of the classical and up-to-date registration methods, classifying them according to their nature as well as according to the three major registration steps. Although a lot of work has been done in this area, automatic image registration still remains an open problem. Registration of images with complex nonlinear and local distortions, multimodal registration, and registration of N-D images (where $\mathrm{N}>$ 2) belong to the most challenging tasks at this moment. 


\section{PART II}

\section{APPLICATIONS FOR MEDICAL IMAGE ANALYSIS}




\section{CHAPTER 5}

\section{SHAPE BASED SEGMENTATION OF THE VERTEBRAL BODY}

Isolating an organ from its surrounding anatomical structures is a crucial step in many unsupervised frameworks. Examples of these frameworks are those that assess the organ functions and those that are proposed for automatic classification of normal organ and acute rejection transplants. In this work, we propose segmentation frameworks for spine bone, more specifically the Vertebral Body (VB).

Segmentation can be defined as partitioning the image into the meaningful areas using the existing (low level) information in the image and prior (high level) information which can be obtained using a number of features of an object. The human vision system aims to extract and use as much as possible information in the image. This information includes the intensity, motion of the object (in sequential images), spatial relations (interaction) as the existing information, and the shape of the object which is learnt from the experience as the prior information. The machine visual system cannot predict the prior information unless it is supplemented. Hence, any prior cue can be specified beforehand to enhance the segmentation or to obtain the desired segmentation. If the prior information of the object is not given beforehand to the machine vision task, the segmentation method may not give desired results due to noise, occlusion, and missing information in the image.

One of the bone diseases, which is characterized by a reduction in bone mass, is osteoporosis. This disease results in an increased risk of fractures. Bone Mineral Density 


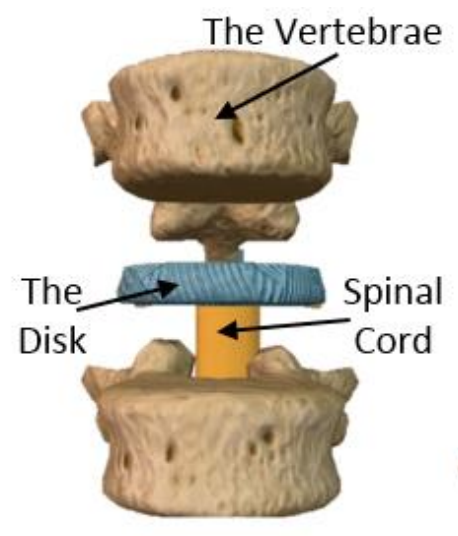

(a)

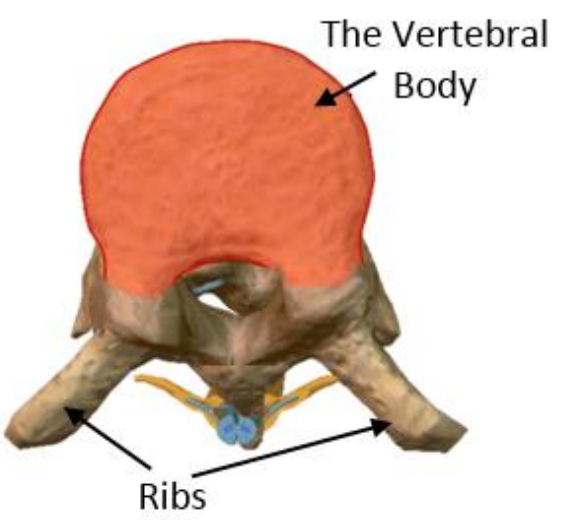

(b)

Figure 5.1. The region of interest in the experiment, (a) The anatomical structure of the spine, (b) The red color shows the VB region. Bone Mineral Density (BMD) measurements and Fracture Analysis (FA) are restricted to the VB.

(BMD) measurements and Fracture Analysis (FA) of the VBs should be obtained to accurately diagnose the osteoporosis. To obtain these measurements and analysis, VBs should be correctly segmented, which is the main objective in this chapter.

Since BMD measurements and fracture analysis are restricted to the vertebral bodies, segmentation approaches should successfully isolate VB from processes, which constitute spine bone as shown in Figure 5.1. However, due to region inhomogeneities existing in CT images, isolating a VB from its background is not an easy task as shown in Figure 5.2. To overcome these inhomogeneities and accurately segment VBs, we use both shape and appearance information.

The literature is rich with organ segmentation techniques. However, in this chapter, we will review and introduce only some of these techniques whose basics depend on shape modeling and whose application is VB segmentation. 


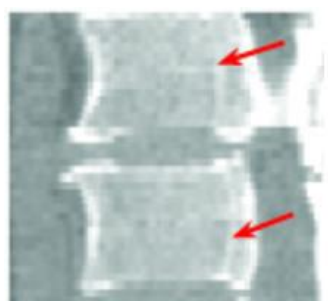

(a)

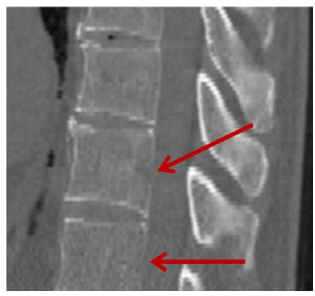

(e)

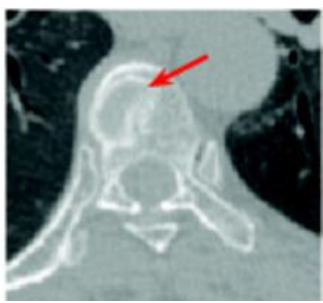

(b)

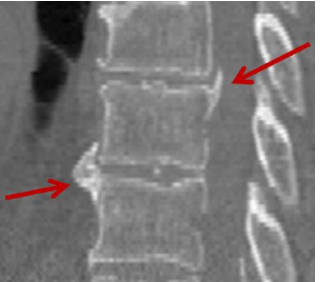

(f)

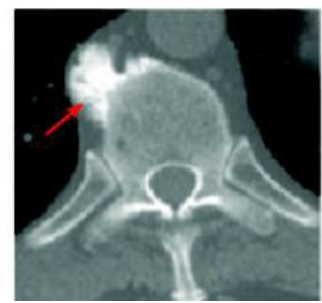

(c)

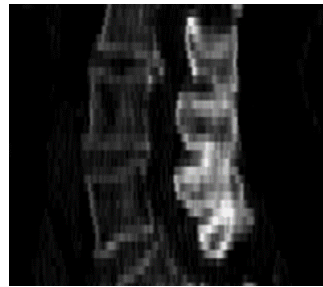

(g)

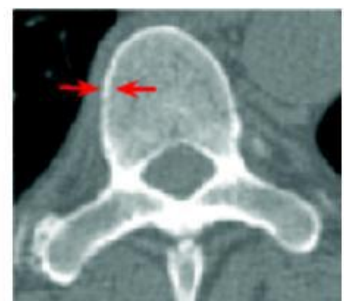

(d)

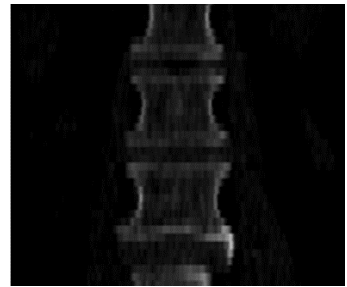

(h)

Figure 5.2. Typical challenges for vertebrae segmentation: (a) Inner boundaries. (b) Bone degenerative disease. (c) Osteophytes. (d) Double boundary, (e) weak bone edges, (f) osteophytes, and $(\mathrm{g}-\mathrm{h})$ low resolution.

\subsection{Anatomy of the Spine Column and Osteoporosis}

There are five sections of the spinal column including the cervical (7 VBs), thoracic (12 VBs), lumbar (5 VBs), sacral (5 VBs), and coccyx (3-5 fused VBs) as shown in Figure 5.3. The VB consists of cortical and trabecular regions. Cortical and trabecular bones form $70-80 \%$ and $20-30 \%$ of bone mass, respectively. Approximately $25 \%$ of the trabecular bone volume is bone tissue and $75 \%$ is bone marrow and fat. This proportion changes between different parts of the skeleton. Bone marrow has stroma, myeloid tissue, fat cells, blood vessels, sinusoids and some lymphatic tissue. The ratio between bone tissue and bone marrow also decreases with osteoporosis [103].

Osteoporosis is a bone disease characterized by a reduction in bone mass, resulting in an increased risk of fractures [103]. Figures 5.4 and 5.5 shows some example views of 


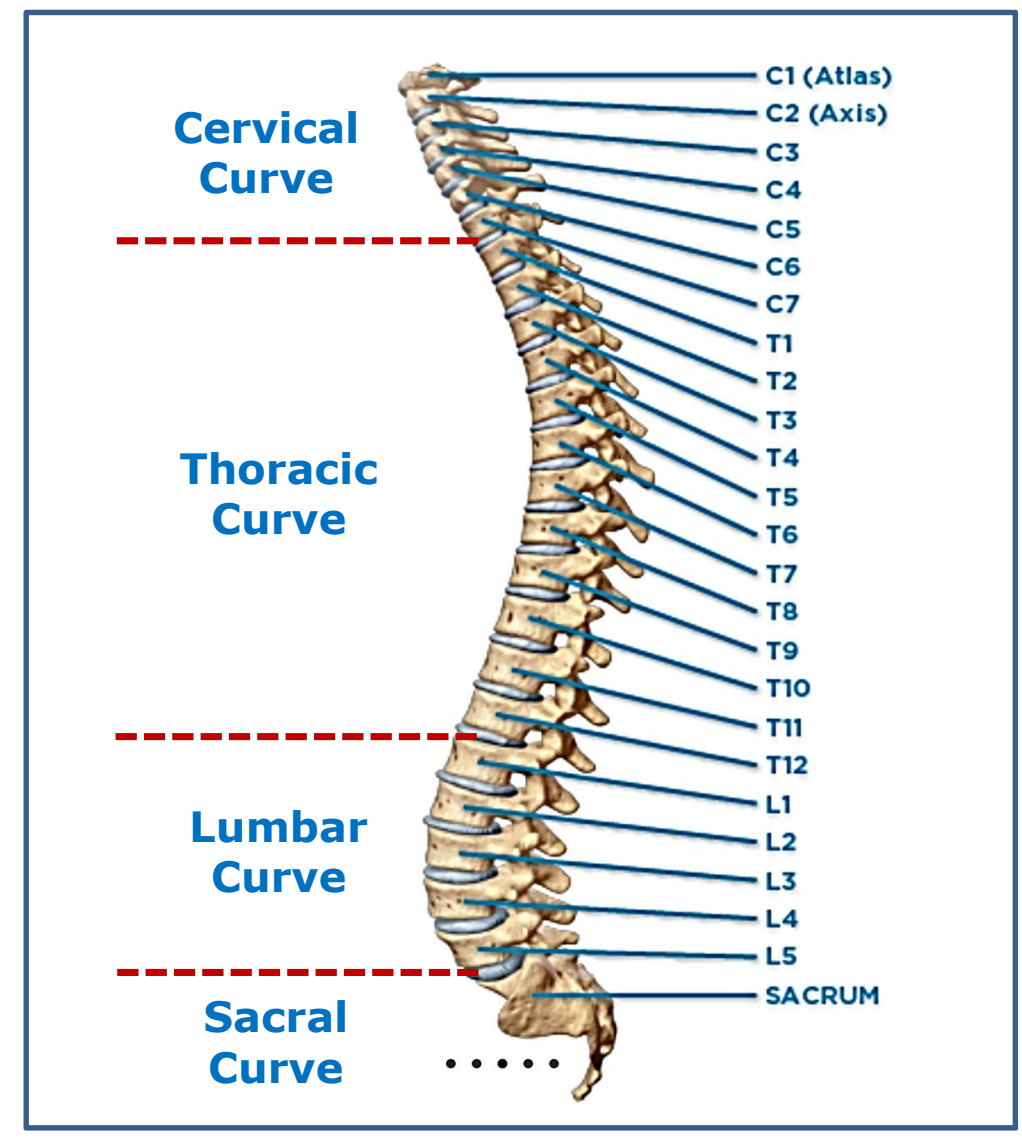

Figure 5.3. The sagittal view of the spine column. There are five regions of the spine column: cervical, thoracic, lumbar, and sacrum.

healthy, osteopenia, and osteoporosis bones. With osteoporosis, a subject's bone tissue has less than the normal proportion amount of calcium. The additional space is filled with fat. The ratio between the bone tissue and bone marrow is decreasing [103]. Low bone mass and osteoporosis occur more frequently in women. The bone begins loosing its weight and calcium soon after menopause. Without diagnosis and prevention, a woman can lose $20 \%$ $30 \%$ of her bone mass during the first 10 years of menopause [104]. 


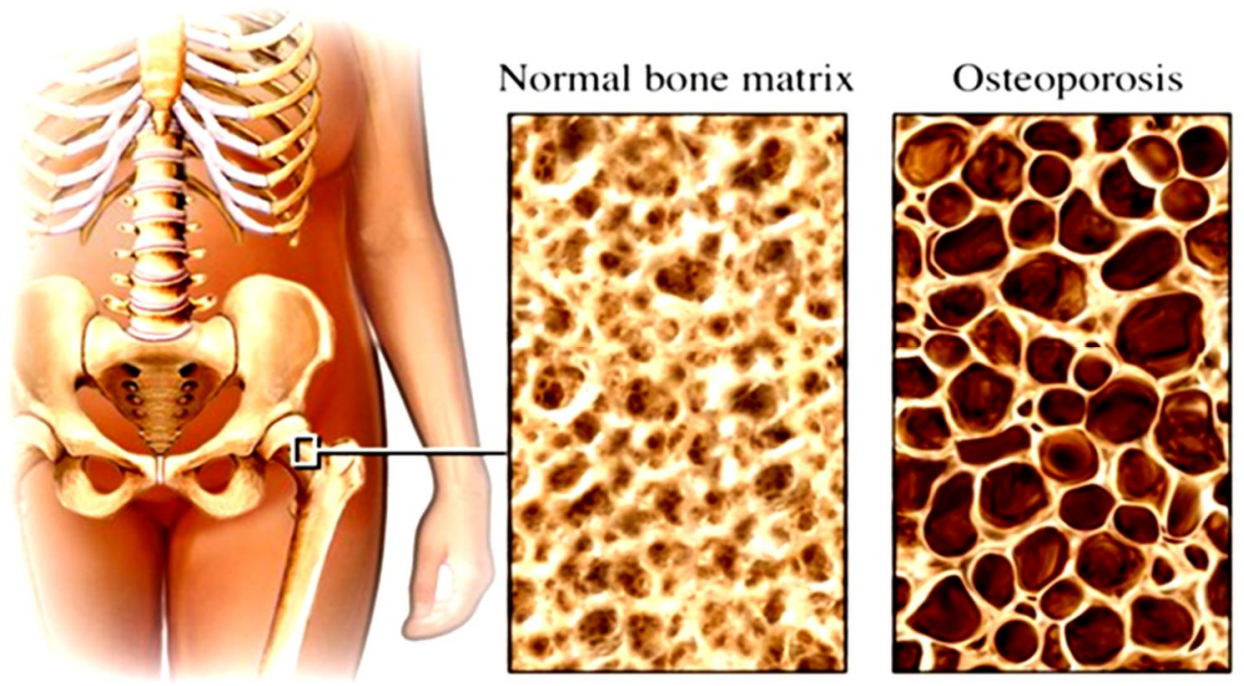

Figure 5.4. Differences of the healthy and osteoporosis bone. (This image is adopted from [108]).

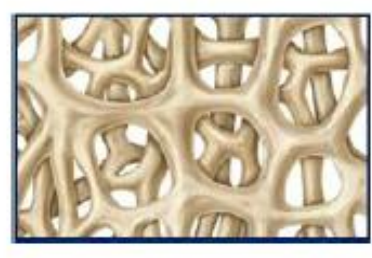

(a)

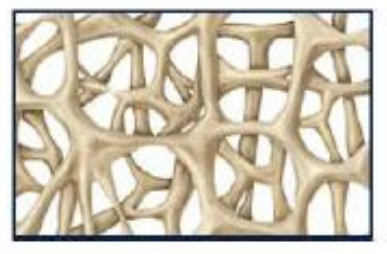

(b)

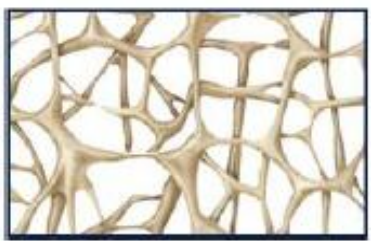

(c)

Figure 5.5. Three different bone tissues. (a) Healthy, (b) osteopenia, and (c) osteoporosis bones. (These images are adopted from [104]).

Based on the Surgeon General report [104], there were approximately 10 million people over age 50 with osteoporosis and an additional 34 million with low bone mass or osteopenia in the United States in 2002. Unfortunately, the total number is expected to be increased to 61.4 million in 2020 as in [104]. These changes could cause the number of vertebrae, hip, and wrist fractures to increase rapidly by 2040 . It should be noted that $50 \%$ from all osteoporotic fractures are vertebral [105]. 
Doctors need the BMD measurements of vertebral bones in order to diagnose and treat osteoporosis. The BMD measurements remain the 'gold standard' test for an osteoporosis diagnosis. The BMD measurements are strong predictors of fracture risk. In the Surgeon General's report, it is strongly stated that the relationship between the BMD score and future fracture is stronger than the relationship between cholesterol and heart attack [60]. The BMD measurements are also used to assess bone changes in treated and untreated individuals for monitoring drug therapies.

The rest of this chapter is organized as follows: Section 5.2 discusses the background of methods and related work to our problem. Section 5.3 and 5.4 introduces the general framework of proposed approaches and the pre-processing step required for the framework, respectively. Section 5.5 and 5.6 explain in details the proposed 2D and 3D approaches for VB segmentation, respectively, and compares the results with other alternatives. Applications on bone mineral density (BMD) measurements of vertebral body are given at Section 5.7. Finally, summary is drawn in Section 5.8

\subsection{A review on VB Segmentation Methods and Related Work}

As it has been discussed before, the vertebra consists of the VB, spinous (spinal) processes, pedicles, and other anatomical regions (see Figure 5.1). Spinous processes, pedicles, and ribs should not be included in the BMD measurements since the BMD measurements are restricted to the VBs. As shown in figures, the VB segmentation is not an easy task since the ribs and spinal processes have similar gray level information.

The literature is rich with organ segmentation techniques. However, we will discuss only some of these techniques whose basics depend on shape modeling and whose 
application is VB segmentation. To tackle the problem of segmenting spine bones, various approaches have been introduced. For instance, Klinder et al. [107] developed an automated model-based vertebra detection, identification and segmentation approach. Kang et al. [53] developed a 3D segmentation method for skeletal structures from CT images. Their method starts with a three dimensional region growing step using local adaptive thresholds. Then a closing of boundary discontinuities and an anatomicallyoriented boundary adjustment steps are done. They presented various anatomical bony structures as applications. They evaluated their segmentation accuracy using the European Spine Phantom (ESP) [54]. In order to measure bone mineral density, Mastmeyer et al. [55] presented a hierarchical segmentation approach for the lumbar spine. They reported that it takes less than $10 \mathrm{~min}$ to analyze three vertebrae, which is a huge improvement compared to what is reported in [56]: 1-2 hours. However, this framework needs excessive user interaction to precisely locate seed points to facilitate region growing segmentation. This process is time consuming and impractical for unhealthy bone segmentation. To analyze the fracture of VBs, Roberts et al. [106] used the active appearance model. Other techniques have been developed to segment skeletal structures and can be found for instance in [108-110].

Actually, there are a huge number of segmentation techniques in the literature: simple techniques (e.g. region growing or thresholding), parametric deformable models and geometrical deformable models. However, all these methods tend to fail in the case of noise, gray level inhomogeneities, and diffused boundaries. Organs have well-constrained forms within a family of shapes. Therefore segmentation algorithms have to exploit the 
prior knowledge of shapes and other properties of the structures to be segmented. Leventon et al. [111] combined the shape and deformable model by attracting the level set function to the likely shapes from a training set specified by principal component analysis (PCA). To make the shape guides the segmentation process, Chen et al. [112] defined an energy functional, which basically minimizes a Euclidean distance between a given point and its shape prior. Huang et al. [1], combined registration with segmentation in an energy minimization problem. The evolving curve is registered iteratively with a shape model using the level sets. They minimized a certain function to estimate the transformation parameters.

For 2D segmentation of the VB in CT images, the group in CVIP lab proposed various methods to segment VBs in [123]-[62] which can be considered as progressive VB segmentation studies. In [123], the shape model was not used and it was assumed that the detection rate of VBs was very accurate for cropping the pedicles automatically. In [52], a probabilistic shape model was introduced in addition to the intensity and spatial interaction information to enhance the results. However, the shape model was assumed to be registered to the object of interest manually. In [58]-[62], the probabilistic shape model was automatically embedded into image domain and they appeared to be more realistic experiments. In [61], the Chen-Vese level sets method which needs manual initialization was used, and was validated on a limited number of data sets. In [58], the shape prior is extracted using PCA on signed distance functions (SDF) of all training images. Then the shape model was registered into the image domain using the gradient descent approach [59]. 


\subsection{The Proposed Framework}

Intensity models may not be enough to obtain the optimum segmentation. Hence, a shape based iterative segmentation method is proposed. Figure 5.6 summarizes the main components of the typical framework. This framework contains three phases. In the first phase, a statistical shape model of human VB is obtained. Shape information is gathered from a set of training shapes.

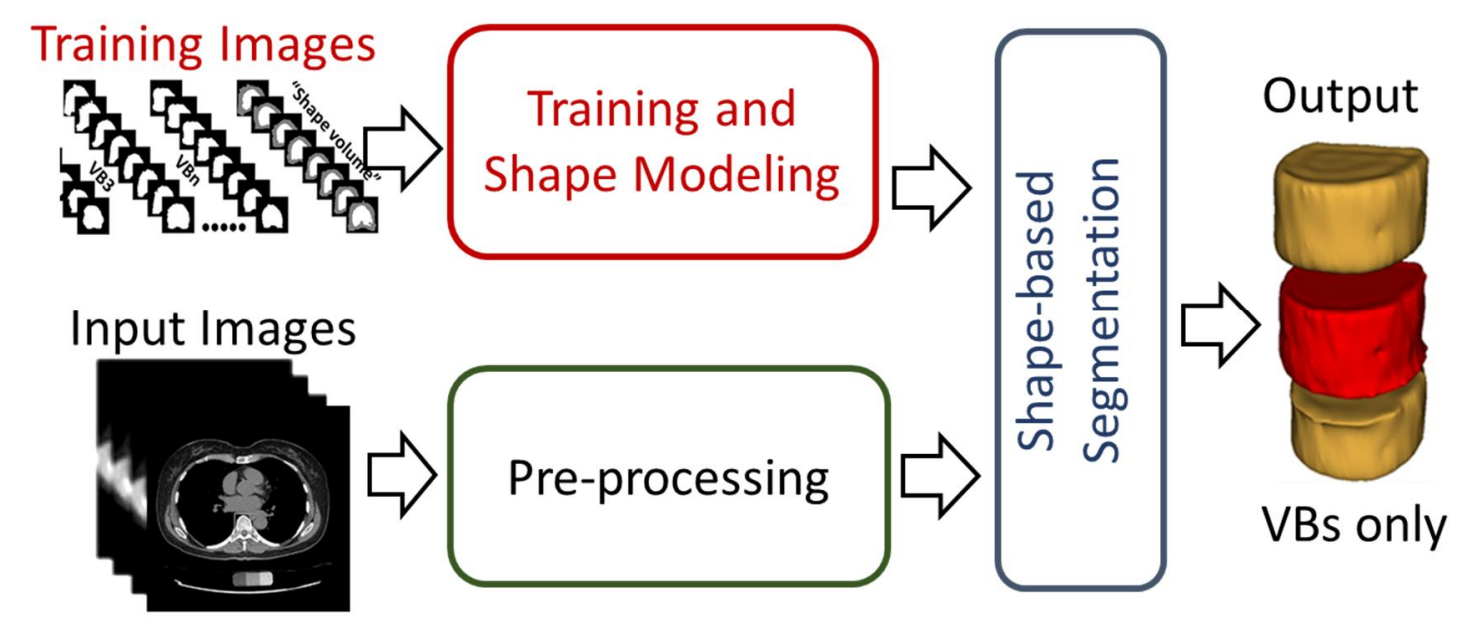

Figure 5.6. A General block diagram of the proposed framework.

To eliminate the user interaction and to improve the segmentation accuracy and minimize the execution time, the human spine area is extracted using the Matched filter (MF) and each vertebrae of the input CT images is automatically separated. This second phase is considered as a pre-processing step.

In the third phase, a curve/disk as the initial evolving front on the VB image/volume is initialized. Then, an iterative process begins to segment the desired VB. This phase can be considered as the main core of the proposed framework. 
In this chapter, two different segmentation approaches are introduced. Both of them are obeying the variational shape-based segmentation frameworks described in Figure 5.6.

The first approaches deals with two dimensional (2D) case. This segmentation approach starts with obtaining the initial segmentation using the intensity/spatial interaction models. Then, shape model is registered to the image domain. Finally, the optimal segmentation is obtained using the optimization of an energy functional which integrating the shape model. The shape variations are modelled using two-dimensional principal component analysis (2D-PCA). The proposed method is tested on the synthetic and clinical images/shapes and it is shown to be robust under various noise levels and missing object information. The proposed shape based segmentation methods are less variant to the initialization.

The second approach is a three dimensional (3D) simultaneous segmentation and registration approach. The information of the intensity are handled by embedding an edgemounted Willmore flow into the level set segmentation framework. Then the shape variations are estimated using a new distance probabilistic model which approximates the marginal densities of the vertebral body and its background in the variability region using a Poisson distribution. The experimental results show that the segmentation accuracy of this framework are much higher than other alternatives. This study reveals that the proposed method is robust under various noise levels and completely eliminates the user interaction. Applications on bone mineral density (BMD) measurements of vertebral body are given to illustrate the accuracy of the proposed segmentation approach. More details about these two system will be discussed in Section 5.5 and 5.6, respectively. 


\subsection{The Pre-processing Phase and Detection of ROI}

To roughly remove the spinal processes and ribs and to detect the region of interest (ROI), i.e. VB region, some simple procedures are adopted as a pre-processing step. This step is required to: i) remove the unwanted anatomical structures simplifying the segmentation process, ii) crop the ROI minimizing the execution time, and iii) separate the vertebra in the input $\mathrm{CT}$ images eliminating the user interaction. Details on these procedures will be discussed in the following subsections.

\subsubsection{Spine Cord Extraction}

As a pre-processing step, the spinal cord is extracted using the Matched filter (MF). This process helps to roughly remove the spinous processes and pedicles. Additionally, it eliminates the user interaction and improves the segmentation accuracy. Let $\boldsymbol{f}(\boldsymbol{x}, \boldsymbol{y})$ and $\boldsymbol{g}(\boldsymbol{x}, \boldsymbol{y})$ be template and test images, respectively. To compare the two images for various possible shifts $\boldsymbol{\tau}_{\boldsymbol{x}}$ and $\boldsymbol{\tau}_{\boldsymbol{y}}$, one can compute the cross-correlation $\boldsymbol{c}\left(\boldsymbol{\tau}_{\boldsymbol{x}}, \boldsymbol{\tau}_{\boldsymbol{y}}\right)$ as

$$
c\left(\tau_{x}, \tau_{y}\right)=\iint g(x, y) f\left(x-\tau_{x}, y-\tau_{y}\right) d x d y,
$$

where the limits of integration are dependent on $\boldsymbol{g}(\boldsymbol{x}, \boldsymbol{y})$. The (5.1) can also be written as

$$
c\left(\tau_{x}, \tau_{y}\right)=F T^{-1}\left(G\left(f_{x}, f_{y}\right) F^{*}\left(f_{x}, f_{y}\right)\right),
$$

where $\boldsymbol{G}\left(\boldsymbol{f}_{\boldsymbol{x}}, \boldsymbol{f}_{\boldsymbol{y}}\right)$ and $\boldsymbol{F}\left(\boldsymbol{f}_{\boldsymbol{x}}, \boldsymbol{f}_{\boldsymbol{y}}\right)$ are the 2D Fourier Transform (2DFT) of $\boldsymbol{g}(\boldsymbol{x}, \boldsymbol{y})$ and $\boldsymbol{f}(\boldsymbol{x}, \boldsymbol{y})$, respectively with $\boldsymbol{f}_{\boldsymbol{x}}$ and $\boldsymbol{f}_{\boldsymbol{y}}$ denoting the spatial frequencies. The test image $\boldsymbol{g}(\boldsymbol{x}, \boldsymbol{y})$ is filtered by $\boldsymbol{H}\left(\boldsymbol{f}_{\boldsymbol{x}}, \boldsymbol{f}_{\boldsymbol{y}}\right)=\boldsymbol{F}^{*}\left(\boldsymbol{f}_{x}, \boldsymbol{f}_{\boldsymbol{y}}\right)$ to produce the output $\boldsymbol{c}\left(\boldsymbol{\tau}_{\boldsymbol{x}}, \boldsymbol{\tau}_{\boldsymbol{y}}\right)$.

Hence, $\boldsymbol{H}\left(\boldsymbol{f}_{\boldsymbol{x}}, \boldsymbol{f}_{\boldsymbol{y}}\right)$ is the correlation filter which is the complex conjugate of the 2DFT of the reference image $\boldsymbol{f}(\boldsymbol{x}, \boldsymbol{y})$. Figure 5.7(a) shows the reference image used in the MF. Some 


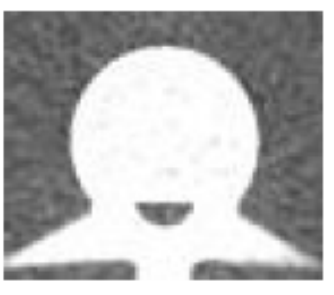

(a)

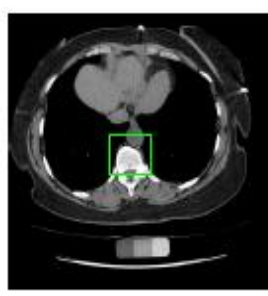

(b)

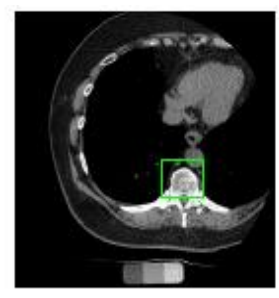

(c)

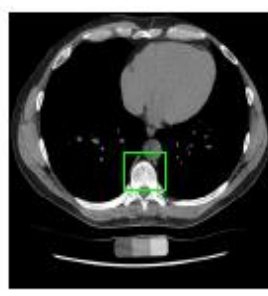

(d)

Figure 5.7. (a) The template used for the Matched filter, (b-d) a few images of automatic VB detection. The green line shows the detection of VB region.
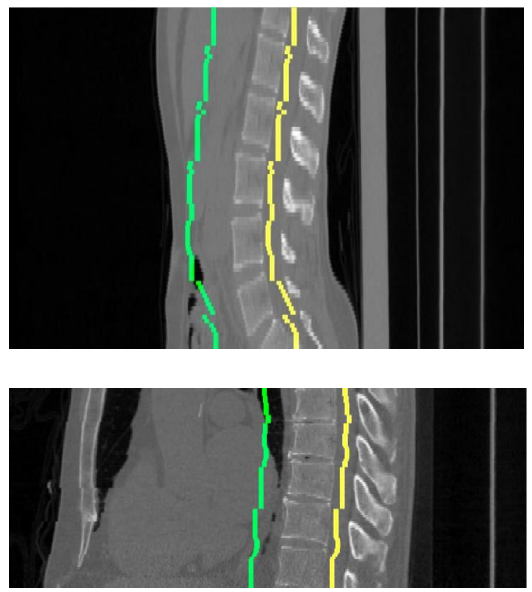

(a)
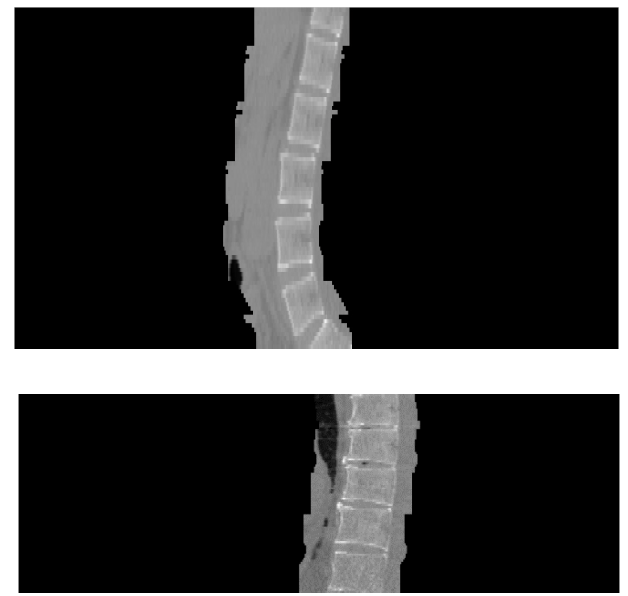

(b)
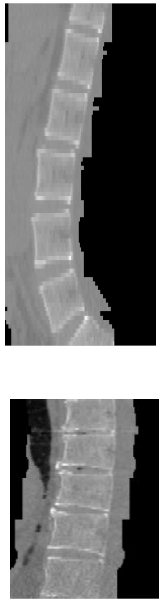

(c)

Figure 5.8. Examples for the extraction of the spinal cord on a data set (a) The detected VB region, (b) The refined data to extract the spinal processes and ribs. and (c) The cropped data to reduce the size of the image.

examples of the VB detection are shown in Figs. 5.7(b-d). The MF is tested using 3000 clinical CT. The detection accuracy for the VB region is $97.6 \%$. The detection accuracy is increased to around $100 \%$ by smoothing all detected points of a dataset in the z-axis. After detecting the VB region, the ROI is cropped to minimize the execution time. Figures 5.8 shows different examples of this stage in the sagittal view.

\subsubsection{Vertebrae Separation}

To separate the VBs, the previously developed approach based on four points automatically placed on cortical shell is used [113]. A demonstration of the separation process of a VB is shown in Figure 5.9. Because cortical bone has higher gray level 


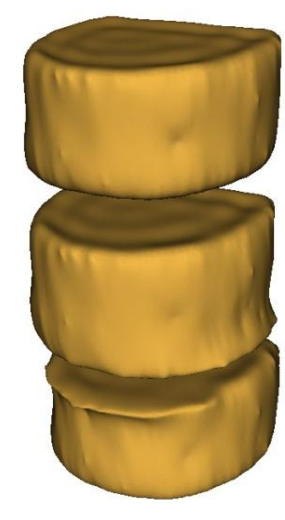

(a)

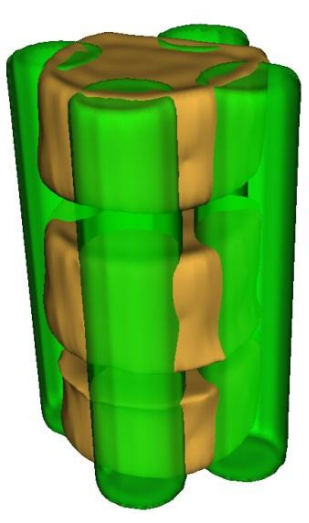

(b)

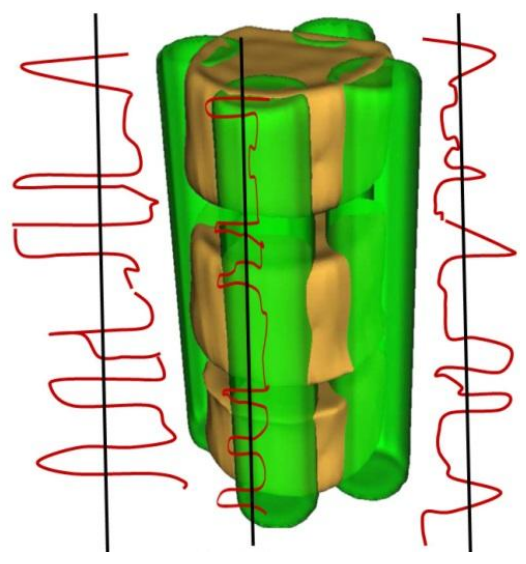

(c)

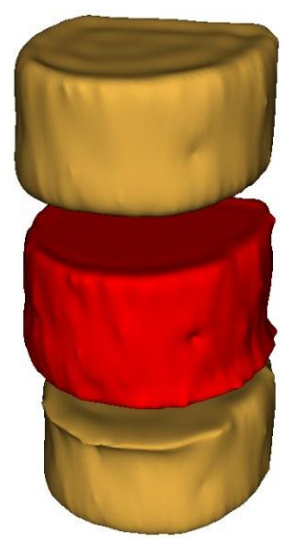

(d)

Figure5.9. The separation of the VB region. (a) 3D view of three adjacent VB, (b) automated placement of 4 seeds on cortical bone and disc,(c) The histograms (red lines) and the thresholds (black lines) calculation along each detected seed, (d) separation of VB shown with red color.

intensity than trabecular bone and surrounding tissue, the boundary of the VB can be easily obtained. After cortical bone is obtained; four seeds are automatically placed. These seeds are placed using the relatively higher gray level intensity values of the cortex region images.

Next, the histogram for a neighborhood around each seed is obtained. The histogram represents the number of voxels whose intensity values are above 200 Hounsfield Unit (HU). This value is obtained empirically. Vertical boundaries of a VB show higher gray level intensity than inner region of the VB and disks. Figure 5.9(c) shows histograms (the red line), and thresholds (the black line). To search vertical limits of the VB, the following adaptive threshold equation is used as follows:

$$
\boldsymbol{T H}=\boldsymbol{\mu}(\boldsymbol{A})+\boldsymbol{\kappa} *[\max (\boldsymbol{A})-\boldsymbol{\mu}(\boldsymbol{A})],
$$

where $\kappa=0.3$ which is derived from experiments by trial-and-error, where A represents each histogram vector with the red line, $\max (A)$ and $\mu(A)$ are the maximum and average values in the histogram vector. In this step, 40 patients which totals to $153 \mathrm{VBs}$ are used. The 
proposed method produced about $89.3 \%$ successful separation results.

\subsection{Two Dimensional Approach for VB Segmentation}

In this method and after the pre-processing phase, initial labeling $\left(f^{*}\right)$ is obtained using the graph cuts which integrates the intensity and spatial interaction models, as shown in Figure 5.10 . Finally, the initial labeled image and the shape priors are registered to obtain the optimum labeling, as in [59]. To obtain the shape priors (p), the 2D-PCA is used on all training images. Figure 5.11 summarizes the main components of this framework. The following sections give more details about the shape model construction and the segmentation method

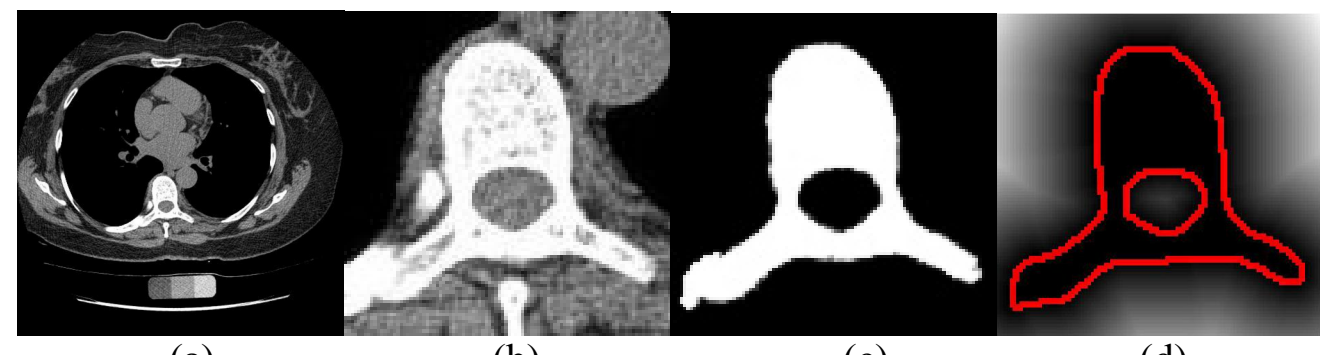

(a)

(b)

(c)

(d)

Figure 5.10. An example of the initial labeling. (a) Original CT image, (b) detection of the $\mathrm{VB}$ region using MF, (c) the initial labeling, $\mathbf{f}^{*}$ and (d) the SDF of the initial segmentation $\left(f^{*}\right)$ which is used in the registration phase. Red color shows the zero level contour.

\subsubsection{Shape model construction}

In this work, the shape representation using the SDF is described. The objective of this step is to obtain the most important information of training images using 2D-PCA. As opposed to conventional PCA, 2D-PCA is based on 2D matrix rather than $1 \mathrm{D}$ vector. This means that, the image does not need to be pre-transformed into a vector [65]. 
In addition, the image covariance matrix $(\mathrm{G})$ can be directly constructed using the original image matrices. As a result, 2D-PCA has two important advantages over PCA. First, it is easier to evaluate $\mathrm{G}$ accurately since its size using 2D-PCA is much smaller. Second, less time is required to determine the corresponding eigenvectors [51].

2D-PCA projects an image matrix $\mathrm{X}$, which is an $m \times n$ matrix onto a vector, $\mathrm{b}$, which is an $n \times 1$ vector, by the linear transformation.

$$
\mathbf{y}=\mathbf{X b} \text {. }
$$

Suppose that there are $M$ training images, the $i^{\text {th }}$ training image is denoted by $\mathbf{X}_{\boldsymbol{i}},(i=1,2, \ldots, M)$ and the average image of all training samples is denoted by $\overline{\mathbf{X}}=\frac{\mathbf{1}}{\boldsymbol{M}} \sum_{\boldsymbol{i}=\mathbf{1}}^{\boldsymbol{M}} \mathbf{X}_{\boldsymbol{i}}$. Then, let us define the image covariance matrix G [51]:

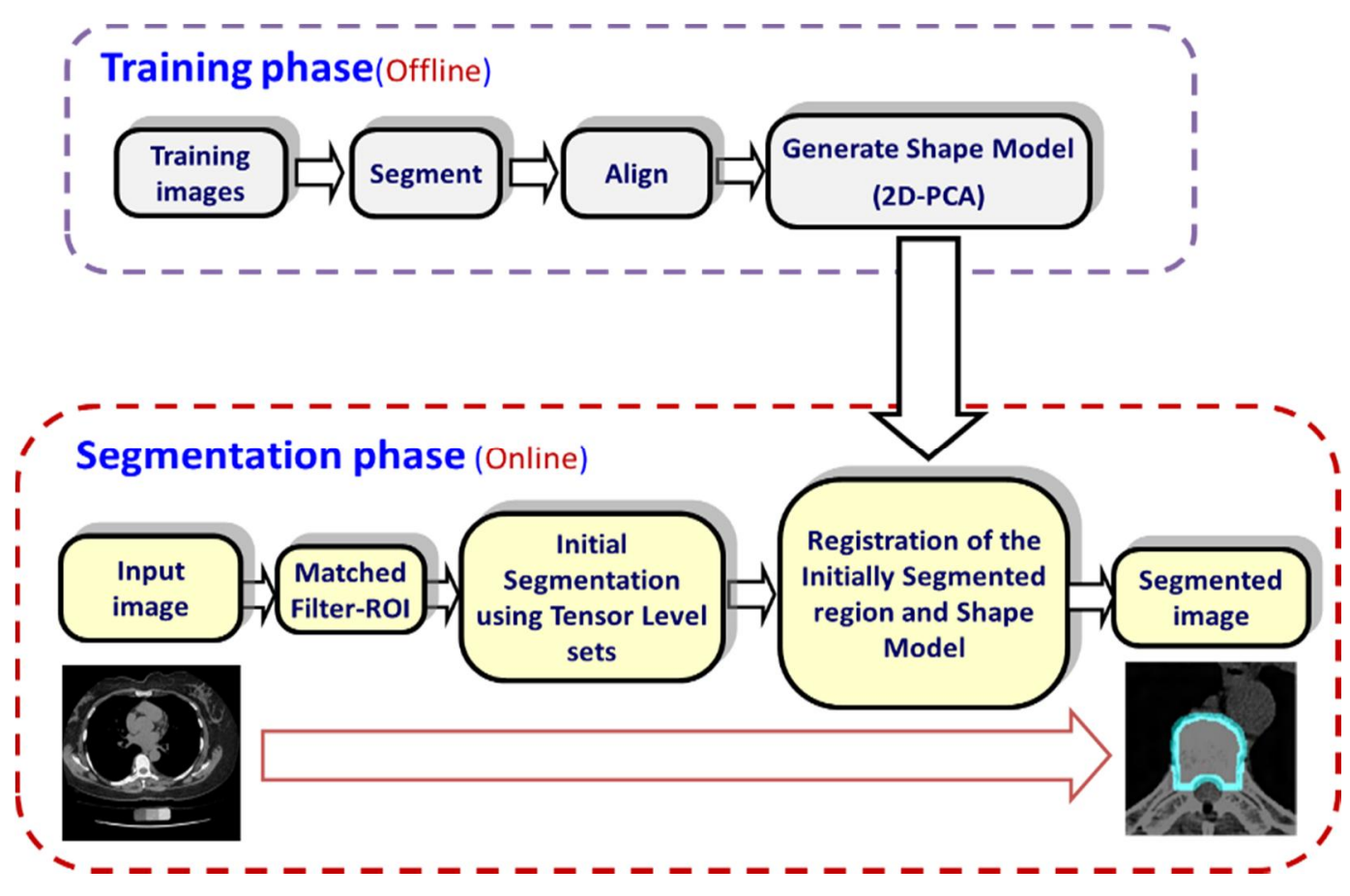

Figure 5.11. The proposed 2D shape-based segmentation framework. 


$$
\mathbf{G}=\frac{1}{M} \sum_{i=1}^{M}\left(\mathbf{X}_{i}-\overline{\mathbf{X}}\right)^{t}\left(\mathrm{X}_{i}-\overline{\mathbf{X}}\right)
$$

It is clear that, the matrix $\mathrm{G}$ is $n \times n$ nonnegative definite matrix.

Similar to PCA, the goal of 2D-PCA is to find a projection axis that maximizes $\mathbf{b}^{t} \mathbf{G b}$. The optimal $K$ projection axes $\mathrm{b}_{k}$, where $k=1,2, \ldots, K$, that maximize the above criterion are the eigenvectors of $\mathrm{G}$ corresponding to the largest $K$ eigenvalues. For an image $\mathrm{X}$, its reconstruction $\widetilde{\mathbf{X}}$ defined below is used to approximate it.

$$
\widetilde{\mathbf{X}}=\overline{\mathbf{X}}+\sum_{k=1}^{K} \mathbf{y}_{k} \mathbf{b}_{k}{ }^{t},
$$

Where $\mathbf{y}_{\boldsymbol{k}}=(\mathbf{X}-\overline{\mathbf{X}}) \mathbf{b}_{\boldsymbol{k}}$ is called the $k^{\text {th }}$ principal component vector of the sample image $\mathrm{X}$. The principal component vectors obtained are used to form an $m \times K$ matrix $\mathrm{Y}=$ $\left[\mathrm{y}_{1}, \mathrm{y}_{2}, \ldots, \mathrm{y}_{K}\right]$ and let $\mathrm{B}=\left[\mathrm{b}_{1}, \mathrm{~b}_{2}, \ldots, \mathrm{b}_{K}\right]$, then (5.6) can be rewritten as:

$$
\widetilde{\mathbf{X}}=\overline{\mathbf{X}}+\mathbf{Y B}^{t} .
$$

However, one disadvantage of 2D-PCA (compared to PCA) is that more coefficients are needed to represent an image. From (5.7), it is clear that dimension of the 2D-PCA principal component matrix $\mathrm{Y}(m \times K)$ is always much higher than PCA. To reduce the dimension of matrix Y, the conventional PCA is used for further dimensional reduction after 2D-PCA.

Now, let the training set consists of $M$ training images $\left\{\mathrm{I}_{1}, \ldots, \mathrm{I}_{M}\right\}$; with SDFs $\left\{\boldsymbol{\Phi}_{1}, \ldots, \boldsymbol{\Phi}_{M}\right\}$. All images are binary, pre-aligned, and normalized to the same resolution. As in [65], the mean level set function of the training shapes, $\overline{\mathbf{\Phi}}$, is obtained as the average of these $M$ signed distance functions. To extract the shape variabilities, $\overline{\mathbf{\Phi}}$ is subtracted from each of the training SDFs. The obtained mean-offset functions can be represented as 
$\left\{\widehat{\boldsymbol{\Phi}}_{1}, \ldots, \widehat{\boldsymbol{\Phi}}_{\boldsymbol{M}}\right\}$. These new functions are used to measure the variabilities of the training images. A set of 80 training VB images with $120 \times 120$ pixels is used in the experiment. According to (5.2), the constructed matrix G will be:

$$
\mathrm{G}=\frac{1}{M} \sum_{i=1}^{M=8} \widehat{\Phi}_{i}^{t} \widehat{\Phi}_{i}
$$

The goal of 2D-PCA is to find the optimal $K$ eigenvectors of $\mathrm{G}$ corresponding to the largest $K$ eigenvalues. The value of " $K$ " helps to capture the necessary shape variation with minimum information. Experimentally, we find that, the minimum suitable value is $K=10$ [63]. Less than this value, the accuracy of the segmentation algorithm falls drastically below other alternatives. After choosing the eigenvectors corresponding to 10 largest eigenvalues, $\mathrm{b}_{1}, \mathrm{~b}_{2}, \ldots, \mathrm{b}_{10}$, the principal component matrix $\mathrm{Y}_{i}(m=120 \times K=10)$ was obtained for each SDF of the training set $(i=1,2, \ldots, 80)$. For more dimensional reduction, the conventional PCA is applied on the principal components $\left\{\overrightarrow{\mathbf{Y}}_{\mathbf{1}}, \ldots, \overrightarrow{\mathbf{Y}}_{\boldsymbol{M}}\right\}$. It should be noted that, $\overrightarrow{\mathbf{Y}}$ is the vector representation of $\mathrm{Y}$. The reconstructed components (after retransforming to matrix representation) will be:

$$
\widetilde{\mathbf{Y}}_{\{l, h\}}=\mathbf{U} \mathbf{e}_{\{l, h\}},
$$

Where $U$ is the matrix which contains $L$ eigenvectors corresponding to $L$ largest eigenvalues $\lambda_{l},(l=1,2, \ldots, L)$, and $\mathbf{e}_{\{l, \boldsymbol{h}\}}$ is the set of model parameters which can be described as[63]:

$$
\mathbf{e}_{\{l, h\}}=h \sqrt{\lambda_{l}}
$$

where $l=\{1, \ldots, L\}, h=\{-\mu, \ldots, \mu\}$, and $\mu$ is a constant which can be chosen arbitrarily (in the experiments, we chose $L=4, \mu=3$ ). The new principal components of training SDFs are 
represented as $\left\{\widetilde{\mathbf{Y}}_{\mathbf{1}}, \ldots, \widetilde{\mathbf{Y}}_{\boldsymbol{N}}\right\}$ instead of $\left\{\mathbf{Y}_{\mathbf{1}}, \ldots, \mathbf{Y}_{\boldsymbol{M}}\right\}$ where $N$ is the multiplication of $L$ and standard deviation in eigenvalues (the number of elements in $h$ ), i.e. $N=L(2 \mu+1)$ [65]. Given the set $\left\{\widetilde{\mathbf{Y}}_{1}, \ldots, \widetilde{\mathbf{Y}}_{\boldsymbol{N}}\right\}$, the new projected training SDFs are obtained as follows:

$$
\widetilde{\boldsymbol{\Phi}}_{\boldsymbol{n}}=\overline{\boldsymbol{\Phi}}+\widetilde{\mathbf{Y}}_{\boldsymbol{n}} \mathbf{B}^{\boldsymbol{t}}, \quad n=1,2, \ldots, N
$$

Finally, the shape model is required to capture the variations in the training set. This model is considered to be a weighted sum of the projected SDFs (5.8) as follows:

$$
\Phi_{\mathrm{p}}=\sum_{n=1}^{N} w_{n} \widetilde{\Phi}_{n}
$$

Let $\mathbf{w}=\left[\boldsymbol{w}_{1}, \ldots, \boldsymbol{w}_{N}\right]^{t}$ to be the weighting coefficient vector. By varying these weights, $\boldsymbol{\Phi}_{\mathbf{p}}$ can cover all values of the training distance functions and, hence, the shape model changes according to all of the given images [63].

\subsubsection{Segmentation method}

To estimate the initial labeling $\mathrm{f}^{*}$, the graph cuts which integrates the linear combination of Gaussian (LCG) and Markov-Gibbs random field (MGRF) model is used [61]. An example of the initial labeling is shown in Figure 5.10c. To segment vertebrae, the volume was initially labeled based on its gray level probabilistic model. Then a weighted undirected graph with vertices corresponding to the set of volume voxels $\mathcal{P}$, and a set of edges connecting these vertices is created [57]. Each edge is assigned a nonnegative weight. The graph also contains two special terminal vertices $\boldsymbol{S}$ (source) "vertebrae", and $\boldsymbol{t}$ (sink) "background". Consider a neighborhood system in $\mathcal{P}$, which is represented by a set $\mathcal{N}$ of all unordered pairs $\{p, q\}$ of neighboring voxels in $\mathcal{P}$. Let $\mathcal{L}$ the set of labels \{“0”, "1"\}, correspond to the vertebrae and background regions respectively. Labeling is a 
mapping from $\mathcal{P}$ to , and denote the set of labeling by $\mathbf{f}=\left\{\boldsymbol{f}_{\mathbf{1}}, \ldots, \boldsymbol{f}_{\boldsymbol{p}}, \ldots, \boldsymbol{f}_{|\mathcal{P}|}\right\}$. In other words, the label $f_{p}$, which is assigned to the voxel $\boldsymbol{p} \in \mathcal{P}$, segments it to vertebrae or background region. Now the goal is to find the initial segmentation, $\mathrm{f}^{*}$, by minimizing the following energy function [61]:

$$
\boldsymbol{E}\left(\mathbf{f}^{*}\right)=\sum_{p \in \mathcal{P}} \boldsymbol{D}_{\boldsymbol{p}}\left(\boldsymbol{f}_{\boldsymbol{p}}\right)+\sum_{\{p, \boldsymbol{q}\} \in \mathcal{N}} \boldsymbol{V}\left(\boldsymbol{f}_{\boldsymbol{p}}, \boldsymbol{f}_{\boldsymbol{q}}\right)
$$

$\mathrm{D}\left(f_{p}\right)$ measures how much assigning a label $f_{p}$ to voxel $p$ disagrees with the voxel intensity, $I_{p}$, and $V\left(f_{p}, f_{q}\right)$ is the pairwise interaction model which represents the penalty for the discontinuity between voxels $p$ and $q$. For more information see [57]. Initially segmented region is used to obtain the SDF $\left(\Phi_{\mathrm{f}^{*}}\right)$ which is required in the next step; see Figure 5.2d.

To use the shape prior in the segmentation process, we need to register $\mathrm{f}^{*}$ and the shape prior $\mathrm{p}$. The objective of the shape registration problem is to find the point-wise transformation between any two given shapes $\alpha$ and $\beta$ minimizing a certain energy function based on some dissimilarity measure.

In this chapter, the similar notation scheme in [65] is used. Let us define the result by $\beta$ that is obtained by applying a transformation A (with scale, rotation, and translation parameters) to a given contour/surface $\alpha$ (It is clear that $\beta$ and $\alpha$ correspond to $\mathrm{f}^{*}$ and $\mathrm{p}$ ). The shape representation used in this work changes the problem from the $2 \mathrm{D} / 3 \mathrm{D}$ shape to the higher dimensional representation. Hence, we will look for a transformation A that gives pixel-wise correspondences between the two shape representations $\Phi_{\alpha}$ and $\Phi_{\beta}$. For the $2 \mathrm{D}$ case, we assume that the transformation has scaling components, $\mathbf{S}=\left[\begin{array}{cc}\boldsymbol{s}_{\boldsymbol{x}} & \mathbf{0} \\ \mathbf{0} & \boldsymbol{s}_{\boldsymbol{y}}\end{array}\right]$, rotation angles $\mathbf{R}=\left[\begin{array}{cc}\cos (\theta) & -\sin (\theta) \\ \sin (\theta) & \cos (\theta)\end{array}\right]$, and translations represented as $\mathbf{T r}=$ 
$\left[\begin{array}{ll}\boldsymbol{T}_{x} & \boldsymbol{T}_{y}\end{array}\right]^{t}$. As discussed in Chapter 3, the transformation will be in the form $\mathrm{A}(\mathrm{x})=$ $\mathrm{SRx}+\operatorname{Tr}$. After scaling the components of the $\Phi_{\mathrm{f}^{*}}$ by A, the dissimilarity measure will be:

$$
\mathbf{r}=\|\mathbf{S}\| \boldsymbol{\Phi}_{\boldsymbol{p}}-\boldsymbol{\Phi}_{\mathrm{f} *}(\mathbf{A})
$$

(See section 3.3.1) and the squared magnitude of the above measure is summed over the image domain $\Omega$ to get an optimization energy function:

$$
E\left(\Phi_{\mathbf{p}}, \Phi_{\mathbf{f}^{*}}\right)=\int_{\Omega} \delta_{\varepsilon}\left(\Phi_{\mathbf{p}}, \Phi_{\mathbf{f}^{*}}\right) \mathbf{r}^{t} \mathbf{r} d \Omega
$$

where $\delta_{\varepsilon}$ is an indicator function defined as:

$$
\delta_{\varepsilon}\left(\Phi_{\mathrm{p}}, \Phi_{\mathrm{f}^{*}}\right)=\left\{\begin{array}{ll}
0 & \text { ifmin }\left(\left|\Phi_{\mathrm{p}}\right|,\left|\Phi_{\mathrm{f}^{*}}\right|\right)>\varepsilon \\
1 & \text { ifmin }\left(\left|\Phi_{\mathrm{p}}\right|,\left|\Phi_{\mathrm{f}^{*}}\right|\right) \leq \varepsilon
\end{array},\right.
$$

Due to $\delta_{\varepsilon}$, all pixels of a distance (measured from the nearest point on the boundary) greater than $\varepsilon$ are not considered in the energy optimization problem which reduces the computational time of the problem (Narrow-banding effect).

As discussed in chapter 3, after applying the gradient descent method, it is clear that [59]:

$$
\begin{gathered}
\frac{d}{d t} s_{i}=2 \int_{\Omega} \delta_{\varepsilon}\left(\Phi_{\mathrm{p}}, \Phi_{\mathrm{f}^{*}}\right) \mathbf{r}^{t}\left[\nabla_{s_{i}}\|S\| \Phi_{\mathbf{p}}-\nabla \Phi_{\mathrm{f}^{*}}^{t} \nabla_{s_{i}} \mathrm{~A}\right] d \Omega, \\
\frac{d}{d t} \theta_{i}=2 \int_{\Omega} \delta_{\varepsilon}\left(\Phi_{\mathrm{p}}, \Phi_{\mathrm{f}^{*}}\right) \mathbf{r}^{t}\left[\nabla \Phi_{\mathrm{p}}^{t} \nabla_{\theta_{i}} \mathrm{~A}\right] d \Omega \\
\frac{d}{d t} \mathcal{T}_{i}=2 \int_{\Omega} \delta_{\varepsilon}\left(\Phi_{\mathbf{p}^{\prime}}, \Phi_{\mathrm{f}^{*}}\right) \mathbf{r}^{t}\left[\nabla \Phi_{\mathrm{f}^{*}}^{t}\right] d \Omega
\end{gathered}
$$

where $\boldsymbol{s}_{\boldsymbol{i}} \in\left\{\boldsymbol{s}_{\boldsymbol{x}}, \boldsymbol{s}_{\boldsymbol{y}}\right\}, \quad \boldsymbol{\theta}_{\boldsymbol{i}} \in\left\{\boldsymbol{\theta}_{\boldsymbol{x}}, \boldsymbol{\theta}_{\boldsymbol{y}}\right\}$ and $\boldsymbol{T}_{\boldsymbol{i}} \in\left\{\boldsymbol{\mathcal { T }}_{\boldsymbol{x}}, \boldsymbol{\mathcal { T }}_{\boldsymbol{y}}\right\}$ of the transformation A. Regarding to the weighting coefficients $\boldsymbol{w}_{\boldsymbol{n}}$ 's (in 5.9), and similar to [59], the energy function is a quadratic function of this weights, which leads to a closed-form when the derivatives with respect to the weights are zeros: 


$$
\Psi \mathbf{w}=\Lambda
$$

where $\Lambda$ is a column vector of size $N$ and $\Psi$ is and $N \times N$ matrix. Their elements are calculated as follows [65]:

$$
\begin{aligned}
& \Lambda_{i}=\int_{\Omega} \delta_{\varepsilon}\left(\Phi_{p}, \Phi_{f^{*}}\right)\left[S \Phi_{f^{*}}-\bar{\Phi}(A)\right]^{t}\left[\widetilde{\Phi}_{i}(A)-\bar{\Phi}(A)\right] d \Omega, \\
& \left.\Psi_{i j}=\int_{\Omega} \delta_{\varepsilon}\left(\Phi_{p}, \Phi_{f^{*}}\right)\right)\left[\widetilde{\Phi}_{j}(A)-\bar{\Phi}(A)\right]^{t}\left[\widetilde{\Phi}_{i}(A)-\bar{\Phi}(A)\right] d \Omega,
\end{aligned}
$$

$\forall(i, j) \in[1, N] \times[1, N]$. Using unique training shapes (with variabilities not identical) guarantees that $\Psi$ is a positive definite matrix avoiding singularity.

\subsubsection{Experimental Results}

The proposed framework is applied on clinical CT spine bone images. The clinical datasets were scanned at $120 \mathrm{kV}$ and $3.0 \mathrm{~mm}, 2.5 \mathrm{~mm}, 1.33 \mathrm{~mm}$, or $0.67 \mathrm{~mm}$ slice thickness. The algorithm was tested on $500 \mathrm{CT}$ slices/25 VBs which are obtained from 15 different patients. The goal is to segment the VB region correctly. The segmentation accuracy and robustness of the framework are tested on the phantom named as the European Spine Phantom (ESP) as well as the clinical datasets. All algorithms are implemented using $\operatorname{Matlab}{ }^{2}$.

To assess the proposed method under various challenges, a zero mean Gaussian noise with different signal-to-noise ratios (SNR) - from $0 \mathrm{~dB}$ to $100 \mathrm{~dB}$ - was added to the CT images. The segmentation accuracy is measured for each method using the ground truths. It should be noted that the ground truths are validated by a radiologist. The percentage segmentation accuracy $(A c c)$ is calculated as follows:

\footnotetext{
${ }^{2}$ All algorithms are run on a PC with a $2 \mathrm{GHz}$ Core i7 Quad processor with 6GB RAM.
} 


$$
\text { Acc } \%=100 *\left(1-\frac{F P+F N}{\text { The tota mumber of slice pixels }}\right)
$$

where $F P$ represents the false positive (i.e. the total number of the misclassified pixels of the background), and $F N$ is the false negative (i.e. the total number of the misclassified pixels of the object).

A variety of methods was adopted to measure the accuracy of this framework. First, the visual inspection was used to evaluate the segmentation quality of the approach. Figure 5.12 compares the results of different examples for the initial segmentation step using the scalar level set model [64] and the graph cut method [61] which is used in the proposed framework. As shown in this figure, the scalar level sets method fails to segment the whole vertebra in many cases. However, the graph cut approach can segment them well. Additionally, the boundaries detected by scalar level sets are not smooth, and some obvious boundaries are not detected. The graph cut method segments the image accurately. Figure 5.13 shows various segmentation results of three different methods applied on some clinical datasets. These methods are: i) The graph cut segmentation (identical to initial labeling in this algorithm), ii) The PCA based segmentation described in [58], and iii) The 2D-PCA based tensor level segmentation. The segmentation accuracies of the 2D-PCA based results shown in row (iii) are: $96.8 \%, 92.6 \%, 91.2 \%$ and $93.6 \%$ respectively. For PCA based results in row (ii), the segmentation accuracies are: $89.3 \%, 87.4 \%, 85.6 \%$, and $84.5 \%$ respectively. It is clear that this method is more accurate than the method in [58]. Figure 5.14 shows the segmentation results of the ESP using (i) graph cut method and (ii) the segmentation algorithm (graph cut + shape prior) under different noise level. With the proposed approach, much better results are obtained compared to the graph cut only. Figure 
5.15 studies the effect of the initialization on the proposed framework. Results indicate that the performance of this method is almost constant with different initialization parameters. To quantitatively demonstrate the accuracy of the approach, the average segmentation accuracy of the segmentation method on $500 \mathrm{CT}$ images under various signal-to-noise ratios is calculated and the results are compared with the PCA based segmentation method in [58]. Again, as mentioned before, the 2D-PCA based framework outperforms the conventional PCA described in [58] as shown in Figure 5.16a. Additionally, Figure 5.16b studies the effect of choosing the number of the projected training shapes $N$ (see section 5.5.1) on the segmentation accuracy. From this figure, it can be concluded that the performance of 2D-PCA is better than the conventional PCA under the same number of training shapes. In another word, to get the same accuracy of PCA framework, the 2D-PCA needs fewer training shapes.

As a summary for this approach, a new shape based segmentation of VBs is proposed in clinical CT images using 2D-PCA. Validity was analyzed using ground truths of clinical datasets as well as the European Spine Phantom (ESP). The experimental results show that the noise immunity and the segmentation accuracy of 2D-PCA based approach are much higher than conventional PCA approach. On the other hand, the proposed system is fully automatic where it does not need any user interaction. 


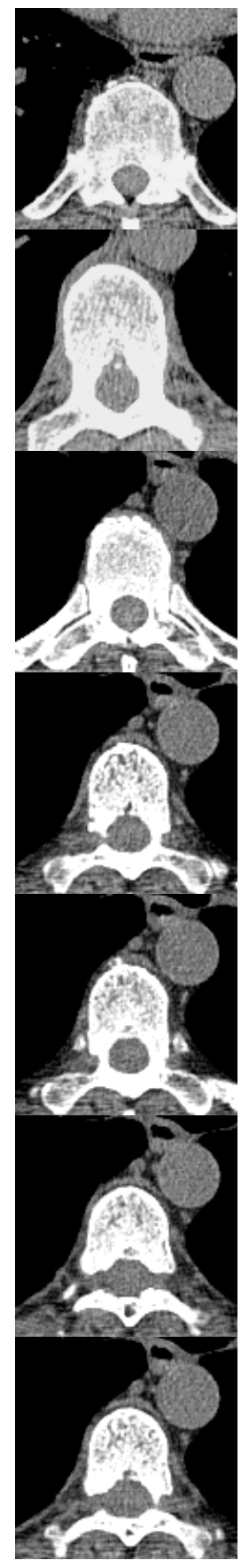

(a)

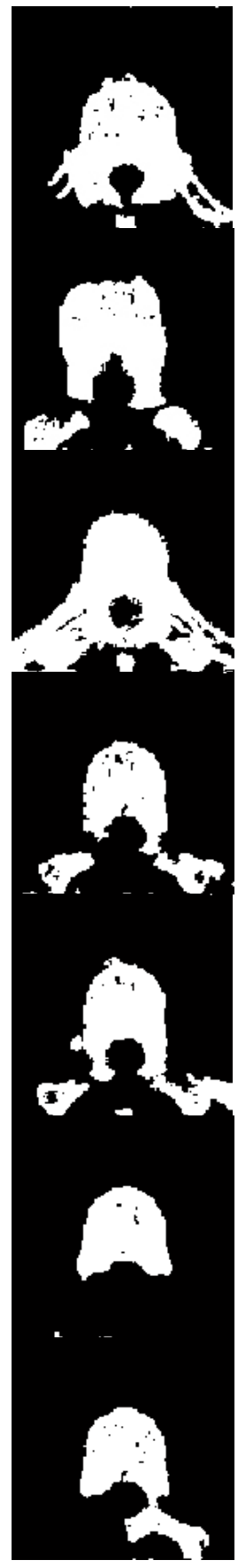

(b)

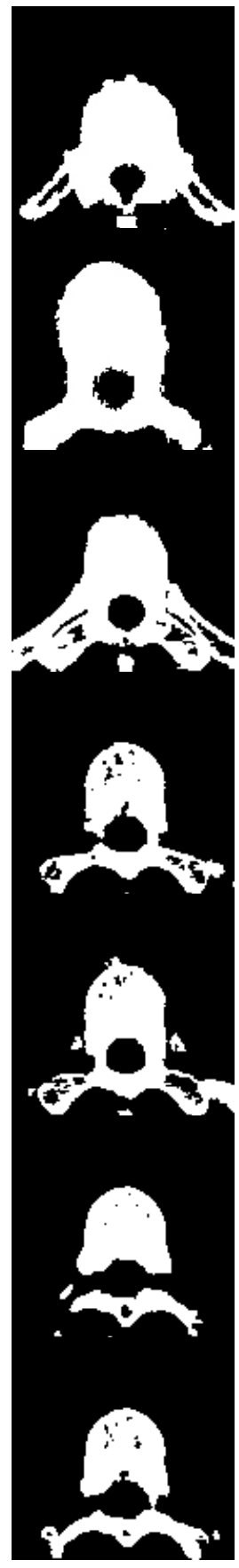

(c)

Figure 5.12. Comparison between the intensity based segmentation (initial labeling) using: (b) Scalar level sets model [64], and (c) graph cut method [61]. 

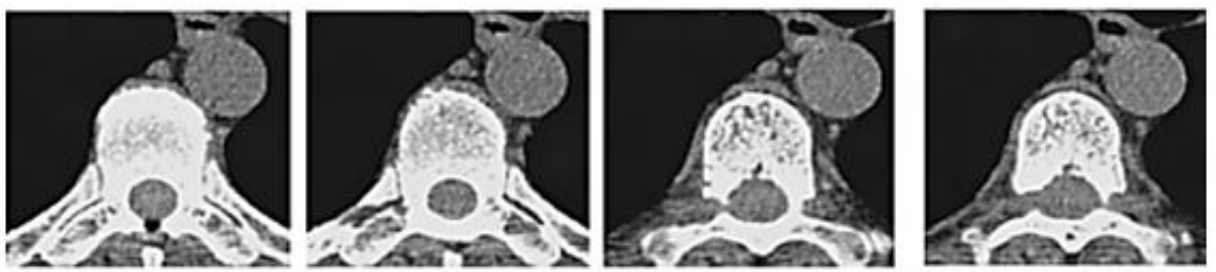

(i)
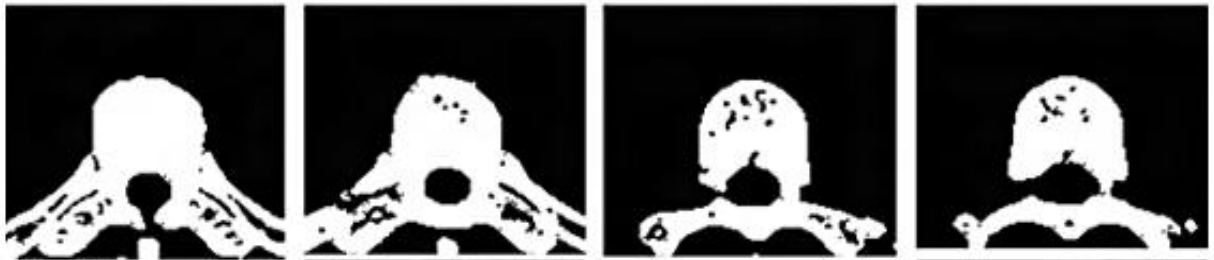

(ii)
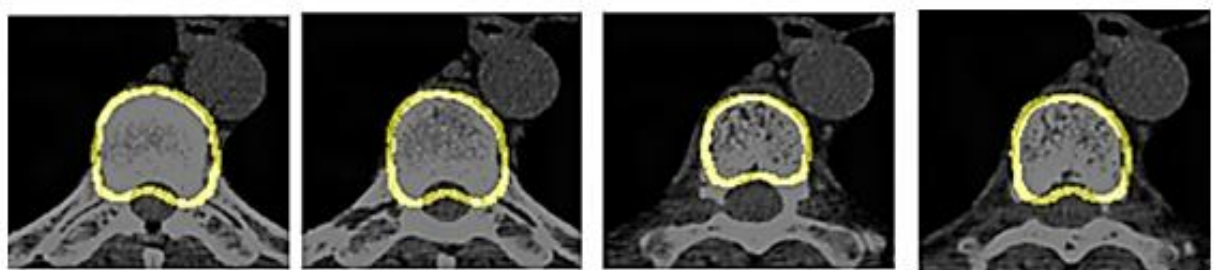

(iii)
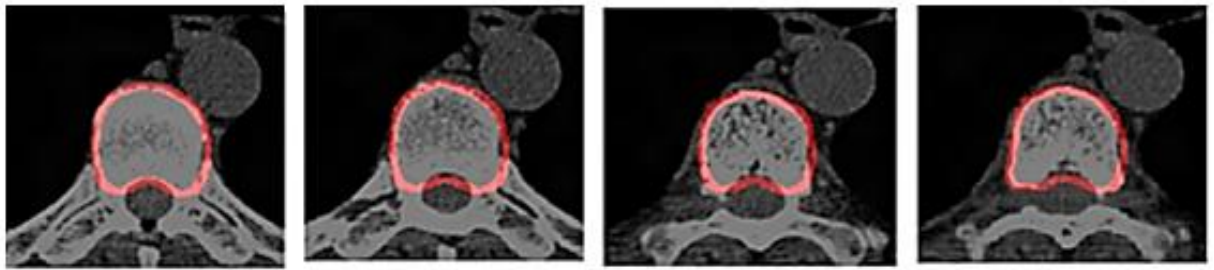

Figure5.13. Segmentation results of three different methods: (i) using graph cuts only, (ii) Method described in [58], and (iii) the 2D-PCA based segmentation.

(i)
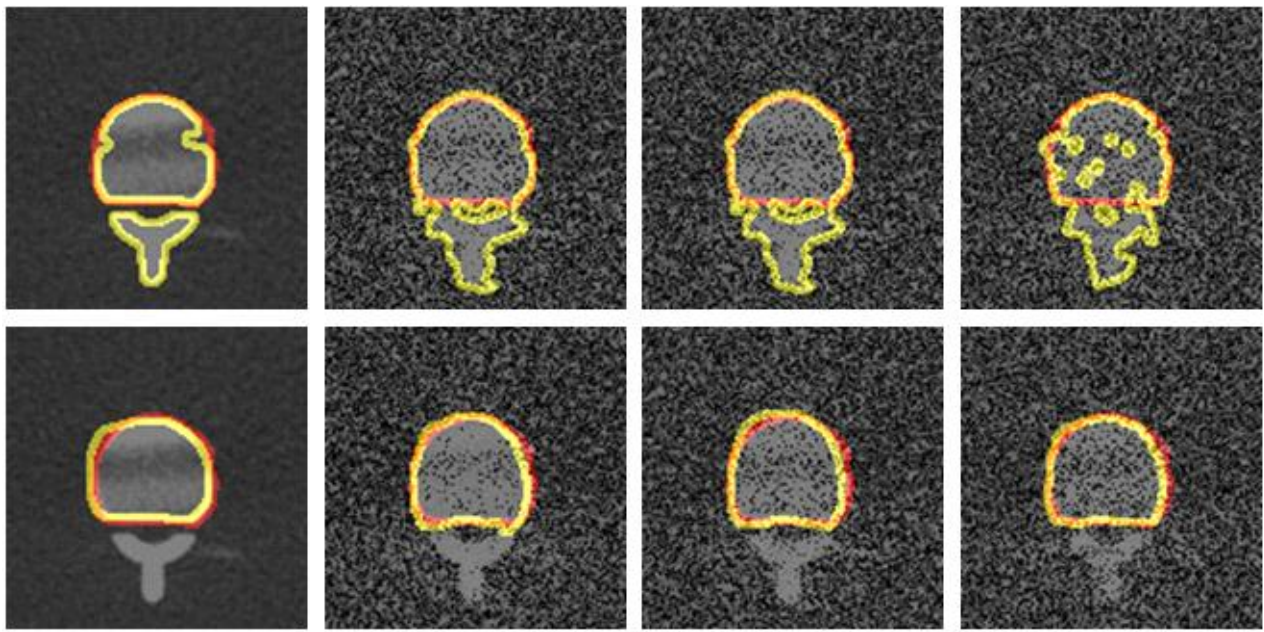

Figure5.14. Segmentation results of the ESP under different noise levels (i) using graph cut only. (ii) The algorithm (graph cut + shape prior). The red and yellow colors show the contour of the gold standards and segmented regions. 

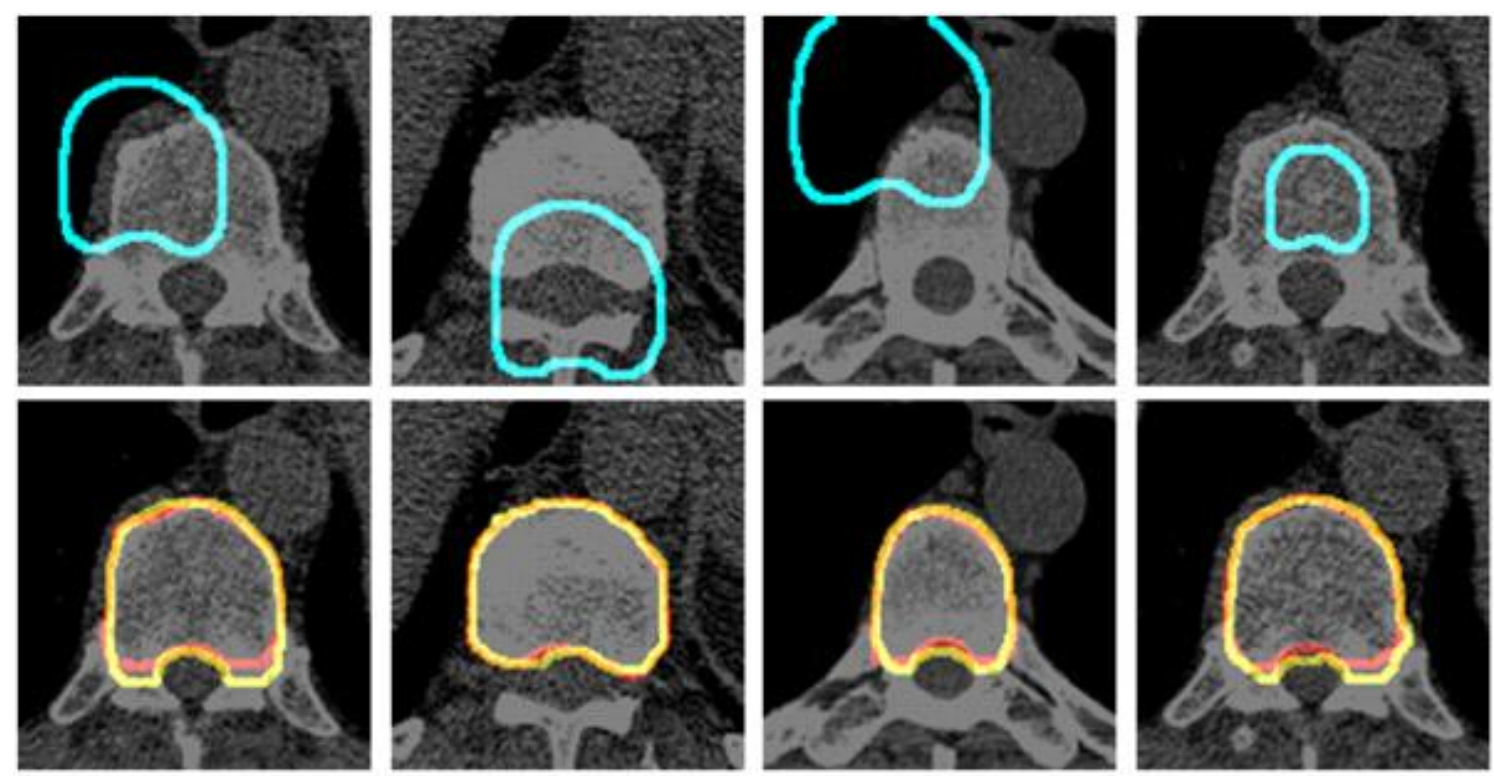

Figure5.15. Segmentation results with various shape initialization. (i) the initial shape prior, and (ii) is the final results. The red and yellow colors show the contour of the gold standards and segmented regions.

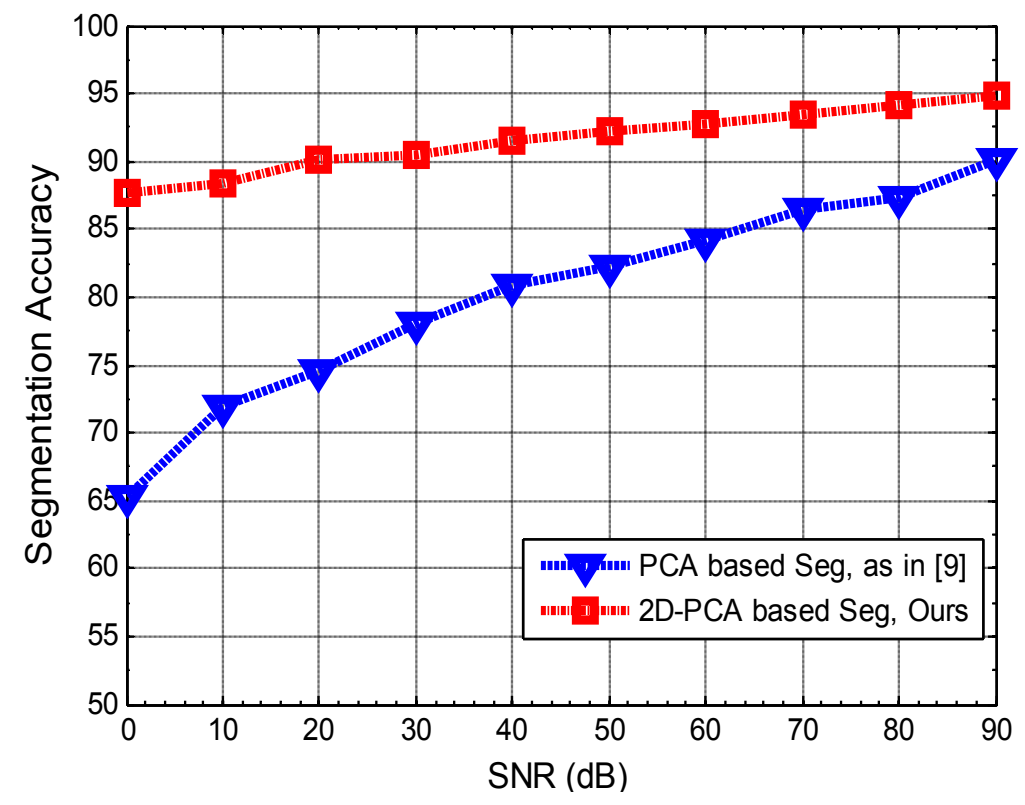

(a) 


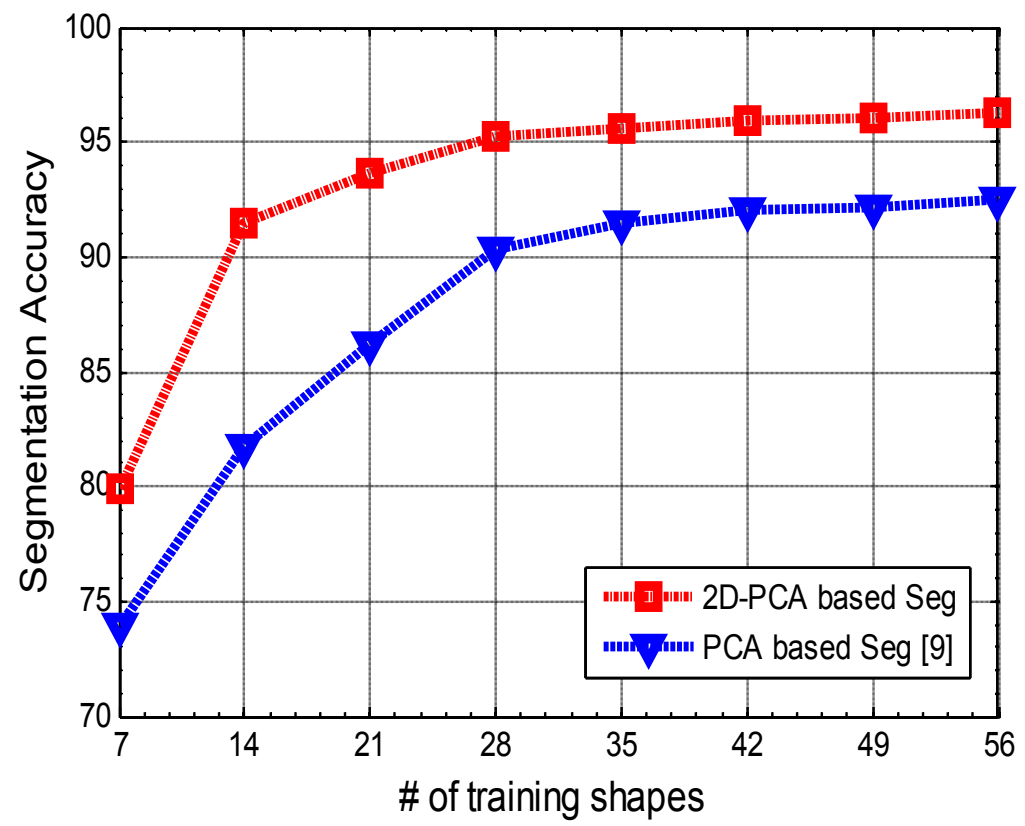

(b)

Figure 5.16. (a) The average segmentation accuracy of different segmentation methods on $500 \mathrm{CT}$ images under various signal-to-noise ratios.(b) The effect of choosing the number of the projected training shapes $N$ on the segmentation accuracy.

\subsection{Three Dimensional Approach for VB Segmentation}

In this approach, a 3D shape based iterative segmentation and registration method is introduced. Figure 5.17 summarizes the main components of the proposed framework. This framework contains two phases. In the first phase, a probabilistic shape model is obtained as previously presented in [61]. Shape information is gathered from a set of training shapes. Then the shape variations are estimated using a new distance probabilistic model which approximates the marginal densities of the vertebral body and its background in the variability region. In the second phase, we initialize a disk as the initial evolving front on the VB volume. Then, an iterative process which simultaneously does the segmentation and registration begins. 


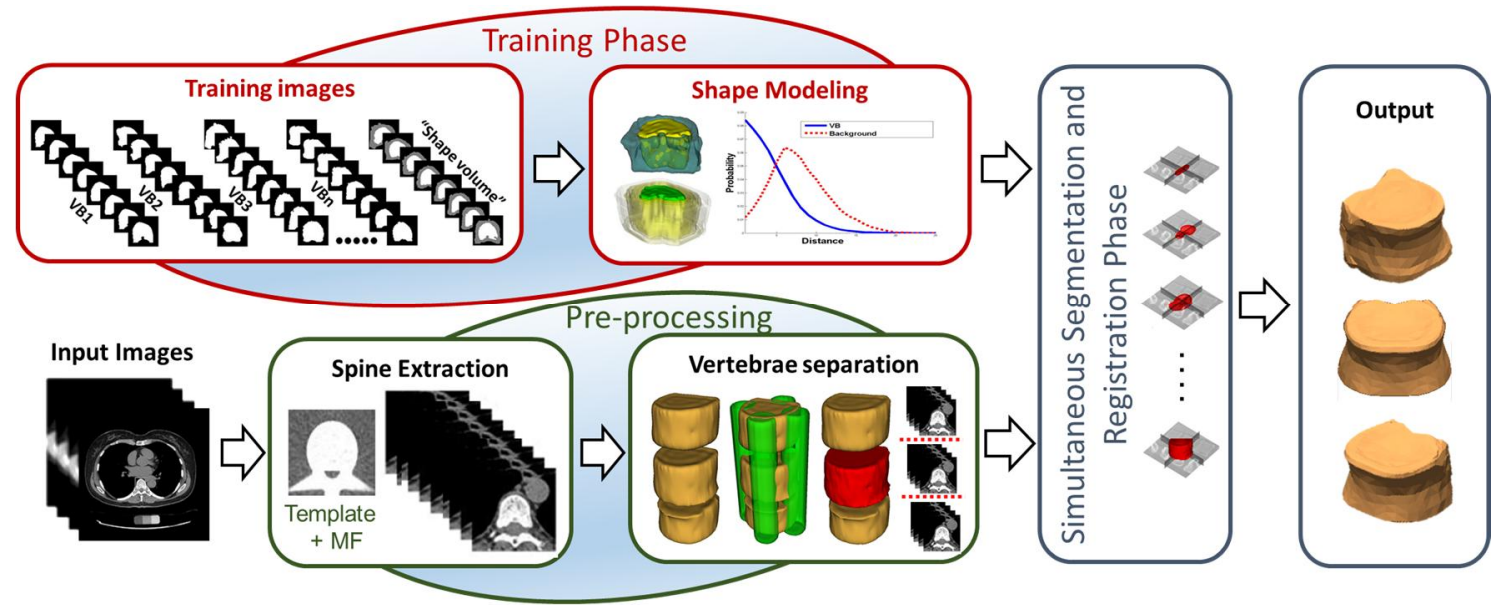

Figure 5.17. Block diagram of the proposed simultaneous segmentation and registration method for human vertebral bodies (VB) extraction from CT images. This framework contains two main components; the training phase and the segmentation phase. A pre-processing step is used to eliminate the user interaction and reduce the execution time.

As discussed before, the ROI of the input CT images is detected and cropped using the MF. The benefits of the preprocessing step are twofold: 1) it eliminates the user interaction; 2) it improves the segmentation accuracy and minimizes the execution time.

In the segmentation step, an improved level sets approach is used based on the edgemounted Willmore flow (adopted in [120]) in which a probabilistic shape model is integrated. To make the shape prior to be invariant to the transformation, we register it to the evolving front at each iteration. The overall segmentation framework is given in Algorithm 1. The following sections give more details about the shape model construction and the proposed method.

\subsubsection{Training and Shape Modeling}

A $3 \mathrm{D}$ shape of vertebral body is created from a subset of VB data sets. These VBs are selected from 10 healthy and 10 with low bone mass patients. This is done as follows: 30 
VBs' volumes, where each VB consists of $8 \mathrm{CT}$ slices, are manually segmented by a medical expert. Then the segmented binary images are aligned together using the $2 \mathrm{D}$ registration described in [56]. Finally, a "shape volume" $\mathcal{P}_{\boldsymbol{s}}=\mathcal{O} \cup \mathcal{B} \cup \mathcal{V}$ is generated, which its slices are shown in Fig 5.18. Three regions in this shape model: white color represents the object region $\mathcal{O}(\mathrm{VB})$, black represents $\mathcal{B}$ (its background), and gray is the variability region $\mathcal{V}$. Fig. 5.19(a) illustrates a 3D view of the VB and its variability region.

To model variability region $\mathcal{V}$, we use a distance probabilistic model to capture the $3 \mathrm{D}$ shape variations [61]. The distance probabilistic model describes the VB (and background) in the variability region as a function of the following normal distance.

$$
d_{p}=\min _{c \in \mathbf{C}_{o v}}\|p-c\|
$$

from a voxel $\boldsymbol{p} \in \boldsymbol{V}$ to the organ/variability surface $\mathbf{C}_{\mathcal{O V}}$. Each set of voxels located at equal distance $\boldsymbol{d}_{\boldsymbol{p}}$ from $\mathbf{C}_{\boldsymbol{O} v}$ constitutes an iso-surface $\mathbf{C}_{\boldsymbol{d}_{\boldsymbol{p}}}$ for $\mathbf{C}_{\mathcal{O V}}$ as shown in Figure 5.19(b). To estimate the marginal density of the vertebral body, it is assumed that each iso-surface $\mathbf{C}_{\boldsymbol{d}_{\boldsymbol{p}}}$ is a normally propagated wave from $\mathbf{C}_{\boldsymbol{o v}}$. The probability of an iso-surface to be object decays exponentially as the discrete index $\boldsymbol{d}_{\boldsymbol{p}}$ increases. So we model the distance histogram by a Poisson distribution. We estimate the vertebral body's distance histogram as follows. The histogram entity at distance $\boldsymbol{d}_{\boldsymbol{p}}$ is defined as

$$
\boldsymbol{h}_{d p}=\sum_{i=1}^{M} \sum_{j=1}^{K} \sum_{p \in \mathbf{C}_{d p}} \delta\left(p \in \mathcal{O}_{i j}\right)
$$

(where the indicator function $\delta(\mathrm{A})$ equals 1 when the condition $\mathrm{A}$ is true, and zero otherwise, $M(=30)$ is the number of training data sets, $K(=8)$ is the number of CT slices of each data set, and $\boldsymbol{O}_{\boldsymbol{i} \boldsymbol{j}}$ is the vertebral body region in the training set $i$ and in the slice $j$. 


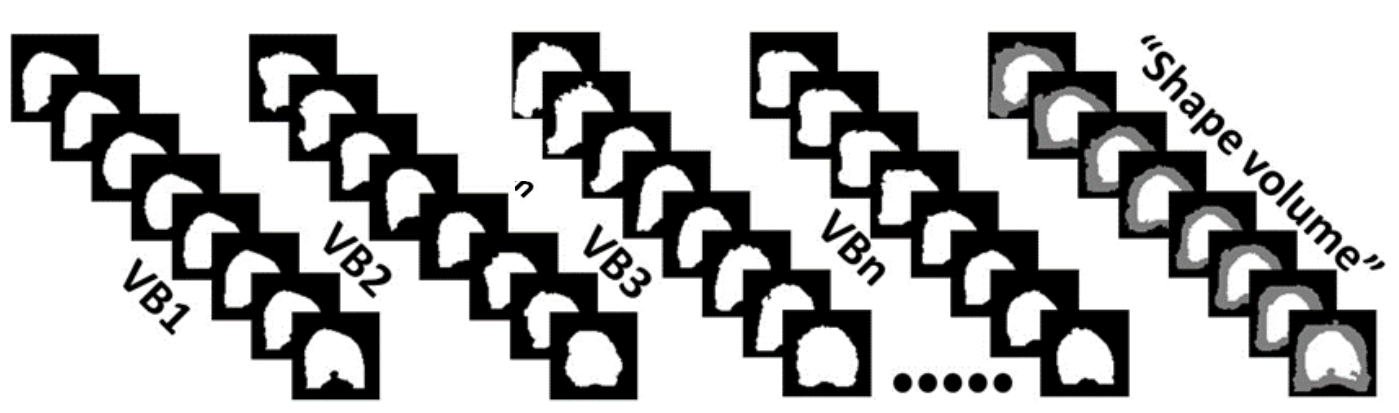

Figure 5.18. Constructing the shape prior volume. $\left\{\mathrm{VB}_{1}, \cdots, \mathrm{VB}_{M}\right\}$ training $\mathrm{CT}$ slices of different data sets. ( $M$ represents the number of training data sets). Last column shows the shape prior slices with variability region.
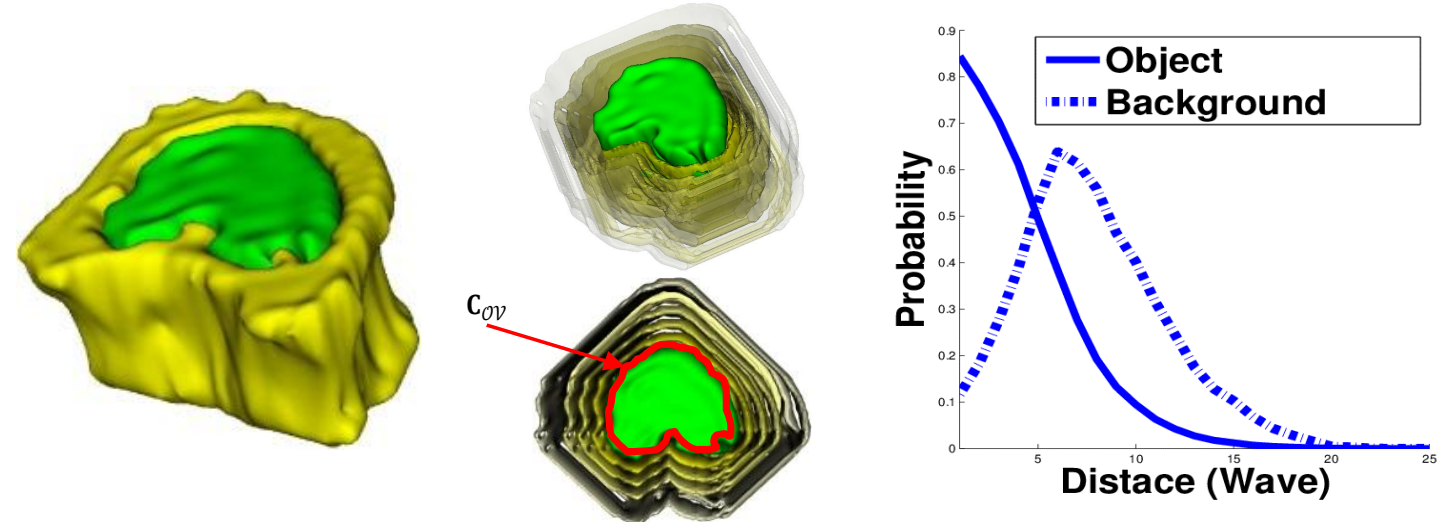

Figure 5.19 (a) A 3D view of the 3D shape prior. (b) Different 3D views for the iso-surfaces $\mathbf{C}_{d_{p}}, p \in \mathcal{V}$. Green color represents the object region $\mathcal{O}$, yellow color is the variability region $\mathcal{V}$, gray waves represent the the iso-surfaces $\mathbf{C}_{d_{p}}$, and red contour is the object/variability surface $\mathbf{C}_{\mathcal{O V}}$. (c) The probability of the object and background in the variability region.

We change the distance $\boldsymbol{d}_{\boldsymbol{p}}$ until we cover the whole distance domain available in the variability region. Then we multiply the histogram with vertebral body prior value, which is defined as follows:

$$
\pi_{\mathcal{O}}=\frac{1}{M K|\mathcal{V}|} \sum_{i=1}^{M} \sum_{j=1}^{K} \sum_{p \in \mathcal{V}} \delta\left(p \in \mathcal{O}_{i j}\right)
$$

Finally, we calculate the distance marginal density of the object region as:

$$
P_{\mathcal{O}}\left(d_{p}\right)=h_{d p} \pi_{\mathcal{O}} / M K\left|\mathrm{C}_{d_{p}}\right|
$$

The same scenario is repeated to obtain the marginal density of the background. An example of the distance marginal densities of the object and background region is shown in Figure 5.19(c). 


\subsubsection{Simultaneous Segmentation and Registration}

The level set method has been widely used for medical image segmentation. It achieved good results when coupled with prior shape models [55]. The level set segmentation framework contains a moving front, denoted by $\mathcal{C}$, which is implicitly represented by the zero level of a higher dimensional function, $\boldsymbol{\phi}$, that is: $\mathcal{C}(\boldsymbol{t})=\{\boldsymbol{p} / \boldsymbol{\phi}(\boldsymbol{p}, \boldsymbol{t})=\mathbf{0}\}$. The equation that governs the evolution of the level set function $\boldsymbol{\phi}$ is $\partial \phi / \partial t+F|\nabla \boldsymbol{\phi}|=0$, where $F$ represents the speed function. In more recent applications, the variational framework is often considered. Under the variational framework, an energy $\boldsymbol{E}(\boldsymbol{\phi})$ is defined in relation to the speed function, and minimization of the energy generates the Euler-Lagrange equation and, hence, providing the evolution equation through the gradient descent as:

$$
\frac{\partial \phi}{\partial t}=-\frac{\partial E(\phi)}{\partial \phi}
$$

In this work, the energy function of the segmentation can be formulated as

$$
E(\phi)=E_{\text {intensity }}(\phi)+\alpha E_{\text {sha }} \text { pe }(\phi),
$$

where $\alpha$ is a constant which controls how much we depend on the probabilistic shape prior. The first energy term is based on the intensity of the testing volume. The second term is based on the shape prior after registering it to the evolving front to be invariant to the

transformation parameters. More details about $\boldsymbol{E}_{\text {intensity }}$ and $\boldsymbol{E}_{\boldsymbol{s h a}} \boldsymbol{p} \boldsymbol{e}^{\text {will be described in }}$ the following sections.

\subsubsection{Intensity information}

Willmore energy is a function of mean curvature, which is a quantitative measure of how much a given surface deviates from a round sphere. It has been applied to image inpainting, 
restoration of implicit surfaces [116], [118], and to studies of the bending energy of biological cell membranes as these cell membranes tend to position themselves to minimize Willmore energy [119]. Willmore flow is the gradient flow of Willmore energy. Willmore flow of a surface is the evolution of the surface in time to follow variations of the Willmore energy. Willmore energy was defined after the British Geometer T. Willmore [119] and is formulated as

$$
E_{\omega}=\frac{1}{2} \int_{\Gamma} h^{2} d A
$$

where $\boldsymbol{\Gamma}$ is a d-dimensional surface embedded in $\mathbb{R}^{\mathrm{d}+1}$ and $h$ the mean curvature on $\boldsymbol{\Gamma}$.

In this method, we integrate Willmore flow into the level set segmentation framework as a geometric functional. Willmore energy is defined on the collection of level sets, and Willmore flow is enabled by defining a suitable metric, the Frobenius norm, on the space of the level sets. The Frobenius norm of an arbitrary matrix $A=\left(a_{i j}\right)_{k \times z}$, which is defined as $\|\boldsymbol{A}\|_{F}=\left(\sum_{i=1}^{k} \sum_{j=1}^{z}\left|\boldsymbol{a}_{i j}\right|^{\mathbf{2}}\right)^{\mathbf{1} / \mathbf{2}}$, coincides with the calculation for the gradient descent. It is equivalent to the $l^{2}$-norm (the Euclidean norm) of a matrix, More importantly, it is computationally attainable comparing to $l^{2}$-norm. As Frobenius norm is an inner-product norm, the optimization in the variational method comes naturally. Based on the formulation by Droske and Rumpf [120], Willmore flow or the variational form for the Willmore energy with respect to $\boldsymbol{\phi}$ is

$$
\frac{\partial E_{\omega}}{\partial \phi}=-|\nabla \phi|\left(\Delta_{\Gamma} h+h\left\{\|S\|^{2}-\frac{1}{2} h^{2}\right\}\right)
$$


where $\Delta_{\Gamma} \boldsymbol{h}=\Delta \boldsymbol{h}-\boldsymbol{h} \frac{\boldsymbol{\partial} \boldsymbol{h}}{\boldsymbol{\partial n}}-\frac{\boldsymbol{\partial}^{2} \boldsymbol{h}}{\partial \boldsymbol{n}^{2}}$ is the Laplacian Beltrami operator on $h$ with $n=\frac{\boldsymbol{\nabla} \boldsymbol{\phi}}{|\boldsymbol{\nabla} \phi|}, S=(\mathrm{I}$ $-n \otimes n)(\nabla \times \nabla) \boldsymbol{\phi}$ is the shape operator on $\boldsymbol{\phi}$, and $\|\boldsymbol{S}\|$ is the Frobenius norm of $S$.

In order to ensure that the smoothing effect of Willmore energy acts around the constructed surface and does not affect adversely the edge of vertebrae, we multiply the

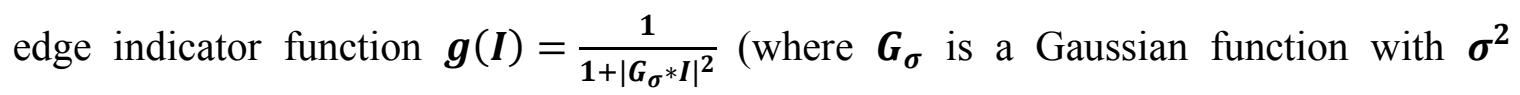
variance) to the level set evolution[20]:

$$
\frac{\partial E_{\text {intensity }}}{\partial \phi}=-g(I)|\nabla \phi|\left(\Delta_{\Gamma} h+h\left\{\|S\|^{2}-\frac{1}{2} h^{2}\right\}\right)
$$

\subsubsection{Embedding shape prior information}

In this work, our contribution is to propose a new probabilistic energy function in the level set method using previously presented shape model [61]. To register the shape model to the evolving front, we use the similar approach presented in [56]. Each voxel in the shape prior has two probabilities for being $i$ ) an object and ii) a non-object. The shape prior is embedded in the level sets function in order to obtain more accurate segmentation results and extract the spinal processes automatically. The shape model is registered into the volume domain $\boldsymbol{\Omega}$ by maximizing the probability of voxels inside the front belonging to the object space and the voxels outside the front belonging to the non-object space. This approach leads to the following energy function:

$$
\begin{aligned}
E_{\text {sha } p \mathrm{e}}(\phi)=\int_{\Omega}\left(1-\boldsymbol{P}_{\mathcal{O}}\left(\sigma_{\phi} d_{p}+\mu_{\phi}\right)\right) H_{s} \phi(p) d p & \\
& +\int_{\Omega}\left(1-P_{\mathcal{B}}\left(\sigma_{\phi} d_{p}+\mu_{\phi}\right)\right)\left(1-H_{s} \phi(p)\right) d p,
\end{aligned}
$$

where $\phi$ represents the signed distance function of the evolving front, $H_{s}$ is the Heaviside step function, and the translation and scaling parameters can be estimated as: 


$$
\begin{gathered}
\mu_{\phi}=\frac{\int_{\Omega} p H_{s} \phi(p) d p}{\int_{\Omega} H_{s} \phi(p) d p}, \\
\sigma_{\phi}^{2}=\frac{\left.\int_{\Omega}\left(p-\mu_{\phi}\right)^{2} H_{s} \phi(p) d p\right)}{\int_{\Omega} H_{s} \phi(p) d p} .
\end{gathered}
$$

After applying the gradient descent method, the gradient of this energy terms will be:

$$
\frac{\partial E_{\text {sha } p e}}{\partial \phi}=\delta(\phi)\left[P_{\mathcal{O}}\left(\sigma_{\phi} d_{p}+\mu_{\phi}\right)-P_{\mathcal{B}}\left(\sigma_{\phi} d_{p}+\mu_{\phi}\right)\right]
$$

Finally, the change of the level set function with time using the two energy function will be:

$$
\begin{aligned}
\frac{\partial \phi}{\partial t}=-\frac{\partial E_{\text {intensity }}}{\partial \phi}-\alpha \frac{\partial E_{\text {sha } p e}}{\partial \phi} & \\
=g(I)|\nabla \phi|\left(\Delta_{\Gamma} h+h\left\{\|S\|^{2}-\frac{1}{2} h^{2}\right\}\right) & -\alpha \delta(\phi)\left[P_{\mathcal{O}}\left(\sigma_{\phi} d_{p}+\mu_{\phi}\right)-\right. \\
& \left.P_{\mathcal{B}}\left(\sigma_{\phi} d_{p}+\mu_{\phi}\right)\right] .
\end{aligned}
$$

\subsubsection{Experimental Results}

In this work, the training and testing images were acquired from GE LightSpeed VCT, Toshiba Aquilion, and Imatron C-150 CT scanners with the resolution range of $0.63-0.98$ $\mathrm{mm}$ and a slice thickness of $0.63-3.00 \mathrm{~mm}$. For the testing stage, 40 data sets, 18 female and 22 male, and a phantom named as the European Spine Phantom (ESP) are examined in this study. The number of visible VBs for each scan changes from 2-7 with $16-140$ axial slices of 512x512 voxels. Totally, the proposed framework is tested on 153 of human lumbar and thoracic VBs. The ground truths are obtained by manual segmentation using a commercial 3D image segmentation software and are verified by three radiologists. 
To evaluate the results we calculate the percentage segmentation accuracy from the ground truth using the Dice's coefficient (DC) [112] and the Hausdorff distance (HD) [1]. The DC measures the concordance between two enclosed volumes as follows

$$
D C \%=100 \frac{2 T P}{F P+2 T P+F N},
$$

where $F P$ represents the number of false positive (i.e. the total number of the misclassified voxels of the background), $F N$ is the number of false negative (i.e. the total number of the misclassified voxels of the object), and $T P$ is the true positive (i.e. total number of the correctly classified pixels), as shown in Figure5.20(a).

On the other hand, The HD is defined as:

$$
H D(X, Y)=\max \left\{\operatorname{su}_{\ell \in X} \inf _{y \in Y} d(x, y), \operatorname{su~}_{\mathbf{B}_{\in Y}} \inf _{x \in X} d(x, y)\right\}
$$

where $X$ and $Y$ are the boundaries of two different volumes. It measures how far two subsets of a metric space are from each other, as shown in Figure5.20(b). A high DC and a low HD are desirable for good segmentation.

To compare the proposed method (A1) with other alternatives, VBs are subsequently segmented using: (A2) the graph cuts with shape constrained (The approach described in section 5.5), (A3) the active appearance model (AAM) methods [18], (A4) Chan-Vese models combined with same prior shape energies (CVIP work described in [61]), and (A5) the level set method implemented around a narrow band without re-initialization [50]. All algorithms are run on a PC with $2.6 \mathrm{GHz}$ Core i7 Quad processor, and 8GB RAM. Algorithms are implemented using MATLAB ${ }^{\circledR}$. 


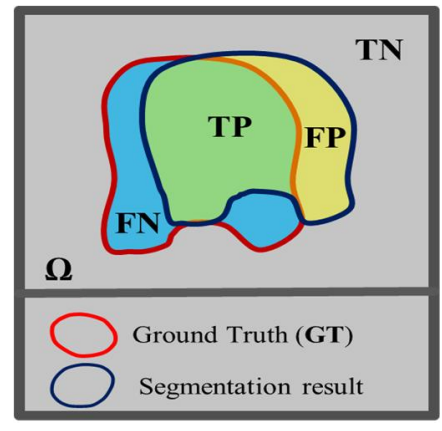

(a)

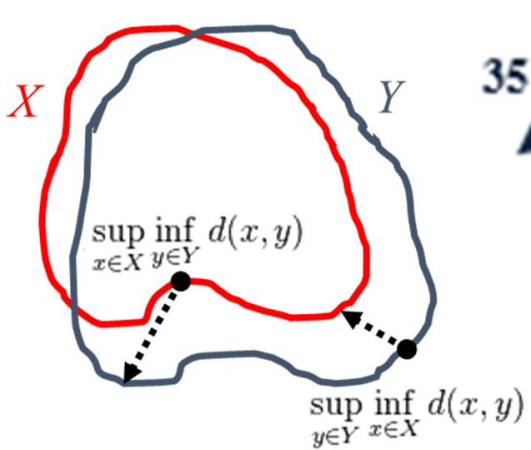

(b)

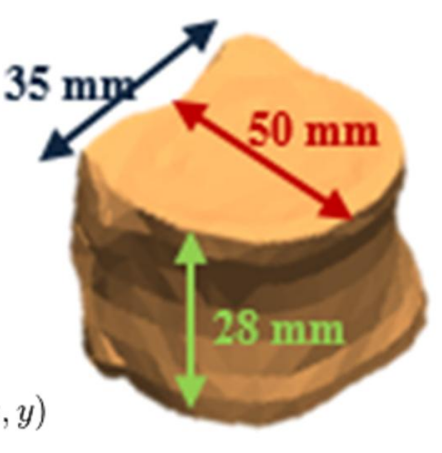

(c)

Figure 5.20. (a) In the segmentation quality measurements, there are 4 regions to be considered as: True positive (TP), false positive (FP), true negative (TN), and false negative (FN). (b) The calculation of the HD between the red line $\mathrm{X}$ and the blue line Y. (c) Average geometrical dimensions of the lumbar vertebrae, all measurements in millimeters $(\mathrm{mm})$.

\subsubsection{Results on Clinical CT Images}

The proposed algorithm is tested on 40 patients' data sets containing $153 \mathrm{VBs}$. We compare the segmentation results of our method with the four algorithms described before, i.e. A2, A3 [18], A4 [61], and A5 [50]. There is a crucial point which we need to clarify carefully. The alternative methods are tested after the preprocessing steps (spinal cord extraction and VB separation) give their output. Hence, we do not take advantage of the preprocessing steps for only the proposed segmentation process.

Table 5.1 summarizes the average segmentation accuracy (the Dice's coefficient (DC) and the Hausdorff distance (HD) in $\mathrm{mm}$ ), as well as the average execution time (in sec) for each method. The proposed method reaches $92.12 \%$ overall DC. It outperforms all other alternative. For more meaningful comparison using HD, Figure5.20(c) represents the average geometrical dimensions of the human vertebrae as described in [105]. As can be seen, the framework leads to superior results over other methods. It reaches $9.11 \mathrm{~mm}$ overall HD which reflects how accurate the proposed segmentation approach is. The experimental results in Table 5.1 show that the performance of this algorithm is superior 
in terms of accuracy over others algorithms. This improvement in accuracy leads to more execution time. There is a tradeoff between the segmentation accuracy and execution time. We believe that the improvement of segmentation accuracy is more important to obtain better BMD measurements.

Figure 5.21 illustrates the $2 \mathrm{D}$ projection in the axial view of the $3 \mathrm{D}$ level set evolution and segmentation of three different vertebra samples using the proposed method. We initialize a disk as the initial evolving surface on the VB volume. Then, an iterative process which simultaneously does the segmentation and registration begins. The resultant 3D volume of the VBs are shown in Fig. 5.21 (d). Figure 5.22 demonstrates the 2D projections of final 3D segmentation results in sagittal, coronal and axial view for different examples of the clinical data sets. These data sets have the lumbar and thoracic sections. Note that the unnecessary regions such as ribs and processes are eliminated as much as possible.

For more illustration, samples of 3D segmentation results of a clinical data set for all tested methods are shown in Figure 5.23 In this figure, the red color represents the misclassified voxels. The proposed approach is successful in extracting the VB region. Other alternative methods have lower segmentation accuracy which may change the BMD measurements.

\subsubsection{Validation Using the Phantom}

To evaluate this algorithm, we segment the European Spine Phantom (ESP), which is an accepted standard for quality control in bone densitometry [54]. The ESP dataset was scanned at $120 \mathrm{kVp}$ and $0.75 \mathrm{~mm}$ slice thickness. To assess the proposed method under various challenges, a Gaussian noise with zero mean and different variance $\sigma_{n}^{2}$ values 
(from $0,0.125,0.25$ and 0.5 ) is added to the $\mathrm{CT}$ images.

Figure 5.24 shows that the approach accurately segments the VB without its processes and compares it with other 3 alternative methods (A2, A4 and A5 respectively). The red color represents the misclassified voxels. It is clear that this algorithm outperforms other methods with average DC of $93.73 \pm 1.86 \%$. To evaluate the robustness of the proposed approach against noise, we calculate the average DC of this segmentation method on ESP dataset under various noise level and compare the results with A2, A3, A4 and A5. Again, the proposed framework outperforms the other alternatives as shown in Figure 5.25.

Table 5.1. Average DC (\%) and HD ( $\mathrm{mm})$ with standard deviation for segmentations of the clinical datasets using different methods. the average execution time of each method is summarized in the last row.

\begin{tabular}{cccccc}
\hline \hline Method & $\begin{array}{c}\text { A1 } \\
\text { PrOPOSED }\end{array}$ & $\begin{array}{c}\text { A2 } \\
\text { GC+ SHAPE }\end{array}$ & $\begin{array}{c}\text { A3 } \\
\text { AAM }\end{array}$ & $\begin{array}{c}\text { A4 } \\
\text { CV+SHAPE }\end{array}$ & $\begin{array}{c}\text { A5 } \\
\text { LS }\end{array}$ \\
\hline DC $(\%)$ & $\mathbf{9 2 . 1 2} \pm \mathbf{2 . 0 3}$ & $84.72 \pm 3.32$ & $81.97 \pm 7.05$ & $83.15 \pm 4.67$ & $50.87 \pm 8.53$ \\
\hline HD $(\mathrm{mm})$ & $\mathbf{9 . 1 1} \pm \mathbf{1 . 5 1}$ & $13.47 \pm 1.06$ & $15.03 \pm 2.37$ & $14.61 \pm 1.62$ & $24.67 \pm 3.42$ \\
\hline Avg. Time(sec) & 131.3 & $\mathbf{5 8 . 9}$ & $\mathbf{7 4 . 5}$ & 102.4 & 98.7 \\
\hline \hline
\end{tabular}

Table 5.2. Average Relative Errors in BMD Measurement using different segmentation methods

\begin{tabular}{c|c|c|c|c|c|}
\cline { 2 - 6 } & $\mathbf{A 1}$ & $\mathbf{A 2}$ & $\mathbf{A 3}$ & $\mathbf{A 4}$ & $\mathbf{A 5}$ \\
\hline Max (\%, mg/cc) & $\mathbf{5 . 9 2}$ & 9.92 & 23.6 & 17.6 & 28.4 \\
\hline Min (\%, mg/cc) & $\mathbf{0 . 0 3}$ & 0.09 & 1.89 & 1.83 & 2.35 \\
\hline Mean(\%, mg/cc) & $\mathbf{3 . 3 4}$ & 5.93 & 9.2 & 8.96 & 15.6 \\
\hline
\end{tabular}



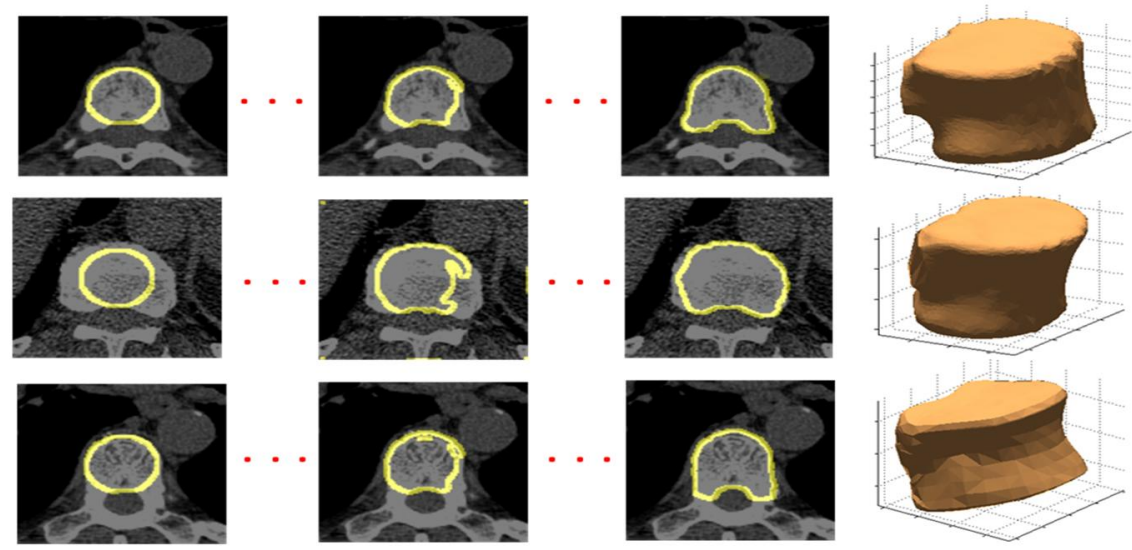

(b)

(c)

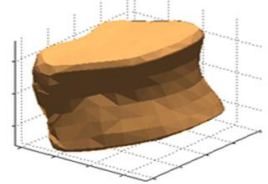

(a)

Figure 5.21. 2D Axial projections of various stages of level set evolution for three different vertebrae using proposed method A1, (a) initial disk, (b) intermediate stage, (c) the final stage, and (d) the resultant 3D vertebral body.
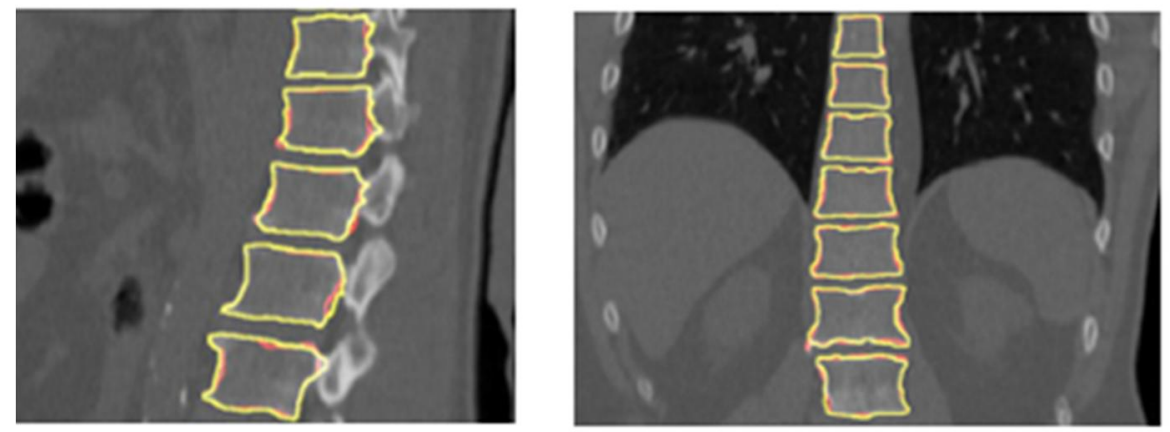

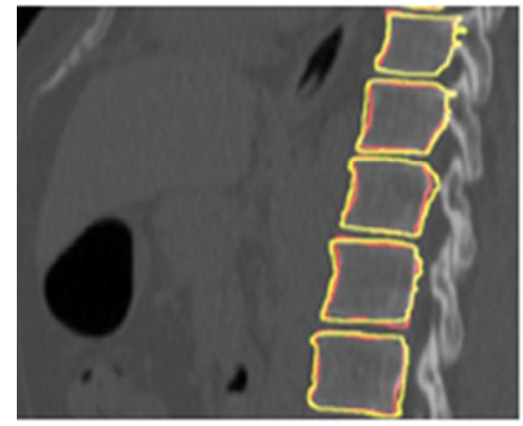

(a)

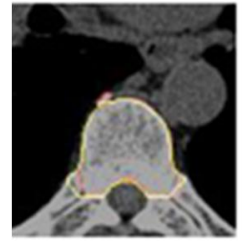

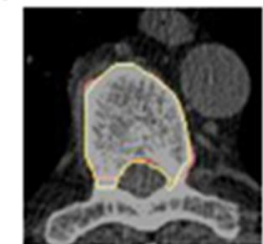

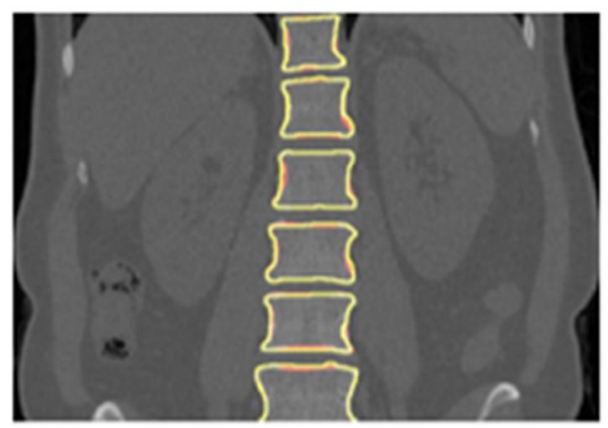

(b)
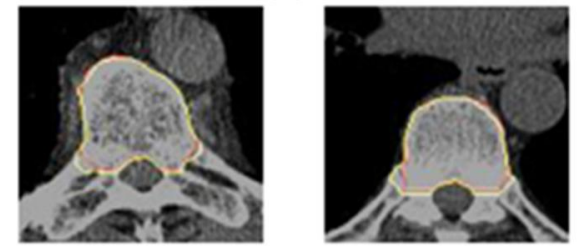

(c)

Figure 5.22. 2D projections of the 3D Segmentation method in (a) Sagittal, (b) Coronal, and (c) Axial views for different examples from the clinical data sets. (The red and yellow colors show the contour of the ground truths and segmented regions, respectively). 

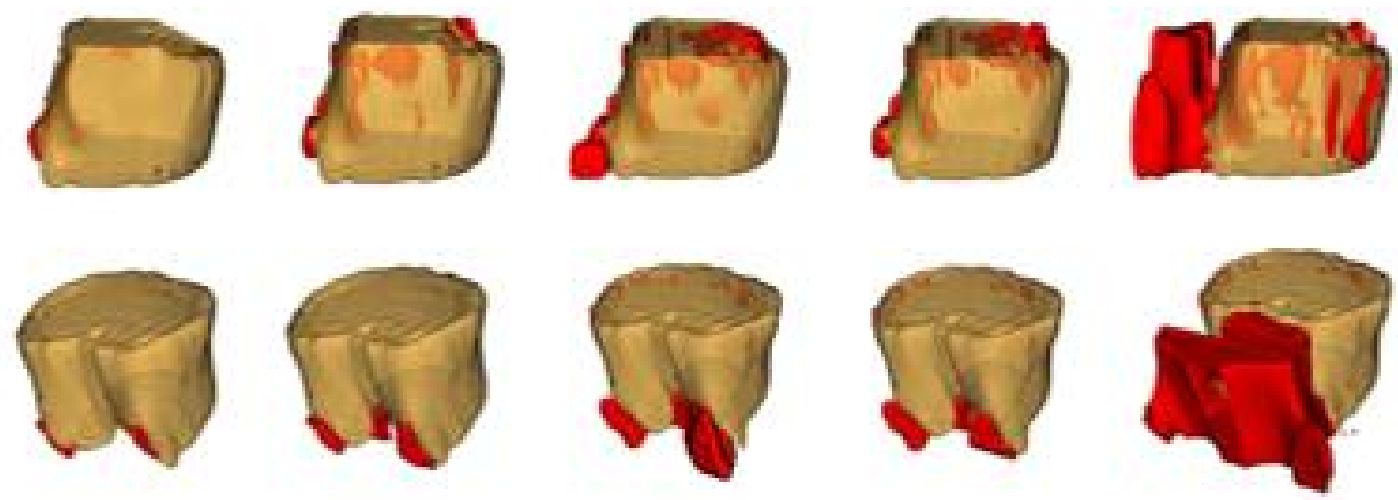

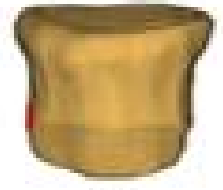

(a)

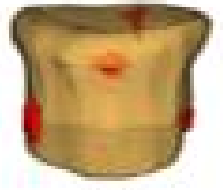

(b)

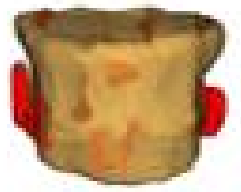

(c)

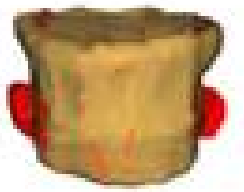

(d)

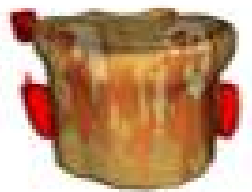

(e)

Figure 5.23. An example for the 3D segmentation of clinical data sets overlaid with ground truth (yellow) using different methods: (a) Proposed, A1, (b) Graph cuts with shape prior, A2, (c) AAM, A3, (d) Chen-Vese with shape prior, A4, and (e) The results of level sets, A5. The red color represents the misclassified voxels.

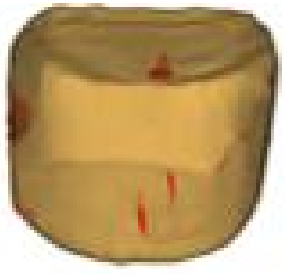

(a)

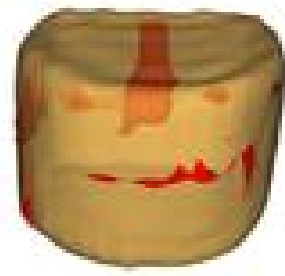

(b)

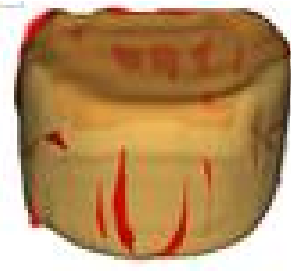

(c)

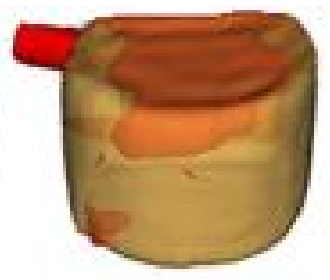

(b)

Figure 5.24. 3D segmentation results of ESP using the four different methods: (a) the result proposed method A1, (b) the result of Graph cuts with shape prior A2, (c) the result of Chen-Vese with shape prior A4, and (d) the result of level set method A5. The red color represents the misclassified voxels. 


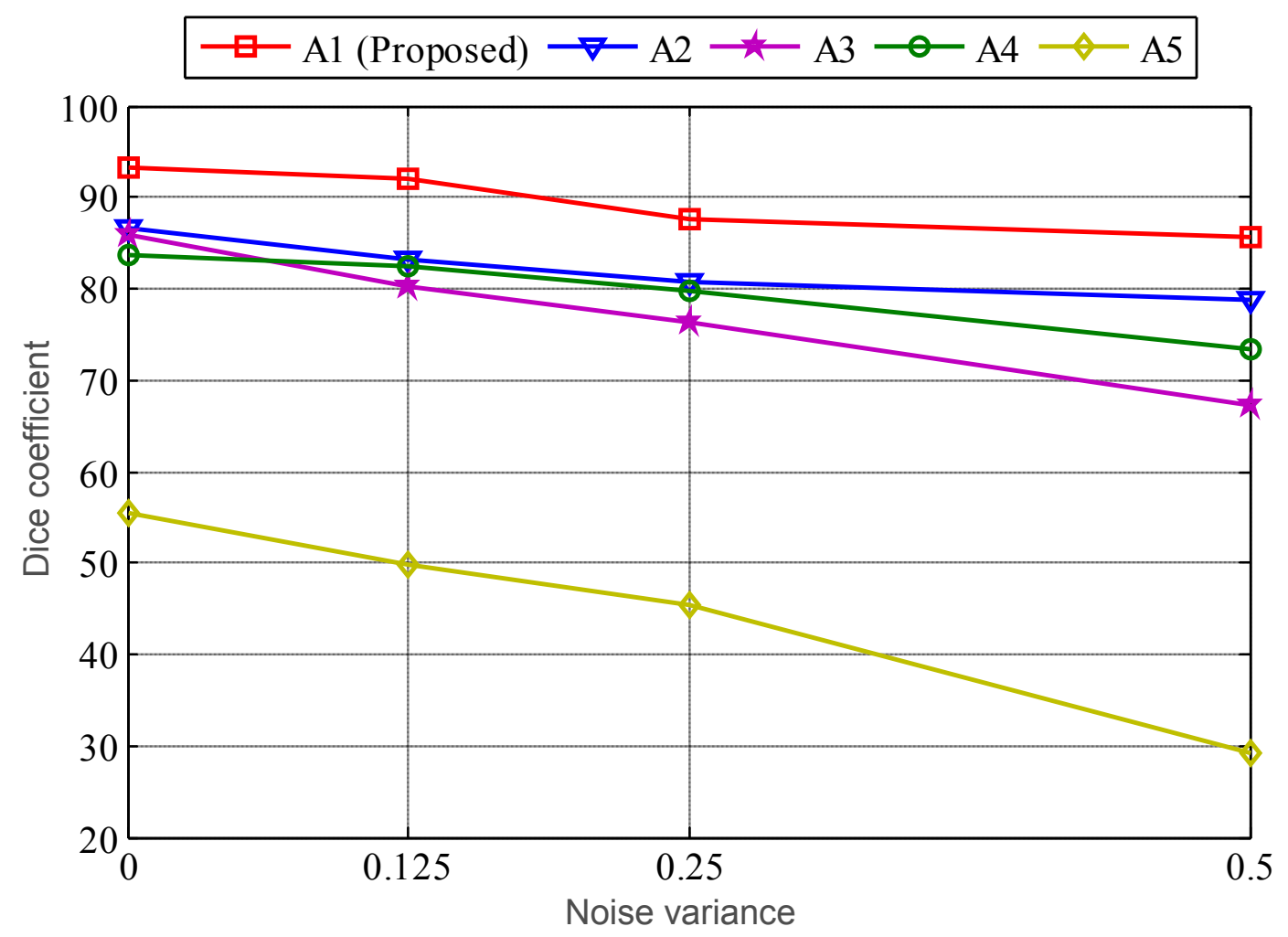

Figure5.25. The average segmentation accuracy (DC) of different segmentation methods on ESP dataset under various noise level. The results show that the proposed method is robust under various noise levels.

\subsection{Bone Mineral Density Measurements}

After segmentation, the ultimate goal of this work is to successfully obtain the BMD measurements with high trueness and precision in volumetric $\mathrm{CT}$ datasets. This is a crucial step for doctors and clinical experts who need to diagnose the low bone mass in a human body. In the experiments, we use the 53 volumetric VBs from thoracic and lumbar spine. Since spinal BMD measurements are limited to the VBs, the spine processes, which are successfully removed using the 3D shape prior, should not be included. We obtain the BMD measurements for each tested method to be compared with reference BMD measurement. Figure 5.26 represents the box-plot of the relative errors in BMD 


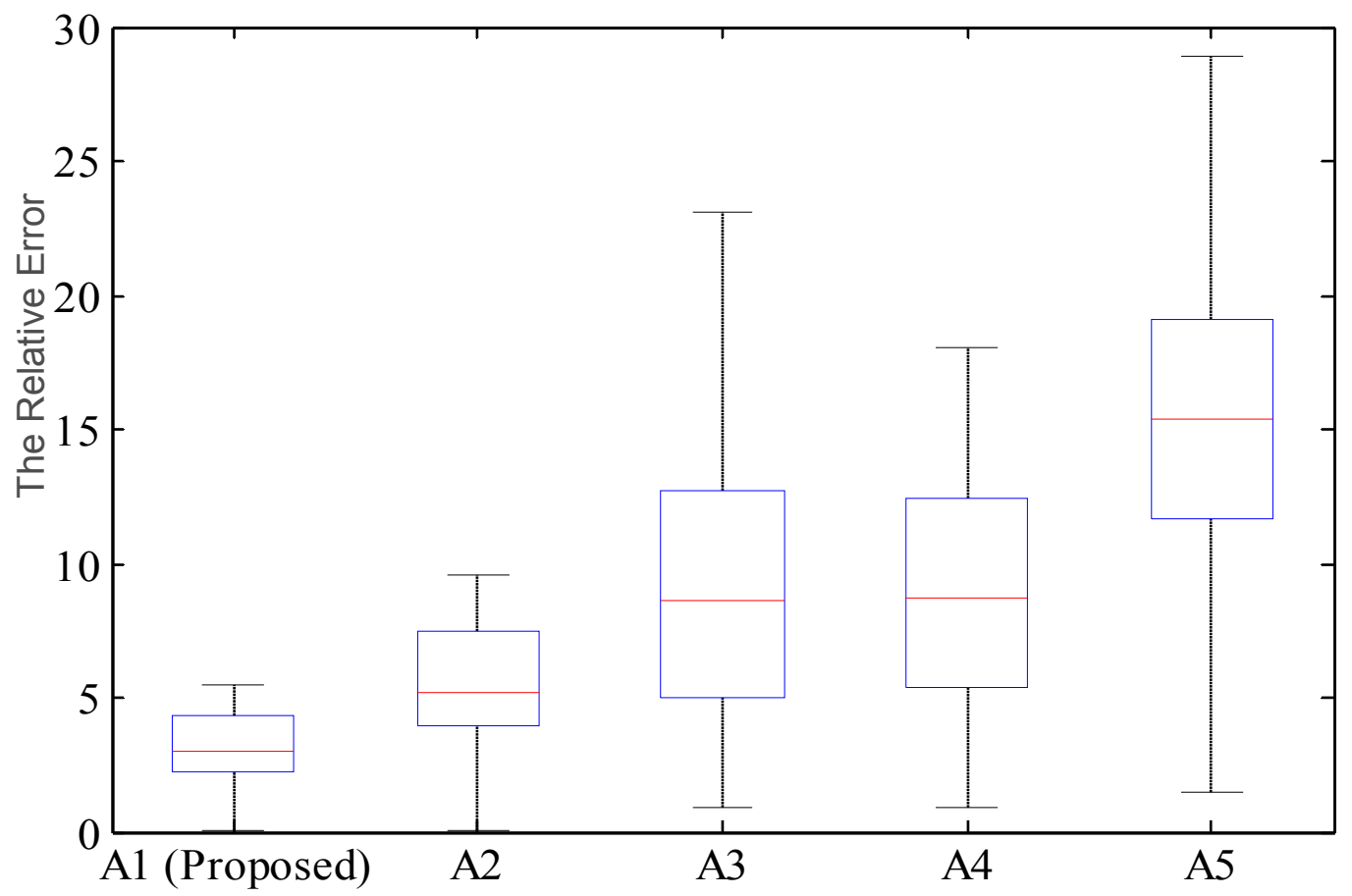

Figure5.26. Relative errors of BMD measurement of each method. The BMD measurement of the method (A1) has the lowest error and standard deviation.

measurements for each method (A1-A5) over the 53 VBs. Table 5.2 shows the average BMD relative error is $3.34 \%$ for the proposed method, which reflects how accurate the proposed segmentation approach is.

\subsection{Summary}

In this chapter, two new approaches for shape based segmentation of VBs were proposed in clinical CT images. The first approach is used foe 2D segmentation case. It depends on 2D-PCA for shape modeling and conventional level sets methods for segmentation step. The experimental results show that the noise immunity and the segmentation accuracy of 2D-PCA based approach are much higher than conventional 
PCA approach. Validity was analyzed using ground truths of clinical datasets as well as the European Spine Phantom (ESP).

On the other hand, a 3D simultaneous segmentation and registration approach was presented. This second approach incorporates both shape information and Willmore flow into the level set segmentation. To get the optimal segmentation, a new energy function using the appearance models and shape constraints was formulated and iteratively minimized using gradient descent. Experimental results confirm the degree of accuracy and robustness of the proposed framework. It has achieved $92.12 \%$ overall DC which is much higher than existing methods. Moreover, from the application point of view, the proposed shape based segmentation approach is helpful to eliminate the spinal processes which are not required for BMD analysis and FA. This leads to more accurate BMD measurements. 


\section{CHAPTER 6}

\section{D-3D REGISTRATION OF HUMAN ANKLE USING X-RAY AND CT IMAGES}

The registration of pre-operative $3 \mathrm{D}$ volumetric images to intra-operative $2 \mathrm{D}$ images provides an important way for relating the patient position and medical instrument location. In applications from orthopedics [67] to neurosurgery [66], it has a great value in maintaining up-to-date information about changes due to surgical intervention. The widely used 3D image modalities such as Magnetic Resonance Imaging (MRI), Computed Tomography (CT) and Positron Emission Tomography (PET) contain high resolution information about the imaged part of the human body. All these modalities can be greatly used for pre-operative procedure planning or evaluating an intervention post-operatively. However, the main drawback of these images is not completely reflecting the surgical situation, since they are static. In some applications it is important to use intra-operative images to follow the changes caused by the procedure or to visualize the location of a tool. In the operating room (OR), 2D images are more suitable for recording details about the current state. X-ray images are good examples of image modalities used for this purpose. Unfortunately, 2D images lack significant information that is present in the 3D modalities. So that, in order to relate between the OR 2D images and the detailed 3D model, experts need to mentally combine the information from the pre-operative and intra-operative images which is a very tough task. Therefore, it is useful to find a way to automate that procedure and making it reliable. The fusion of pre-operative and intra-operative images 


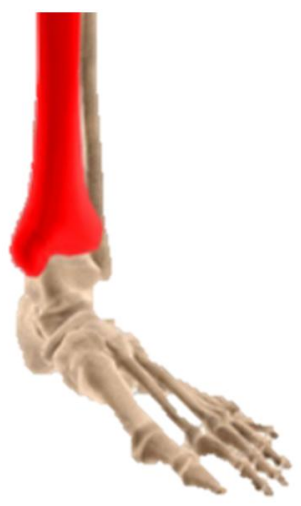

Tibia

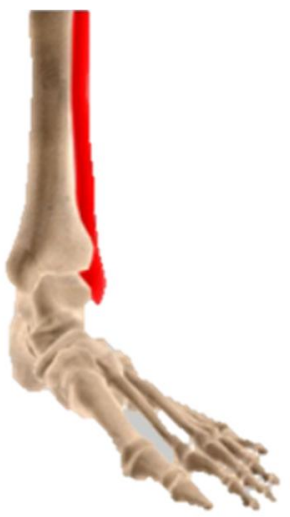

Fibula

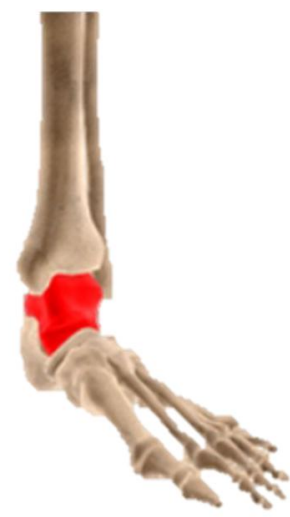

Talus

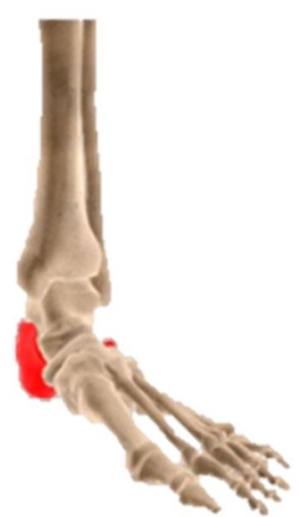

Calcaneus

Figure 6.1. Anatomy of the human foot.

will be meaningful if the components are properly aligned in space. To achieve this it is necessary to determine their relative position and orientation. The procedure that identifies a geometrical transformation that aligns two datasets is called registration. There are several approaches that can perform this task. Unfortunately, all of these techniques work on images of the same dimensionality, i.e. inputs are either 2D or 3D. But in this case, we need to align images with different dimensionality and combine the information from highresolution pre-operative datasets with the updated intra-procedural images. Additionally, as the registration results are expected during the medical procedure, the computation time would also be constrained.

In this chapter, a simple framework for 2D-3D registration of human ankle using X-Ray and CT Images is introduced. Our system consists of three main steps: 1) Projection of the pre-operative $3 \mathrm{D}$ volume to generate a synthetic $2 \mathrm{D}$ image, 2) Similarity measurement to quantify the quality of the alignment between the generated image and the reference (intra-operative) image, and 3) Optimization process to modify and refine current 
estimates of the problem parameters in a way that the similarity score is maximized. The rest of this chapter is organized as follows: Section 6.1 is related to the anatomy of the human foot and ankle bones. Section 6.2 talks about the long-term application of our work. Section 6.3 discusses the background of methods used in the experiment. Section 6.4 explains the experiments, and evaluates the accuracy of the results. Finally, summary is drawn in Section 6.5

\subsection{Anatomy of the Foot and Ankle}

The foot and ankle in the human body work together to provide balance, stability, movement, and Propulsion. This complex anatomy consists of: 26 bones, 33 joints, muscles, tendons, ligaments, blood vessels, nerves, and soft tissue. In order to understand conditions that affect the foot and ankle, it is important to understand the normal anatomy of the foot and ankle.

As shown in Figure 6.1, the ankle consists of three bones attached by muscles, tendons, and ligaments that connect the foot to the leg. In the lower leg are two bones called the tibia (shin bone) and the fibula. These bones articulate to the talus or ankle bone at the tibiotalar joint (ankle joint) allowing the foot to move up and down. The bottom of the talus sits on the heelbone, called the calcaneus.

\subsection{Ankle Fusion Surgery}

The majority of the medical applications for the proposed kind of registration have emerged in the field of radiology. Alignment information is important in planning, guidance and treatment procedures. This kind of work is crucial for the field of orthopedics and 


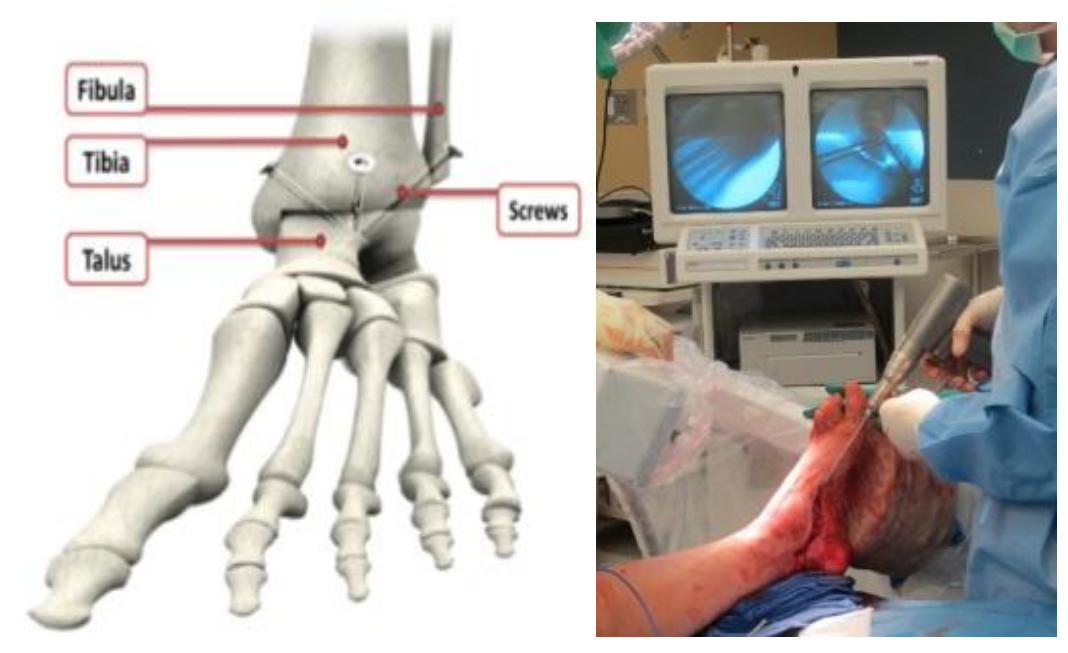

Figure 6.2. Ankle fusion surgery.

neuroradiology. It can be used in the following areas: Cranio-Catheter procedures, Metastatic Bone Cancer, Hip Replacement, and Spine Procedures [66].

The collaborators ${ }^{3}$ of this study are interested in applying the $2 \mathrm{D}-3 \mathrm{D}$ registration in the field of orthopedics. The major project is image-guided ankle fusion surgery. The longterm goal of this work is to apply this technique to ankle fusion surgery to determine the proper size and orientation of the screws which are used for fusing the bones together. In addition, we try to localize the best bone region to fix these screws. An ankle fusion is a surgical operation usually done when an ankle joint becomes worn out and painful. The most common cause of this pain is an ankle fracture. After a serious fracture, the joint may wear out and become painful. For example, a joint that is out of balance after it heals from a fracture can wear out faster than normal. As shown in Figure 6.2, an ankle fusion removes the surfaces of the ankle joint and allows the tibia to grow together, or fuse, with the talus

\footnotetext{
${ }^{3}$ R. Todd Hockenbury, M.D. Assistant Clinical Professor of Orthopaedic Surgery, University of Louisville
} 
[79]. The cut ends of the tibia and talus are brought together and held in place with three screws. Based on the intra-operative X-ray images, the doctor decides the size, the length, and the orientation of these screws. The ultimate goal is to enhance the quality of the surgical procedure in terms of time and accuracy, and would greatly reduce the need for repeated surgeries; thus, saving the patient's time, expense, and trauma.

\subsection{Proposed Method}

In this application, we focus on fusing $\mathrm{CT}$ and X-ray images. One of the key challenges when studying the $2 \mathrm{D}-3 \mathrm{D}$ registration problem is the need for an appropriate way to compare input datasets that are of different dimensionalities. One of the most common approaches is to simulate one of the modalities given the other dataset and an estimate about their relative spatial relationship, so that the images can be compared in the same space. Then a transformation $\mathrm{T}$ estimate can be updated to maximize the alignment according to some similarity measure. Most existing applications simulate 2D images from the 3D volume. It is more feasible to follow this approach. Simulated projection images that are to model the production of X-ray acquisitions from 3D volumetric CT are called Digitally Reconstructed Radiographs (DRRs). Figure 6.3 describes the main components of this framework. First, 3D translations $\left\{t_{x}, t_{y}, t_{z}\right\}$ and rotations $\left\{\theta_{x}, \theta_{y}, \theta_{z}\right\}$ to the CT volume are applied. Using the CT volume, we perform projection to generate the DRR. The projected image (DRR) is defined as the floating image and the X-ray image as the reference image. After the projection step, we have to identify a similarity measure that can quantify the quality of the alignment between the images and defining a procedure to 


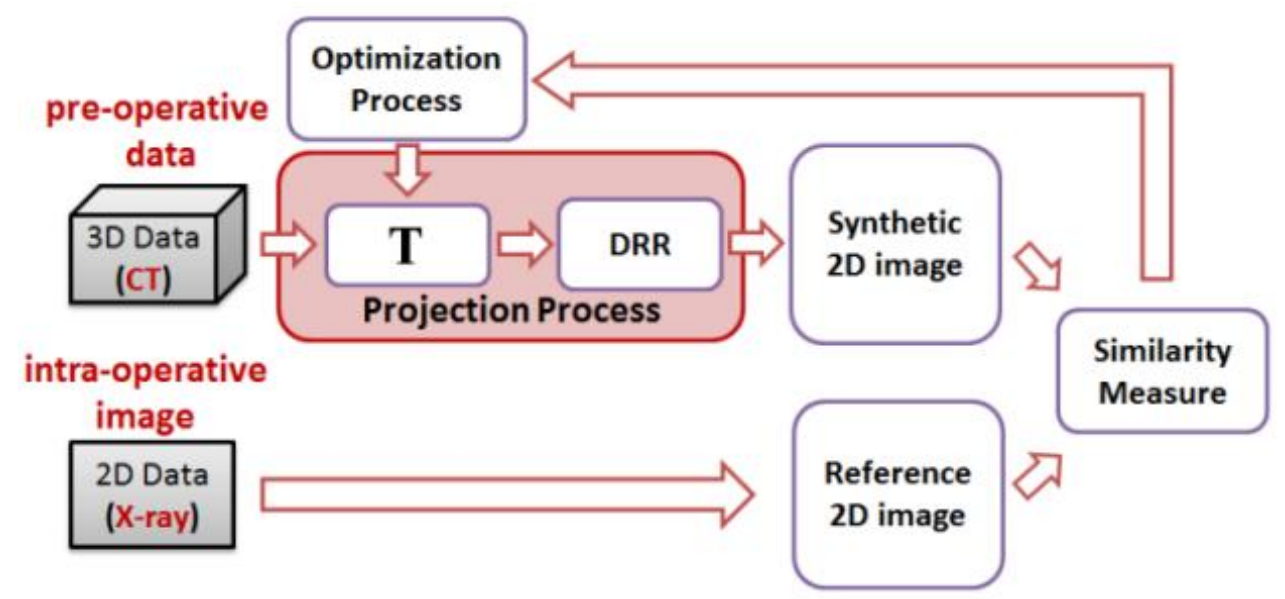

Figure 6.3. The block diagram of $2 \mathrm{D}-3 \mathrm{D}$ registration process.

modify and refine current estimates of the transformation parameters (rotation and translation) in a way that the similarity score is optimized. In other words, provided that a suitable similarity function is obtained, the best alignment parameters can be located with the help of an optimization procedure. More details about this framework will be discussed in the following subsections.

\subsubsection{Projection process}

A shear-warp factorization (SWF) method is used to generate synthetic 2D images from a given 3D CT volume (DRR images). It is one of the latest techniques of volume rendering [66]. In this method, a viewing transformation is applied to simplify the projection processing which is the mapping of world coordinates of the object into a virtual camera coordinates. The algorithm uses a principal viewing axis to choose a set of CT voxel slices to be resampled and composited. It also determines the order of the slices along the frontto-back direction of the image volume [71]. Let $M_{\text {view }}$ be a $4 \times 4$ affine viewing transformation matrix that transforms points from the object space to the image space.As 
in [71], $\mathrm{M}_{\text {view }}$ can be factorized as $\mathrm{M}_{\text {view }}=\mathrm{M}_{\text {warp }} \cdot \mathrm{M}_{\text {shear. }}$. As shown in Figure 6.4(a), in image space, let the viewing direction vector is $\overrightarrow{\boldsymbol{v}_{\boldsymbol{\imath}}}=(0,0,1)^{T}$ and $\overrightarrow{\boldsymbol{v}_{\mathbf{0}}}$ be the viewing direction vector transformed to object space. The relation between $\overrightarrow{\boldsymbol{v}_{\boldsymbol{\imath}}}$ and $\overrightarrow{\boldsymbol{v}_{\mathbf{0}}}$ will be:

$$
\overrightarrow{v_{\imath}}=M_{v i e w, 3 x 3} \cdot \overrightarrow{v_{0}}
$$

This system can be analytically solved for $\overrightarrow{\boldsymbol{v}_{\mathbf{0}}}$, yielding [72]:

$$
\overrightarrow{v_{0}}=\left[\begin{array}{lll}
m_{12} m_{2}-m_{2} & m_{13} \\
m_{2} m_{13}-m_{11} m_{2} \\
m_{11} m_{22}-m_{2} & m_{12}
\end{array}\right]
$$

where $m_{i, j}$ are elements of $M_{\text {view }}$. To get $\mathrm{M}_{\text {shear, }}$, assume that the principal viewing axis is the $+\mathrm{Z}$ axis of the object coordinate system (see Figure6.4). This shear causes the viewing direction to become perpendicular to the slices of the volume. To do that, the volume should be sheared in the $\mathrm{x}$ - direction by: $-v_{o, x} / v_{o, z}$. A similar argument holds for the shear in the $\mathrm{Y}$ direction. Thus the shear coefficients will be:

$$
\begin{aligned}
& S h_{x}=-\frac{v_{0, x}}{v_{o, z}}=\frac{m_{22} m_{13}-m_{12} m_{23}}{m_{11} m_{22}-m_{2} m_{12}} \\
& S h_{y}=-\frac{v_{0, y}}{v_{o, z}}=\frac{m_{11} m_{23}-m_{2} m_{13}}{m_{11} m_{22}-m_{2} m_{12}}
\end{aligned}
$$

Thus, the shear transformation matrix $\mathrm{M}_{\text {shear }}$ can be described as:

$$
\mathbf{M}_{\text {Shear }}=\left[\begin{array}{cccc}
1 & 0 & S h_{x} & 0 \\
0 & 1 & S h_{y} & 0 \\
0 & 0 & 1 & 0 \\
0 & 0 & 0 & 1
\end{array}\right]
$$

The second factor of the viewing matrix describes how to warp the intermediate image into the final image. So, we can get: 


$$
M_{\text {warp }}=M_{\text {view }} \cdot \mathbf{M}_{\text {Shea } r}^{-1}=M_{\text {view }} \cdot\left[\begin{array}{cccc}
1 & 0 & -S h_{x} & 0 \\
0 & 1 & -S h_{y} & 0 \\
0 & 0 & 1 & 0 \\
0 & 0 & 0 & 1
\end{array}\right] \text {, }
$$

Figure 6.4(b) shows samples for projected 2D images using SWF approach with different viewing parameters (i.e. different $t_{x}, t_{y} t_{z}, \theta_{x}, \theta_{y}, \theta_{z}$ ). The average elapsed time required to generate a DRR image (based on SWF) is $3.92 \pm 0.45$ seconds $^{4}$. More details about SWF and volume rendering techniques can be found in [66]-[72].

\subsubsection{Similarity Measure}

In many registration systems, the quality of alignment is scored by objective functions. Common registration methods can be grouped into two major categories based upon the nature of the similarity measure to which they apply: they can be classified as feature or intensity-based [72].

Feature-based methods rely on the identification of natural landmarks in the input images in order to determine the best alignment. It is necessary to segment the most significant features in both of the input images and the matching criterion is then optimized with respect to them.

Intensity-based methods operate on the pixel intensities directly. They calculate various statistics using the intensity values of the inputs which are then compared in the images to be aligned.

According to literatures, intensity-based similarity measures are more suitable for 2D3D applications [68]. They suggested many objective functions that can be used in

\footnotetext{
${ }^{4}$ As compared with conventional volume rendering methods (e.g. ray-casting), the SWF is significantly fast and robust.
} 
matching X-ray and CT images. For example: normalized cross-correlation [68], pattern intensity [70], normalized mutual information (NMI) [73], [74], gradient correlation [72] and gradient difference [68].

In the proposed framework, two novel image similarity measures are adopted from [77] and [80]. The first one is called exponential correlation (EC). The other is called pixelbased individual entropy correlation coefficient (IECC). Both are used as the similarity measure between the DRR images and the reference X-ray image in order to evaluate the current quality of alignment.

\subsubsection{Exponential Correlation (EC)}

Given that the real X-ray image is the reference image $(\mathrm{R})$ and the DRR image is the floating image (F), their EC value can be calculated using the following equation [81]:

$$
\operatorname{EC}(R, F)=E\left[\left(e^{\mathbf{R}(\mathbf{x})-\overline{\mathbf{R}}}-\mathbf{1}\right)\left(e^{\mathrm{F}(\mathbf{x})-\overline{\mathrm{F}}}-\mathbf{1}\right)\right]
$$

where $\mathbf{x}$ stands for the coordinates vector of the image, the vector $\mathbf{x}$ is defined on the set $D_{\mathrm{x}}$ defined as $\mathbf{F} \cap \mathbf{R}$, and $\boldsymbol{E}[$.$] denotes the expectation operator over the D_{\mathrm{x}} . \overline{\mathbf{F}}$ and $\overline{\mathbf{R}}$ represent the mean of intensity values of images $\mathrm{F}$ and $\mathrm{R}$ respectively. When two images are geometrically aligned, EC value is maximized [81].

\subsubsection{Individual Entropy Correlation Coefficient (IECC)}

In these types of similarity measures, we deal with the images $\mathrm{R}$ and $\mathrm{F}$ as two random variables. A $1 \mathrm{D}$ histogram is constructed for each image. It shows the distribution of the pixel values. Since these values vary over a wide range, they were rescaled into $N=64$ bins. A 2D histogram $h(r, f)$ is obtained from the pair of floating image and reference image. Each entry in this histogram represents the number of times intensity $r$ in image $\mathrm{R}$ coincides 
with intensity $f$ in the other image $\mathrm{F}$. The probability distribution of this $2 \mathrm{D}$ histogram values is obtained from $h(r, f)$. It is called the joint probability distribution and can be expressed as [80]:

$$
p\left(r_{i}, f_{j}\right)=\frac{h\left(r_{i}, f_{j}\right)}{\sum_{i=1}^{N} \sum_{j=1}^{N} h\left(r_{i}, f_{j}\right)} .
$$

As discussed in chapter 4 , the pixel-based IECC depends on $p\left(r_{i}, f_{j}\right)$. It represents the ratio between the pixel-based component of the mutual information between the two images, and the sum of the pixel-based components of the two marginal entropies of each image. So, IECC is expressed as [82]:

$$
\operatorname{IECC}(R, F)=\sum_{i=1}^{N} \sum_{j=1}^{N} \frac{p\left(r_{i}, f_{j}\right) \log _{2}\left(\frac{p\left(r_{i}, f_{j}\right)}{p\left(r_{i}\right) p\left(f_{j}\right)}\right)}{p\left(r_{i}\right) \log _{2}\left(r_{i}\right)+p\left(f_{j}\right) \log _{2}\left(f_{j}\right)},
$$

where $p\left(r_{i}\right)$ and $p\left(f_{j}\right)$ are the marginal probability distribution of each image. When two images are geometrically aligned, IECC value is maximized. For more details, see [82].

\subsubsection{Optimization process}

Provided that a suitable similarity function is obtained, the best alignment parameters can be estimated with the help of an optimization process. The optimization process aims to maximize the similarity score between images. There are two major classes of optimization approaches: non-gradient and gradient methods. The first class might be easier to implement as it requires only the evaluation of the objective function and no additional computations to derive the consecutive search directions. However, the second could potentially be much faster as its search is guided towards the maximum. For simplicity, Nelder-Mead method (one of non-gradient methods) is used in the system [67]. 


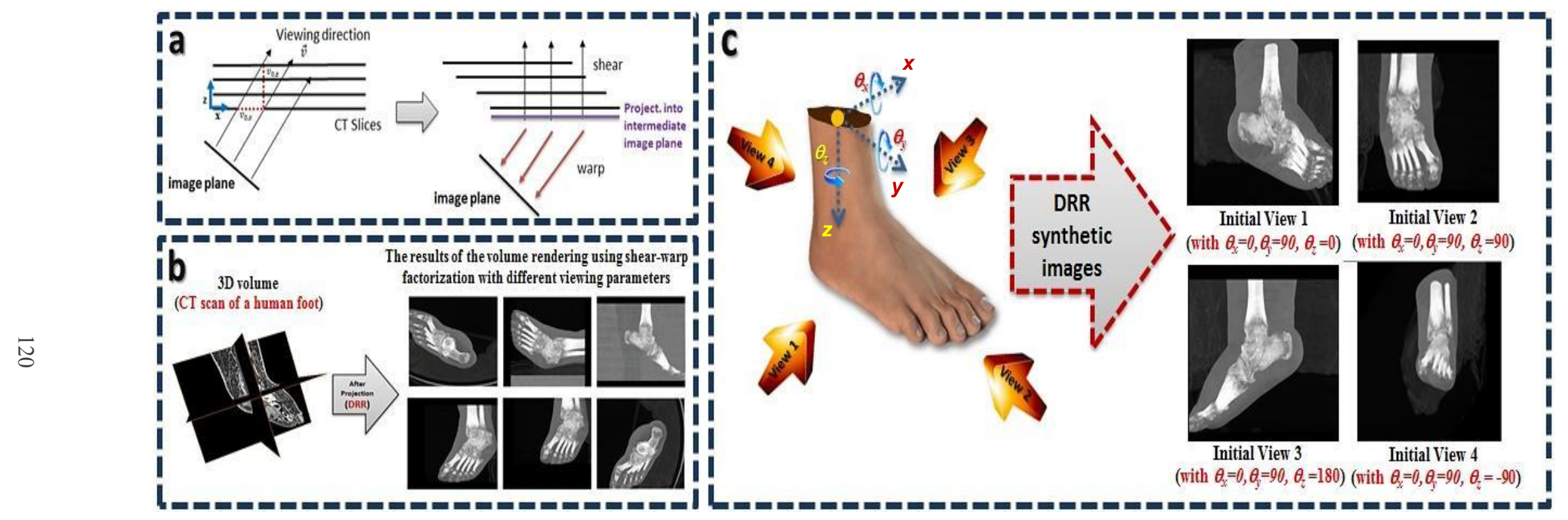

Figure 6.4. (a) Shear-warp factorization idea. (b) Examples for DRR based on SWF. (c) The initialization scenarios. 


\subsection{Experimental Results}

In this chapter, the framework is applied on clinical CT ankle datasets. The goal is to register these pre-operative $\mathrm{CT}$ data to intra-operative $2 \mathrm{D}$ images. The clinical datasets were scanned at $120 \mathrm{KV}$ with $2.5 \mathrm{~mm}, 1.33 \mathrm{~mm}$, or $0.42 \mathrm{~mm}$ slice thicknesses. Our algorithm has been tested on $1500 \mathrm{CT}$ slices which are obtained from 22 different patients. Regarding the intra-operative $2 \mathrm{D}$ images, the ray-casting volume rendering technique (excerpted from [68]) to generate ground truth X-ray images with different viewing transformations (i.e. known $t_{x}, t_{y}, t_{z}, \theta_{x}, \theta_{y}, \theta_{z}$ ) that represent the intra-operative $\mathrm{X}$-ray images is used. As a pre-processing step, the brightness of these X-ray images are adjusted to roughly segment the soft tissues from the ankle bones (see Figure6.5a). These images are used to test the quality of the system. For the initialization, we exploit the setup of the intraoperative imaging machine to choose the proper initial orientation as shown in Figure6.4c (i.e. according to the intra-operative image, we initially start the DRR from view 1,2, 3 or 4).

A variety of methods to measure the accuracy of this framework is adopted. First, the checkerboard representation is used to visually evaluate the registration quality of the framework (see Figure $6.5 \mathrm{~d}$ and $6.5 \mathrm{e}$ ). In these examples, IECC is calculated as a similarity measure. Having a closer look at Figure $6.5 \mathrm{e}$, it shows that the registration is very accurate, since the two parts coming from different images have no transition. . Edges of the foot bone are connected along the squares in all parts of the checkerboard image. Second, the correlation coefficient (defined in (3.3)) between the image $\mathrm{R}$ and the $\mathrm{F}$ is computed before and after the registration process using NMI (See Chapter 4), EC, and IECC for different 
examples with different viewing parameters. Additionally, the execution time required for each example is measured (in minutes). The obtained results are summarized in Table 6.1. These results indicate that: For the interventional applications, although EC based framework is not the most accurate, it has the advantage of reducing the execution time by almost two third as compared to NMI and IECC. On the other hand, if the accuracy is sought, IECC outperforms the other two systems with comparative execution time to NMI approach. Finally, the mean error -and its standard deviation SD- of the estimated registration parameters $\left\{t_{x}, t_{y}, t_{z}, \theta_{x}, \theta_{y}, \theta_{z}\right\}$ using NMI, EC and IECC are summarized in Table 6.2. This error is the average absolute difference between the ground truth parameters and the final estimated parameters after registration of a given view for all CT datasets. Again, and according to these results, the accuracy of the IECC framework is higher than the systems that are based on the other similarity measures; NMI and EC.

\subsection{Summary}

In this chapter, a new framework for registering pre-operative 3D volumetric data to intraoperative 2D images was introduced into the field of orthopedics, specifically on ankle surgery. The framework was implemented based on SWF rendering techniques with Exponential Correlation (EC) or Individual Entropy Correlation Coefficient (IECC) as new similarity measures for the $2 \mathrm{D}-3 \mathrm{D}$ registration process. It was tested on different clinical CT scans of human ankle and foot. Experiments demonstrated that the EC-based framework is fast and performs almost as much as NMI which is compatible with the time limitation of the interventional applications. From the accuracy point of view, the IECC- 

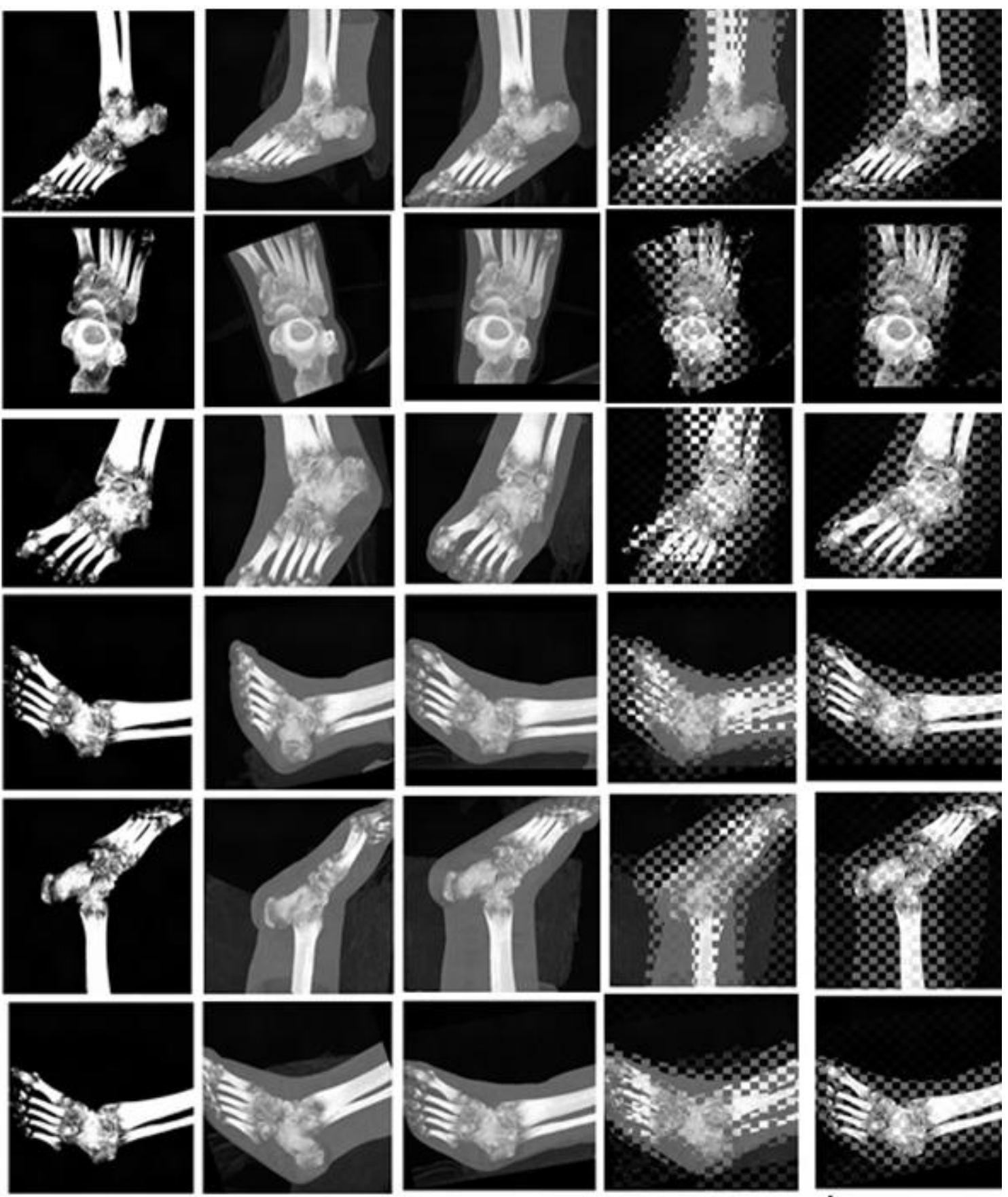

(a)

(b)

(c)

(d)

(e)

Figure 6.5. 2D-3D Registration results for different examples with different views for one of the clinical datasets using IECC as a similarity measure: (a) is the reference image (represents the intraoperative X-ray generated by ray-casting algorithm) (b) is the initial floating (synthetic) image, generated by SWF algorithm (c) is the final floating image after registration, (d) is checkerboard representation before registration and (e) after registration. 
based framework is the most accurate system with comparative execution time to NMIbased system.

Table 6.1. Correlation coefficient of the 2D-3D registration framework using NMI, EC and IECC for different views. The red values represent the execution time (in minutes).

\begin{tabular}{ccccc}
\hline \multirow{2}{*}{ Example } & \multicolumn{5}{c}{ Correlation Coefficient/ Execution Time (In Minutes) } \\
\cline { 2 - 5 } & $\begin{array}{c}\text { Before } \\
\text { Registration }\end{array}$ & $\begin{array}{c}\text { After Registration } \\
\text { (NMI) }\end{array}$ & $\begin{array}{c}\text { After Registration } \\
(\text { EC) }\end{array}$ & $\begin{array}{c}\text { After Registration } \\
\text { (IECC) }\end{array}$ \\
\hline View 1 & 0.6297 & $0.8234 / 2.9$ & $0.8174 / 1.1$ & $0.9974 / 2.7$ \\
\hline View 2 & 0.6102 & $0.8511 / 2.7$ & $0.8741 / 0.9$ & $0.9851 / 2.5$ \\
\hline View 3 & 0.5162 & $0.8923 / 3.0$ & $0.8886 / 1.2$ & $0.9904 / 3.1$ \\
\hline View 4 & 0.5716 & $0.8959 / 3.2$ & $0.8896 / 0.9$ & $0.9934 / 2.9$ \\
\hline View 5 & 0.5234 & $0.8738 / 3.6$ & $0.8835 / 1.3$ & $0.9884 / 3.2$ \\
\hline View 6 & 0.6453 & $0.9003 / 2.3$ & $0.8921 / 0.7$ & $0.9986 / 2.0$ \\
\hline View 7 & 0.5015 & $0.812 / 3.3$ & $0.8236 / 1.5$ & $0.9158 / 3.2$ \\
\hline View 8 & 0.4325 & $0.7887 / 4.2$ & $0.7912 / 2.1$ & $0.8368 / 4.0$ \\
\hline View 9 & 0.6235 & $0.8325 / 2.8$ & $0.8553 / 0.9$ & $0.9684 / 2.6$ \\
\hline View 10 & 0.5108 & $0.8351 / 3.2$ & $0.8125 / 1.3$ & $0.9213 / 2.9$ \\
\hline
\end{tabular}

Table 6.2. Mean registration error and SD of the estimated parameters.

\begin{tabular}{|c|c|c|c|c|c|c|c|}
\hline \multicolumn{2}{|c|}{ Parameters } & $t_{x}(\mathrm{~mm})$ & $t_{y}(\mathrm{~mm})$ & $t_{z}(\mathrm{~mm})$ & $\theta_{x}($ deg $)$ & $\theta_{y}($ deg $)$ & $\theta_{z}($ deg $)$ \\
\hline \multirow{2}{*}{$\underset{0}{0}$} & NMI & $1.1 \pm 0.91$ & $1.2 \pm 0.89$ & $1.4 \pm 0.98$ & $1.0 \pm 0.77$ & $1.2 \pm 0.87$ & $0.6 \pm 0.62$ \\
\cline { 2 - 8 } & EC & $1.3 \pm 0.86$ & $0.9 \pm 0.79$ & $1.6 \pm 0.99$ & $0.9 \pm 0.78$ & $1.3 \pm 1.01$ & $0.7 \pm 0.48$ \\
\cline { 2 - 8 } & IECC & $0.9 \pm 0.52$ & $0.8 \pm 0.41$ & $1.1 \pm 0.69$ & $0.7 \pm 0.36$ & $0.9 \pm 0.41$ & $0.4 \pm 0.29$ \\
\hline
\end{tabular}




\section{CHAPTER 7}

\section{CONCLUSION AND FUTURE DIRECTIONS}

This dissertation has presented different shape/image registration techniques with two new applications to medical imaging field.

\subsection{Conclusion}

In this dissertation, two different shape based approaches to segment the human VBs were presented. These approaches are considered as a direct application for shape registration.

The first approach is used for 2D segmentation case. It depends on 2D-PCA for shape modeling and conventional level sets methods for segmentation step. The experimental results show that the noise immunity and the segmentation accuracy of 2DPCA based approach are much higher than conventional PCA approach. Validity was analyzed using ground truths of clinical datasets as well as the European Spine Phantom (ESP).

For the $3 \mathrm{D}$ case, a simultaneous segmentation and registration approach was presented. This second approach incorporates both shape information and Willmore flow into the level set segmentation. To get the optimal segmentation, a new energy function using the appearance models and shape constraints was formulated and iteratively minimized using gradient descent. Experimental results confirm the degree of accuracy and robustness of the proposed framework. It has achieved $92.12 \%$ overall DC which is much higher than existing methods. Moreover, from the application point of view, the proposed 
shape based segmentation approach is helpful to eliminate the spinal processes which are not required for BMD analysis and FA. This leads to more accurate BMD measurements.

On the other hand, a new framework for registering pre-operative 3D volumetric data to intra-operative 2D images was introduced into the field of orthopedics, specifically on ankle surgery. The framework was implemented based on shear-warp factorization (SWF) rendering techniques with Exponential Correlation (EC) or Individual Entropy Correlation Coefficient (IECC) as new similarity measures for the 2D-3D registration process. It was tested on different clinical CT scans of human ankle and foot. Experiments demonstrated that EC-based framework is fast and performs almost as much as NMI which is compatible with the time limitation of the interventional applications. From the accuracy point of view, the IECC-based framework is the most accurate system with comparative execution time to NMI-based system. The proposed approach can be considered as a step towards a robust image-guided surgical station for ankle fusion surgery.

\subsection{Future Directions}

Future works can be directed as follows:

- For the segmentation of the $V B$, although the proposed segmentation method in Chapter 5 is able to work on 2D/3D data sets, the shape registration is accomplished slice by slice. This work can be upgraded and tested in 3D framework. In this case, the user should expect an increased execution time since the registration parameters will be increased to 9 from 6 . 
- The high execution time of the shape registration via gradient descent approach can be decreased by adopting modern graphics processing units (GPUs).

- In the literature, the segmentation is coupled with the pose estimation such as Sandhu et al. proposed [83]. They present a non-rigid approach to jointly solving the tasks of 2D-3D pose estimation from a 2D scene and 2D image segmentation. The proposed work in Chapter 5 can be upgraded using the similar idea.

- For 2D-3D registration of the ankle fusion, Future directions are geared towards formulating a new objective function and implementing an advanced optimization technique to expand this work.

- We are also aiming to apply the 2D-3D registration on real X-ray image (Not synthetic images). This requires a dataset of X-ray and $\mathrm{CT}$ images for the same patient. Also, it is very important to validate the proposed framework on a large number of datasets (up to100 scans).

- Optimize the computational time of the registration process by adopting modern GPUs for direct volume rendering. 


\section{REFERENCES}

[1] X. Huang, N. Paragios, and D.N. Metaxas. Shape registration in implicit spaces using information theory and free form deformations. IEEE Transactions on Pattern Analysis and Machine Intelligence, 28(8):1303-1318, 2006.

[2] B. Hong, E. Prados, S. Soatto, and L. Vese. Shape representation based on integral kernels: Application to image matching and segmentation. In Proc. of IEEE CVPR'06, pages 833-840, Washington, DC, USA, 2006.

[3] H. AbdEl-Munim and A. A. Farag. Shape representation and registration using vector distance functions. In Proc. IEEE CVPR'07, Minneapolis, Minnesota, USA, 2007.

[4] B. Jian and B. Vemuri. Robust point set registration using gaussian mixture models. IEEE TPAMI, 33(8):1633-1645, 2011.

[5] S. Belongie, J. Malik, and J. Puzicha. Shape matching and object recognition using shape contexts. IEEE TPAMI, 24(24):509-522, 2002.

[6] T. B. Sebastian, P. N. Klein, and B. B. Kimia. Recognition of shapes by editing their shock graphs. IEEE TPAMI, 26(5):550-571, 2004.

[7] L. H. Staib and J. S. Duncan. Boundary finding with parametrically deformable models. IEEE TPAMI, 14(11):1061-1075, 1992.

[8] M. Holden. A review of geometric transformations for nonrigid body registration. Medical Imaging, IEEE Transactions on, 27(1):111-128, 2008.

[9] H. Chui and A. Rangarajan. A new algorithm for non-rigid point matching. In Proc. of IEEE CVPR'00, 2000.

[10] H. Chen and B. Bhanu. Global-to-local non-rigid shape registration. In Proc. of ICPR'06, Washington, DC, USA, 2006.

[11] A. J. Yezzi and S. Soatto. Deformotion: Deforming motion, shape average and the joint registration and approximation of structures in images. Int. J. Comput. Vision, 53(2), 2003.

[12] Y. Zheng and D. Doermann. Robust point matching for two-dimensional nonrigid shapes. In Proc. of IEEE ICCV’05, pages 1561-1566, 2005.

[13] X. Huang, N. Paragios, and D.N. Metaxas. Establishing local correspondences towards compact representations of anatomical structures. In Proc. of MICCAI'03, Montreal, 2003.

[14] H. Abd El Munim, Implicit Curve/Surface Evolution with Application to the Image Segmentation Problem. Ph.D Thesis, University of Louisville, May, 2007. 
[15] N.D. Salih, and D. C. L Ngo, An Efficient Boundary-Based Approach for Shape Representation. Information and Communication Technologies, 2006. ICTTA '06. 2nd, 2006.

[16] T. F. Cootes, and Taylor, C. J. and Cooper D. H. and Graham J. Active shape models - their training and application. Computer Vision and Image Understanding, pages 38-51, 1995.

[17] T. F Cootes,. and Edwards, G. J. and Taylor, C. J. Active Appearance Models. Proc. European Conference on Computer Vision, 1998.

[18] T. F. Cootes, and Edwards, G. J. and Taylor, C. J. Active Appearance Models. IEEE Transactions on Pattern Analysis and Machine Intelligence, 23(6):681-685, 2001.

[19] M. Kass, and A. Witkin, and Terzopoulos, D. Snakes: Active contour models. Int. J. of Computer Vision, 1(4):321-331, 1988.

[20] S. Osher, and J.A. Sethian, Fronts propogating with curvature-dependent speed: algorithms based on Hamilton-Jacobi formulations. J. Comp. Phys., 79:12-49, 1988.

[21] N. Paragios, and Rousson, M. and V. Ramesh, Matching Distance Functions: A Shape-to-Area Variational Approach for Global-to- Local Registration. Proc. Seventh European Conf. Computer Vision, pages 775-789, 2002.

[22] A. Gostasby, Image Registration Tutorial. Proc. of Computer Vision and Pattern Recognition, 2004.

[23] J. M. Fitzpatrick, and D. L. G. Hill, and Maurer, Jr. C. R. Image Registration. Chapter 8 of "Handbook of Medical Imaging,Milan Sonka and J. Michael Fitzpatrick, eds., SPIE Press, 2000.

[24] L. K. Nielsen, Elastic Registration of Medical MR Images. Cand. Scient. Thesis in Computational Science, University of Bergen, 2003.

[25] N. Paragios, M. Rousson, and Visvanathan Ramesh, "Non-rigid registration using distance functions," Computer Vision and Image Understanding vol. 8, 2003.

[26] H. AbdEl-Munim and A. A. Farag. A variational approach for shapes registration using vector maps. In Proc. of the 2006 IEEE International conference on Image Processing, Washington, DC, USA, October 8-11, 2006.

[27] H. Abd EL Munim, A. A. Farag and A. A. Farag, Shape Representation and Registration in Vector Implicit Spaces: Adopting a Closed-Form Solution in the Optimization Process, IEEE Transactions on Pattern Analysis and Machine Intelligence, Vol. 35, March 2013.

[28] R. Fahmi, A. Abdel-Hakim Aly, A. El-Baz, and A. Farag. New deformable registration technique using scale space and curve evolution theory and a finite element based validation framework. In 28th Annual Int. Conf. of the IEEE Engineering in Medicine and Biology Society, pages 3041-3044, New York, NY, Aug-Sep 2006. 
[29] R. Fahmi, Variational Methods For Shape and Image Registrations, PhD dissertation, Computer Vision and Image Processing Laboratory, University of Louisville, May, 2008.

[30] M. Aslan, A. Shalaby, and A.A. Farag, Clinically Desired Segmentation Method for Vertebral Bodies, Proc. of 2013 International Symposium on Biomedical Imaging (ISBI'13), San Francisco, USA, April 7-11, 2013.

[31] P. Elsen, E. Pol, and M. Viergever, Medical image matching a review with classification, Engineering in medicine and biology, vol. 12, Mar. 1993.

[32] C. Davatzikos, J. Prince, and R. Bryan, Image registration based on boundary mapping, IEEE Trans. on Medical Imaging, vol. 15, no. 1, Feb. 1996.

[33] D. Vandermeulen, A. Collignon, J. Michiels, H. Bosmans, P. Suetens, G. Marchal, G. Timmens, P. van den Elsen, M. Viergever, H. Ehricke, D. Hentschel, and R. Graumann, Multi-modality image registration within covira, Studies in health, technology and informatics, vol. 19, pp. 29-42, 1995.

[34] P. Viola and W. Wells, Alignment by maximization of mutual information, Int. Journal of Computer Vision, vol. 24, no. 2, pp. 137-154, 1997.

[35] J. Pluim, J. Maintz, and M. Viergever, Interpolation artefacts in mutual information based image registration, Computer Vision and Image Understanding, vol. 77, pp. 211-232, 2000.

[36] H. Neemuchwala and A. Hero, Entropic graphs for registration in Multi-sensor Image Fusion and its Applications, R. Blum and Z. Liu, Eds., 2004.

[37] F. Maes, A. Collignon, D. Vandermeulen, G. Marchal, and P. Seutens, Multimodality image registration by maximization of mutual information, IEEE Trans. on Medical Imaging, vol. 16, no. 2, pp. 187-198, 1997.

[38] F. Maes, D. Vandermeulen, and P. Suetens, Comparative evaluation of multiresolution optimization strategies for multimodality image registration by maximization of mutual information, Med. Image Anal., vol. 3, no. 4, pp. 373-386, 1999.

[39] J. V. Hajnal, D. L. Hill, and D. J. Hawkes, eds., Medical Image Registration, CRC Press, 2001.

[40] G. P. Penney, J. Weese, J. A. Little, P. Desmedt, D. L. Hill, And D. J. Hawkes, A Comparison of Similarity Measures for Use in 2D-3D Medical Image Registration, Lecture Notes in Computer Science, 2001.

[41] J. H. Hipwell, G. P. Penney, T. C. Cox, J. V. Byrne, and D. J. Hawkes, 2D-3D Intensity Based Registration of DSA and MRA - A Comparison of Similarity Measures, Lecture Notes in Computer Science, 2002.

[42] B. Fei, J. L. Duerk, D. T. Boll, J. S. Lewin, and D. L. Wilson, Slice-to-Volume Registration and its Potential Application to Interventional MRI-Guided Radio- 
Frequency Thermal Ablation of Prostate Cancer, IEEE Transactions on Medical Imaging, 2003.

[43] F. Wang, B. C. Vemuri, M. Rao, and Y. Chen, Cumulative Residual Entropy, A New Measure of Information \& its Application to Image Alignment, International Conference on Computer Vision, 2003.

[44] J.B. Antoine Maintz and Max A. Vierger, A Survey of Medical Image Registration ,Medical Image Analysis, vol 2(1),1998.

[45] C. Kuglin and D. Hines, The phase correlation image alignment method, Proc. Of IEEE Int. Conf. on Cybernetics and Society, pp. 163-165, Sept. 1975.

[46] P. A. Viola, Alignment by Maximization of Mutual Information, PhD thesis, Massachusetts Institute of Technology, June 1995.

[47] INRIA, Multimodal Image Registration by Maximization of the Correlation Ratio, vol. 3378, 1998.

[48] H. Kalinic, S. Loncaric and B. Bijnens. "A novel image similarity measure for image registration", 7th International Symposium on Image and Signal Processing and Analysis (ISPA), 2011.

[49] T. Itou, H. Shinohara, K. Sakaguchi, T. Hashimoto, T. Yokoi and T. Souma. "Multimodal image registration using IECC as the similarity measure", Med. Phys. 38(2), 2011.

[50] M. S. Aslan, A. Ali, A. A. Farag, B. Arnold, D. Chen, and P. Xiang. 3D Vertebrae Segmentation in CT Images with Random Noises, Proceedings of the International Conference on Pattern Recognition (ICPR'10), 2010.

[51] J. Yang and D. Zhang and A. F. Frangi and J. Yang. Two-dimensional PCA: A new approach to appearance based face representation and recognition. IEEE Transactions on Pattern Analysis and Machine Intelligence, 26(1), 2004.

[52] M. S. Aslan, A. Ali, D. Chen, B. Arnold, A. A. Farag, and P. Xiang, 3D vertebrae segmentation using graph cuts with shape prior constraints, Proc. of IEEE International Conference on Image Processing (ICIP), 2010.

[53] Y. Kang, K. Engelke, and W. A. Kalender, New accurate and precise 3D segmentation method for skeletal structures in volumetric CT data, IEEE Transaction on Medical Imaging (TMI), 22(5), 2003.

[54] W. A. Kalender, D. Felsenberg, H. Genant, M. Fischer, J. Dequeker, and J. Reeve, The european spine phantom - a tool for standardization and quality control in spinal bone measurements by DXA and QCT.J. Radiology, 20, 1995.

[55] A. Mastmeyer, K. Engelke, C. Fuchs, and W. A. Kalender, A hierarchical 3d segmentation method and the definition of vertebral body coordinate systems for QCT of the lumbar spine, Medical Image Analysis, 10(4), 2006. 
[56] J. Kaminsky, P. Klinge, M. Bokemeyer, W. Luedemann, and M Samii, Specially adapted interactive tools for an improved 3d-segmentation of the spine, Computerized Medical Imaging and Graphics, 28(3), 2004.

[57] M. S. Aslan, A. Ali, H. Rara, B. Arnold, A. A. Farag, R. Fahmi, and P. Xiang, A Novel 3D Segmentation of Vertebral Bones from Volumetric CT Images Using Graph Cuts, ISVC'09, 2009.

[58] M. S. Aslan, H. Abdelmunim, A. A. Farag, B. Arnold, E. Mustafa, and P. Xiang, A new shape based segmentation framework using statistical and variational methods, Proc. of IEEE International Conference on Image Processing (ICIP), 2011.

[59] H. Abdelmunim, A. A. Farag, Curve/Surface Representation and Evolution Using Vector Level Sets with Application to the Shape-Based Pattern Analysis and Machine Intelligence, IEEE Transactions on, 29(6), 2007.

[60] B. Wang, X. Gao, D. Tao, And X. Li, A Unified Tensor Level Set for Image Segmentation, IEEE transactions on systems, man, and cybernetics, Vol. 40(3), June, 2010 .

[61] M. S. Aslan, E. Mostafa, H. Abdelmunim, A. Shalaby, Aly A. Farag, and B. Arnold, A novel probabilistic simultaneous segmentation and registration using level set, Proceedings of the International Conference on Image Processing (ICIP), 2011.

[62] M. S. Aslan, A. Ali, A. A. Farag, H. Abdelmunim, B. Arnold, P. Xiang, A new segmentation and registration approach for vertebral body analysis, Proc. of IEEE International Symposium on Biomedical Imaging (ISBI), 2011.

[63] M. Aslan*, A. Shalaby*, H. Abdelmunim and A. A. Farag, "A Probabilistic Shapebased Segmentation Method Using Level Sets", IET Computer Vision. To Be Published 2013. (* These authors are equally contributing in this work)

[64] T.F. Chen and L.A. Vese, Active contours without edge, IEEE Trans Image Process, Vol. 10(2), pp. 266-277, Feb. 2001.

[65] A. Shalaby, M. Aslan, H. Abdelmunim and A. A. Farag, "2D-PCA based shape prior for level sets segmentation framework for the vertebral body," Proc. of the 6th Cairo International Conference on Biomedical Engineering, (CIBEC'12), Cairo, Egypt, December 22-25, 2012

[66] P. Markelj, D. Tomazevic,B. Likar, F. Pernus, "A review of 3D/2D registration methods for image-guided interventions", Med Image Analysis. April 13, 2010.

[67] P. Bifulco, M. Cesarelli, R. Allen, and et al, "2D-3D Registration of CT Vertebra Volume to Fluoroscopy Projection: A Calibration Model Assessment", Journal on Advances in Signal Processing, 2009.

[68] G.P. Penney, J. Weese, J.A. Little, P. Desmedt, D.L.G. Hill and D.J. Hawkes "A Comparison of Similarity Measures for Use in 2D-3D Medical Image Registration" ,IEEE Transactions on Medical Imaging 17(4), 1998. 
[69] J. Weese, G. P. Penney, P. Desmedt, D.L.G. Hill, and D.J. Hawkes, "Voxel-Based 2D/3-D Registration of Fluoroscopy Images and CT Scans for Image-Guided Surgery", IEEE Transactions on Information Technology in Biomedicine 1(4), Dec. 1997.

[70] J. Weese, T. M. Buzug, C. Lorenz, C. Fassnacht "An Approach to 2D/3D Registration of a Vertebra in 2D X-ray Fluoroscopies with 3D", CVRMed/MRCAS'97, France, March 19-22, 1997.

[71] X. Chen, R. C. Gilkeson, and B.Feia, "Automatic 3D-to-2D registration for CT and dual-energy digital radiography for calcification detection", Med Phys, 34(12), Dec 2007.

[72] L. Zollei, "2D-3D Rigid-Body Registration of X-Ray Fluoroscopy and CT Images", Master Thesis Massachusetts Institute of Technology, 2001.

[73] P. Viola and W. Wells, "Alignment by Maximization of Mutual Information". International Journal of Computer Vision, 241997.

[74] P.W. Pluim, J. B. A. Maintz and M. A. Viergever "Image Registration by Maximization of Combined Mutual Information and Gradient Information", Proceedings of MICCAI 2000.

[75] L. Lemieux, R. Jagoe, D.R. FIsh, N.D. Kitchen, D.G.T. Thomas, "A patient tocomputed-tomography image registration method based on digitally reconstructed radiographs", Med. Phys. 21(11), 1994.

[76] L. M. G. Brown, "Registration of Planar Film Radiographs with Computed Tomography" IEEE; Proceedings of MMBIA 1996.

[77] H. Kalinic, S. Loncaric and B. Bijnens. "A novel image similarity measure for image registration", 7th International Symposium on Image and Signal Processing and Analysis (ISPA), 2011.

[78] J. Maintz and M. Viergever, "A survey of medical image registration," Medical Image Analysis, 2(1), 1998.

[79] http://www.myanklereplacement.com/

[80] T. Itou, H. Shinohara, K. Sakaguchi, T. Hashimoto, T. Yokoi and T. Souma. "Multimodal image registration using IECC as the similarity measure", Med. Phys. 38(2), 2011.

[81] A. Shalaby, A. A. Farag, A. Ross and T. Hockenbury, "2D-3D Registartion of Human Ankle using X-ray and CT images," Proc. of the 6th Cairo International Conference on Biomedical Engineering, (CIBEC'12), Cairo, Egypt, December 22-25, 2012.

[82] A. Shalaby, A. Mahmoud, A. Abdoulmalek, and A. A. Farag, "Registration of Human Foot using X-ray and CT images," Proc. of 15th Saudi Technical Exchange Meeting, (STEM'12), Dhahran, Saudi Arabia, pp. 86 - 89, December 17-19, 2012. 
[83] R. Sandhu, S. Dambreville, A. Yezzi, and A. Tannenbaum. A nonrigid kernelbased framework for $2 \mathrm{~d}-3 \mathrm{~d}$ pose estimation and $2 \mathrm{~d}$ image segmentation. IEEE Transactions on Pattern Analysis and Machine Intelligence, 33(6):1098-1115, 2011.

[84] L. D. Cohen. On active contour models and balloons. CVGIP: Imag.Under., 53(2):211-218, 1991.

[85] V. Caselles, F. Catte, T. Coll, and F. Dibos. A geometric model for active contours. Numerische Mathematik, 66:1-31, 1993.

[86] R. Malladi, J. A. Sethian, and B. C. Vemuri. Shape modeling with front propagation: a level set approach. IEEE T. Patt. Anal. Mach. Intell., 17(2):158-175, 1995.

[87] V. Caselles, R. Kimmel, and G. Sapiro. Geodesic active contours. In Proc. Int'l Conf. Comp. Vis., pages 694-699, 1995.

[88] A. Yezzi, S. Kichenassamy, A. Kumar, P. Olver, and A. Tannenbaum. A geometric snake model for segmentation of medical imagery. IEEE T. Med. Imag., 16:199-209, 1997.

[89] R. Durikovic, K. Kaneda, and H. Yamashita. Dynamic contour: a texture approach and contour operations. The Visual Computer,2011, 277-289, 1995.

[90] C. Xu and J. L. Prince. Snakes, shapes, and gradient vector flow. IEEE T. Imag. Proc., 7(3):359-369, 1998.

[91] V. Caselles, R. Kimmel, and G. Sapiro. Geodesic active contours. Int'l J. Comp. Vis., 22:61-79, 1997.

[92] S. Kichenassamy, A. Kumar, P. Olver, A. Tannenbaum, and A. Yezzi. Conformal curvature flows: from phase transitions to active vision.Arch. Rational Mech. Anal., 134:275-301, 1996.

[93] K. Siddiqi, Y. B. Lauzi 'ere, A. Tannenbaum, and S. W. Zucker. Area and length minimizing flows for shape segmentation. IEEE T. Imag. Proc., 7:433-443, 1998.

[94] N. Paragois and R. Deriche. Unifying boundary and region-based information for geodesic active tracking. In Proc. Comp. Vis. Patt. Recog., pages 300-305, 1999.

[95] N. Paragios and R. Deriche. Geodesic active contours and level sets for the detection and tracking of moving objects. IEEE T. Patt. Anal.Mach. Intell., 22(3):1-15, 2000.

[96] C. Samson, L. Blanc-Feraud, G. Aubert, and J. Zerubia. "A Level Set Method for Image Classification," Int. Conf. Scale-Space Theories in Computer Vision, pp. 306317, 1999.

[97] A. Yezzi, A. Tsai, and A. Willsky, "A Statistical Approach to Image Segmentation for Bimodal and Trimodal Imagery," Proceedings of ICCV, September, 1999.

[98] A. Yezzi, A. Tsai, and A. Willsky, "A Fully Global Approach to Image Segmentation via Coupled Curve Evolution Equations," To appear in J. of Visual Communication and Image Representation. 
[99] C. Xu, D. L. Pham, and J. L. Prince, "Image Segmentation Using Deformable Models," Handbook of Medical Imaging: Vol. 2. Medical Image Processing and Analysis, SPIE Press, 2000.

[100] Grayson, M., The heat equation shrinks embedded plane curves to round points, J. Diff. Geom., Vol. 26, 285, 1987.

[101] J.A .Sethian, Level Set Methods: Evolving Interfaces in Geometry, Fluid Mechanics, Computer Vision and Material Science, Cambridge University Press, 1996.

[102] T. J. Barth and J. A. Sethian, Numerical Schemes for the Hamilton- Jacobi and Level Set Equations on Triangulated Domains, submitted for publication, J. Comp. Physics, Sept., 1997.

[103] G. M. Blake, H. W. Wahner, and I. Fogelman. Dual energy x-ray absorptiometry and ultrasoung in clinical practice. Martin Dunitz, 1999.

[104] S. Tapp. A markov model of secondary prevention of osteoporotic hip fractures. Ph.D. Dissertation, 2003.

[105] Department of Health and Human Services. A report of the surgeon general: Bone Health and Osteoporosis. U. S. Public Health Service, 2004.

[106] M. Roberts, T. Cootes, and J. Adams. Vertebral shape: Automatic measurement with dynamically sequenced active appearance models. In Proceedings of the International Conference on Medical Image Computing and Computer Assisted Intervention, (2), pages 733-740, 2005.

[107] T. Klinder, J. Ostermann, M. Ehm, A. Franz, R. Kneser, C. Lorenz. Automated model-based vertebra detection, identification, and segmentation in CT images. Medical Image Analysis, vol. 13, pp. 471-482, 2009.

[108] S. Tan, J. Yao, M. M. Ward, L. Yao, and R. M. Summers. Computer aided evaluation of ankylosing spondylitis using high-resolution ct. IEEE Transaction on Medical Imaging (TMI), vol. 27, no. 9, pp. 1252-1267, 2008.

[109] J. J. Criscob, B. Sebastiana, H. Teka and B. B. Kimia. Segmentation of carpal bones from ct images using skeletally coupled deformable models. Medical Image Analysis, vol. 7, no. 1, pp. 21-45, 2003.

[110] Y. Kim, and D. Kim. A fully automatic vertebra segmentation method using 3d deformable fences. Computerized Medical Imaging and Graphics, vol. 33, pp. 343$352,2009$.

[111] M. Leventon,R., W. Eric L. Grimson, and O. Faugeras. Statistical shape influence in geodesic active contours. In Proce. IEEE CVPR, pp.1316-1324, 2000.

[112] Y. Chen, S. Thiruvenkadam, F. Huang, D. Wilson, E. A. Geiser, and H. D. Tagare. On the incorporation of shape priors into geometric active contours. In IEEE VLSM, pp. 145-152, 2001. 
[113] M. S. Aslan, A. Ali, H. Rara, and A. A. Farag, "An Automated Vertebra Identification and Segmentation in CT Images," Proc. of 2010 IEEE International Conference on Image Processing (ICIP), pp. 233-236, 2010.

[114] P. H. Lim, U. Bagci, O. Aras, Y. Wang, and L. Bai, "A novel spinal vertebrae segmentation framework combining geometric flow and shape prior with level set method," in Proc. IEEE Int. Symp. Biomed. Imag.Barcelona, Spain, May 2012, pp. $1703-1706$.

[115] D. Cremers, S. J. Osher, and S. Soatto, "Kernel density estimation and intrinsic alignment for shape priors in level set segmentation," Int. J. Comput. Vis., vol. 69, no. 3, pp. 335-351, 2006.

[116] R. Schneider and L. Kobbelt, "Generating fair meshes with G1 boundary conditions," in Proc. Geom. Model. Process. Conf., 2000, pp. 251-261.

[117] S. Yoshizawa and A. G. Belyaev, "Fair triangle mesh generation withdiscrete elastica," in Proc. Geom. Model. Process. 2002, pp. 119-123.

[118] J. W. Barrett, H. Garcke, and R. Nrnberg. (2008, Jan.). Parametric approximation of willmore flow and related geometric evolution equations. SIAM J. Sci. Comput. [Online]. 31(1), pp. 225-253.

[119] T. J. Willmore, "Note on embedded surfaces," Analele S, tiint, ifice ale Universit at, ii Al. I. Cuza din Ias, i. Serie Nou?a, vol. Ia 11B, pp. 493-496, 1965.

[120] M. Droske and M. Rumpf. (2004). A level set formulation for Willmore flow. Interfac. Free Boundar. [Online]. 6(3), pp. 361-378.

[121] L. Dice, "Measures of the amount of ecologic association between species," Ecology, vol. 26, pp. 297-302, 1945.

[122] R. T. Rockafellar and R. J.-B. Wets, Variational Analysis. NewYork: SpringerVerlag, 2005.

[123] C. Li, C. Xu, C. Gui, M. D. Fox,"Level set evolution without re-initialization: A new variational formulation," Proc. of the 2005 IEEE Computer Society Conference on Computer Vision and Pattern Recognition (CVPR'05), 2005.

[124] S. H. Zhou, I. D. McCarthy, A. H. McGregor, R. R. H. Coombs, and S. P. F. Hughes. (2000, Jun.). Geometrical dimensions of the lower lumbar Vertebrae analysis of data from digitized CT images. Eur. Spine J. [Online]. 9(3), pp. 242-248. 


\section{CURRICULUM VITAE}

\section{Full Name:}

\section{Ahmed Magdy Ahmed Shalaby}

\section{Personal Information}

Marital status: Married (with Three Children).

Nationality: Egyptian.

Date of Birth: May 22, 1982.

Place of Birth: Alexandria, Egypt.

Home Address: 788 Raymond Kent Ct, Apt 4

Louisville, KY

40217, USA

Phone: +1 (502) 821-0974

E-mail: ahmed.shalaby@louisville.edu

Homepage: http://www.cvip.louisville.edu/shalaby.htm

\section{Education}

Ph.D., University of Louisville, KY, USA, Fall 2014

-Thesis: Registration Techniques for Medical Imaging: Novel Algorithms and Applications.

- Supervisor: Prof. Aly A. Farag.

- GPA: 3.975.

M.Sc., Alexandria University, Alexandria, Egypt, 2009

- Thesis: Multiuser detection for Optimum MC-CDMA Communication Systems.

- Supervisors: Prof. Said Elnoubi, Dr. Adel Elfahar.

-GPA: 4.0.

B.Sc., Alexandria University, Alexandria, Egypt, 2002

- Cumulative Grade: Distinction with Degree of Honor (89.25\%).

- Department Rank: Third over a class of 350 students.

- Graduation Project: Bluetooth ${ }^{\mathrm{TM}}$ System \& Applications.

\section{Professional and Work Experience}

University of Louisville: 2011 - 2012 (Part Time)

- Stochastic Process (ECE 530).

- Pattern Recognition (ECE 620). 
Arab Academy for Science \& Technology, Alexandria, Egypt: 2007 - 2009 (Part Time)

- Communication Systems (1): Analog communications.

- Communications Systems (2): Random signals \& noise analysis.

- Electrical circuits Analysis.

- Electronic Measurements.

Alexandria University, Alexandria, Egypt: 2004 - 2009 (Full Time)

- Teaching: Taught courses on the following subjects:

1. Signal and Systems

2. Communications Systems (1)

3. Communications Systems (2)

4. Fundamental of Electrical Circuits

5. Analysis of Electrical Circuits

6. Logic Design

7. Analog filters design

- Labs:

1. Fundamental of Electrical Circuits

2. Analog Communications systems

3. Digital Communications systems

4. Electronic Measurements

5. Electronic Circuits Analysis

6. Logic Design

7. Acoustics

Egyptian Engineers Company, summer 2002 Internship, Cairo, Egypt.

Alexandria Petroleum Company, summer 2000 Internship, Alexandria, Egypt.

Arabia Computer Systems (ACS): Summer 1999 Internship, Alexandria, Egypt.

\section{Training Courses and Certificates}

University Legal and Financial Aspects, Faculty and Leadership Development Center (FLDC), Alexandria University, December 2009.

Competitive Research Projects, Faculty and Leadership Development Center (FLDC), Alexandria University, September 2009.

Effective Presentation Skills, Faculty and Leadership Development Center (FLDC), Alexandria University, September 2009.

International Publishing of Research, Faculty and Leadership Development Center (FLDC), Alexandria University, September 2008.

References Management Systems, Faculty and Leadership Development Center (FLDC), Alexandria University, September 2008.

Student Evaluation and Examination Techniques, Faculty and Leadership Development Center (FLDC), Alexandria University, August 2008. 


\section{Book Chapters}

1. A. Shalaby, A. A. Farag, E. Mostafa and T. Hockenbury, "2D/3D Registration: A Step Towards Image-Guided Ankle Fusion" in: BioImaging and Visualization for Patient-Customized Simulations. Springer, series: LNCVB, Vol. 13, p. 137, 2014.

2. A. Shalaby, M. Aslan, and A. A. Farag, "A Probabilistic Shape- Based Segmentation method of VBs using Level Sets" in: Computational Methods and Clinical Applications for Spine Imaging. Springer, series: LNCVB, 2014.

3. A. Farag, A. Shalaby, H. Abd El Munim and A. A. Farag, "Variational Shape Representation for Modeling, Elastic Registration and Segmentation" in: Shape Analysis in Medical Image Analysis. Springer, series: LNCVB, Vol. 14, p. 442, 2014.

4. M. Aslan, A. Shalaby, and A. A. Farag, "Clinically Desired Segmentation Method for Vertebral Bodies" in: Computational Methods and Clinical Applications for Spine Imaging. Springer, series: LNCVB, 2014.

\section{Journal Publications}

5. A. Shalaby, M. Aslan, and A. A. Farag, "3D Simultaneous Segmentation and Registration of Vertebral Bodies for accurate BMD measurement", IEEE TBME-2014 under review.

6. A. Shalaby, M. Aslan, and A. A. Farag, "Vertebral Body Segmentation Using a Probabilistic and Universal Shape Model", IET Computer Vision accepted to appear in 2014.

7. M. Aslan*, A. Shalaby*, H. Abdelmunim and A. A. Farag, "A Probabilistic Shape-based Segmentation Method Using Level Sets", IET Computer Vision, 2013, DOI: 10.1049/ietcvi.2012.0226.

\section{Conference Publications}

8. El-Barkouky, A. Shalaby, A. Mahmoud, A .A. Farag, Selective Part Models for Detecting Partially Occluded Faces in the Wild, The IEEE International Conference on Image Processing, ICIP 2014, Oct 27-30, 2014, Paris, France.

9. Shalaby, M. Aslan, E. Mostafa and A .A. Farag, 2D-PCA based Tensor Level Set Framework for Vertebral Body Segmentation, MICCAI 2013 workshop on Computational Methods and Clinical Applications for Spine Imaging, Sept 22-26, 2013, Tokyo, Japan.

10. Shalaby, E. Mostafa, T. Hockenbury and A. A. Farag, 2D-3D Registration: A Step Towards ImageGuided Ankle Fusion, MICCAI 2013 workshop on Bio- Imaging and Visualization for PatientCustomized Simulations, Sept 22-26,2013, Tokyo, Japan.

11. M. Aslan*, A. Shalaby*, and A.A. Farag, "Clinically Desired Segmentation Method for Vertebral Bodies," Proc. of 2013 International Symposium on Biomedical Imaging (ISBI'13), San Francisco, USA, April 7-11, 2013. 
12. A . Abdelrahim*, A. Shalaby*, S. Elhabian, J. Graham, and A. Farag," A 3d Reconstruction Of The Human Jaw From A Single Image", Proc. of 2013 IEEE International Conference on Image Processing (ICIP), accepted to appear.

13. A. Shalaby, A. A. Farag, A. Ross and T. Hockenbury, "2D-3D Registartion of Human Ankle using X-ray and CT images," Proc. of the 6th Cairo International Conference on Biomedical Engineering, sponsored by the IEEE Engineering in Medicine and Biology Society, (CIBEC'12), Cairo, Egypt, December 22-25, 2012.

14. A.Shalaby, M. Aslan, H. Abdelmunim and A. A. Farag, "2D-PCA based shape prior for level sets segmentation framework for the vertebral body," Proc. of the 6th Cairo International Conference on Biomedical Engineering, sponsored by the IEEE Engineering in Medicine and Biology Society, (CIBEC'12), Cairo, Egypt, December 22-25, 2012.

15. A. Shalaby, A. Mahmoud, E. Mostafa, A. Abdoulmalek, and A. A. Farag, " Segmentation framework of vertebral body using 2D-PCA," Proc. of 15th Saudi Technical Exchange Meeting, (STEM'12), Dhahran, Saudi Arabia, pp. 81 - 85, December 17-19, 2012.

16. A. Shalaby, A. Mahmoud, A. Abdoulmalek, and A. A. Farag, "Registration of Human Foot using X-ray and CT images," Proc. Of $15^{\text {th }}$ Saudi Technical Exchange Meeting, (STEM'12), Dhahran, Saudi Arabia, pp. 86 - 89, December 17-19, 2012.

17. M. S. Aslan, E. Mustafa,H. Abdelmunim, A. Shalaby, Aly A. Farag, and Ben Arnold, "A novel probabilistic simultaneous segmentation and registration using level set," Proc. Of 2011 IEEE International Conference on Image Processing (ICIP), pp. 2161 - 2164, Sept 2011.

18. A. Shalaby, A. Ali, A. A. Farag, "Simultaneous Identification and Tracking of Moving Targets," Proc. of $8^{\text {th }}$ IEEE Workshop on Object Tracking and Classification Beyond the Visible Spectrum (OTCBVS), pp. 49 - 54, June 2011.

19. A. Shalaby, S. El-kashlan, "Mixing Wavelet Transform and Self-Organizing Mapping Network for Mitigating Load Capacitor Switching “, MEPCON'09, 2009.

20. A. Shalaby, S. Elnoubi, and A. Elfahar," Ant colony approach for optimum multiuser detector of MC-CDMA system", NRSC, 2008.

\section{Awards Received}

Electrical Engineering Outstanding Graduate Student Award, University of Louisville, 2013

Theobald Scholarship Award, University of Louisville, 2013

Graduate Student Scholarship, University of Louisville, Fall 2011-Spring 2014.

Degree of Honor of Cumulative Academic Distinction, Alexandria University, June 2003

Academic Distinction Award, Faculty of Engineering, Alexandria University, 2000 - 2003.

* These authors are equally contributing to this work 


\section{Language and Computer Skills}

Languages: Arabic (Native), English (Very good).

Computer Skills: Programming (Matlab, Visual C++, C\#), Microsoft Office, Windows, Linux.

\section{Interests and Activities}

Communication \& Electronics, Computer Hardware, Reading, Traveling and social relationships

\section{References}

Available upon request 